ITP-SB-96-40

\title{
Covariant Computation of the Low Energy Effective Action of the Heterotic Superstring
}

\author{
Jan de Boer[] And Kostas Skenderis], \\ Institute for Theoretical Physics \\ State University of New York at Stony Brook \\ Stony Brook, NY 11794-3840, USA
}

\begin{abstract}
We derive the low energy effective action of the heterotic superstring in superspace. This is achieved by coupling the covariantly quantized Green-Schwarz superstring of Berkovits to a curved background and requiring that the sigma model has superconformal invariance at tree level and at one loop in $\alpha^{\prime}$. Tree level superconformal invariance yields the complete supergravity algebra, and one-loop superconformal invariance the equations of motion of the low energy theory. The resulting low energy theory is old-minimal supergravity coupled to a tensor multiplet. The dilaton is part of the compensator multiplet.
\end{abstract}

\footnotetext{
${ }^{1}$ e-mail: deboer@insti.physics.sunysb.edu, address after September 1st: Department of Physics, University of California at Berkeley, 366 LeConte Hall, Berkeley, CA 94720-7300

${ }^{2}$ e-mail: kostas@insti.physics.sunysb.edu, address after September 1st: Instituut voor Theoretische Fysica, Katholieke Universiteit Leuven, Celestijnenlaan 200D, B-3001 Leuven, Belgium
} 


\section{Introduction}

Low energy effective actions play an important role in the study of string theory, and not only for phenomenological applications. They also provide important pieces of evidence for the existence of various dual descriptions of string theories. One can consider low energy effective actions in their own right as specific examples of locally supersymmetric field theories. The structure of these low energy effective field theories is constrained not only by the requirement of local supersymmetry, but also by the fact that they represent low energy effective theories of some string theory. It is clearly desirable to obtain as much understanding of these constraints as possible. One of the most powerful ways to obtain such constraints is by analyzing the two-dimensional sigma model that describes the string theory coupled to the background fields of the low energy effective field theory. Unfortunately, until recently no sigma model description was known which was formulated directly in terms of a target superspace and which had manifest local target space supersymmetry (this problem is also known as 'the covariant quantization of the superstring'). Without such a sigma model description, one can only work in components and not determine the off-shell description that is selected by string theory. In addition, it is in general quite difficult to obtain the fermionic part of the effective action without a manifestly supersymmetric sigma model. In the RNS formalism for example, the fermionic terms can only be obtained by a calculation of S-matrix scattering amplitudes.

It is important to know the precise off-shell description, as it provides constraints on the allowed types of matter couplings in the low energy effective action. Furthermore, it provides constraints on the structure of higher order $\alpha^{\prime}$ corrections, and it provides a natural framework to study the interplay between $T$-duality and supersymmetry, supersymmetry breaking, and non-renormalization theorems.

The sought for sigma model description of string theory was obtained recently by Berkovits [1, 2, 3]. In the case of heterotic string theory compactified to four dimensions, it is a sigma model with $N=(2,0)$ supersymmetry on the world-sheet. The coupling of the theory to background fields was discussed in [4], where also the form of the lowenergy effective action was proposed, based on indirect arguments. In this paper we perform a detailed study of this sigma model. We show how the tree-level analysis of the sigma model yields a complete set of supergravity constraints, and how this determines, by means of the Bianchi identities, the supergravity algebra. Next, we study the sigma model at one loop, and find the equations of motion of the supergravity theory, all in superspace. We determine the action that reproduces these field equations, and thereby establish that the theory that describes the four dimensional fields of the compactification of the heterotic string is old-minimal supergravity coupled to a tensor multiplet. The dilaton is part of the chiral and anti-chiral compensator fields [5, 6], and not, as is often claimed, part of the tensor multiplet. This affects, in the presence of charged matter fields, the structure of the effective action, especially at higher string loops.

The outline of this paper is as follows. In section two we review the new covariant sigma model description for the heterotic string. We first describe the sigma model in flat space, in which case it is an exact conformal field theory. Next we couple the sigma model to a curved background. To do this we first rewrite the action in the flat background in a manifestly supersymmetric way, and then promote the flat vielbeins and antisymmetric 
tensor fields into arbitrary unconstrained fields. The sigma model is then by construction target space super-reparametrization invariant. Moreover, when expanded around a flat background it contains all massless vertex operators of the heterotic superstring. For simplicity we set both the compactification dependent and the Yang-Mills background fields equal to zero. Our method can easily be extended to include these fields as well. Next, we add a Fradkin-Tseytlin term to the sigma model to accomodate the dilaton. The structure of the Fradkin-Tseytlin term, proposed in [4], forces the dilaton to be part of a chiral and anti-chiral target space superfield, and shows that it cannot sit in the same multiplet as the antisymmetric tensor field.

The sigma-model, thus constructed, has a local target space Lorentz and $U(1)$ invariance. In section 3 we show how to maintain this symmetry at the quantum level using the background field method. We develop a background field expansion that it is covariant with respect to both the Lorentz and the $U(1)$ symmetry. One of the interesting features of the sigma model is that it has a chiral boson $\rho$ whose kinetic term is $\alpha^{\prime}$ independent, and which has to be treated exactly. This can be achieved by means of a field redefinition, and allows us to develop a hybrid perturbation theory where we treat all fields perturbatively except the field $\rho$ that we treat exactly. We use this perturbation theory to analyze the sigma model at tree level. Due to the presence of the chiral boson $\rho$, a Poisson bracket analysis of the theory would not be sufficient, and one has to analyze tree diagrams in perturbation theory instead. From the tree diagrams we obtain a set of constraints, which, when supplemented with a maximal set of conventional constraints, provides a complete set of constraints for conformal supergravity coupled to a tensor multiplet. This, as well as the complete supergravity algebra, follows from the solution to the Bianchi identities.

In section 4 , we check the superconformal invariance of the sigma model at one loop, by computing the OPE's of the $N=2$ generators in perturbation theory. We evaluate all one-loop graphs in momentum space using a version of dimensional regularization suitable for this sigma model. We find that the non-holomorphic terms in the OPE's yield the field equations of the low energy effective field theory. The extra holomorphic terms that appear in the calculation but should not be there can all be removed using suitable one-loop counterterms and a modification of the background field expansion. The latter is, unfortunately, rather ad hoc, and we have not yet understood whether this has a geometrical interpretation or is an artifact of the scheme we use. We conclude this section by a discussion of sigma model anomalies, and show how they can be cancelled by a superspace version of the Green-Schwarz mechanism. Also, we briefly indicate which part of the calculation is reproduced by a standard beta-function calculation. Such a calculation would be the most efficient way to obtain some results at two loops.

Having obtained the field equations we then look in section 5 for a supergravity action that reproduces the same field equations. This is a rather tedious exersice in supergravity theory. Although it is straightforward to write down candidate actions, to find out what their field equations are is not an easy task. This is because the supergravity fields are constrained. Hence, when varying the action to obtain the field equations the variations should respect the constraints. To achieve this we express the variations in terms of unconstrained prepotentials. Our final result is that the low energy effective theory of the heterotic superstring is old-minimal supergravity coupled to a tensor multiplet.

In section six we present our conclusions and some possible applications of our results. 
The superspace conventions we used throughout the paper are summarized in appendix A, and appendix B provides details of the solution of the Bianchi identities presented in the main text.

\section{The covariant description of $4 \mathrm{~d}$ compactifications of the heterotic string}

\section{$2.1 \quad$ Flat space}

In this section we review the construction and some of the properties of the two-dimensional sigma model which is manifestly space-time supersymmetric and describes $4 \mathrm{~d}$ compactifications of the heterotic string. This covariant description originated in [1] and was further developed in [2], [3] and [4]. For a review, see [7].

An important distinction between the covariant formulation and the RNS or GreenSchwarz formulations of the heterotic string is that in the covariant formalism the heterotic string arises as a critical $(2,0)$ string theory rather than a $(1,0)$ one. In a flat $4 \mathrm{~d}$ background, the $4 \mathrm{~d}$-part of the heterotic string is in the $(2,0)$ superconformal gauge desribed by the following action ${ }^{3}$

$$
S=\frac{1}{\alpha^{\prime}} \int d^{2} z\left(\frac{1}{2} \partial x^{m} \bar{\partial} x^{m}+p_{\alpha} \bar{\partial} \theta^{\alpha}+p_{\dot{\alpha}} \bar{\partial} \bar{\theta}^{\dot{\alpha}}-\frac{\alpha^{\prime}}{2} \bar{\partial} \rho\left(\partial \rho+a_{z}\right)\right)
$$

where $z^{M} \equiv\left(x^{m}, \theta^{\alpha}, \bar{\theta}^{\dot{\alpha}}\right)$ are the coordinates of flat $4 \mathrm{~d}$ superspace, the $p$ 's are the conjugate fields of the $\theta$ 's and $\rho$ is a boson with a 'wrong' sign for the kinetic term. We omitted here the $32-2 r$ heterotic fermions and the $c=(9,6+r)$ internal superconformal field theory coming from the Calabi-Yau sector, as we will not consider background fields coming from these sectors in this paper. However, our methods can be easily extended to include these fields as well. Notice the explicit factor of $\alpha^{\prime}$ in front of $\rho$, from which it follows that the $\rho$-sector of the theory is completely $\alpha^{\prime}$-independent. One of the consequences this will have for us later is that in order to obtain results at a fixed order in $\alpha^{\prime}$, we need to treat the $\rho$-sector exactly rather than perturbatively. The $U(1)$ gauge field component $a_{z}$ will be viewed as a Lagrange multiplier imposing the constraint $\bar{\partial} \rho=0$, so that $\rho$ becomes a chiral boson. If we couple the theory to $(2,0)$ world-sheet supergravity, the theory will contain both components $a_{z}$ and $a_{\bar{z}}$ of a $U(1)$ gauge field. One might think that the gauge fixed theory should therefore have both a left and right moving $U(1)$ symmetry, but this is not quite true, since the two $U(1)$ gauge fields gauge only one symmetry ( $\rho \rightarrow \rho+$ constant), so that only one component of the gauge field can be gauged away. The other remains as a Lagrange multiplier (modulo global issues, which we ignore in the remainder).

From the action (2.1) one derives the following OPE's

$$
\partial x_{\alpha \dot{\alpha}}(z) \partial x_{\beta \dot{\beta}}(w)=\frac{-\alpha^{\prime} C_{\alpha \beta} C_{\dot{\alpha} \dot{\beta}}}{(z-w)^{2}}
$$

\footnotetext{
${ }^{3}$ We use an Euclidean world-sheet, and the measure $d^{2} z$ denotes $d x d y / \pi$, with $z=x+i y$. The advantage of this convention is that we never see explicit factor of $\pi$. In particular the following ditributional identity holds: $\bar{\partial}_{\bar{z}}(z-w)^{-1}=\delta^{(2)}(z-w)$, where $\delta^{(2)}(z-w)$ is the delta function with respect to the measure $d^{2} z$.
} 


$$
\begin{aligned}
p_{\alpha}(z) \theta^{\beta}(w) & =\frac{\alpha^{\prime} \delta_{\alpha}{ }^{\beta}}{(z-w)} \\
p_{\dot{\alpha}}(z) \bar{\theta}^{\dot{\beta}}(w) & =\frac{\alpha^{\prime} \delta_{\dot{\alpha}}^{\dot{\beta}}}{(z-w)} \\
\partial \rho(z) \partial \rho(w) & =\frac{1}{(z-w)^{2}}
\end{aligned}
$$

It has a $(2,0)$ superconformal symmetry on the world-sheet with generators

$$
\begin{aligned}
T & =\frac{1}{\alpha^{\prime}}\left(-\frac{1}{2} \partial x^{\alpha \dot{\alpha}} \partial x_{\alpha \dot{\alpha}}-p_{\alpha} \partial \theta^{\alpha}-p_{\dot{\alpha}} \partial \bar{\theta}^{\dot{\alpha}}+\frac{\alpha^{\prime}}{2} \partial \rho \partial \rho\right) \\
G & =\frac{1}{i \alpha^{\prime} \sqrt{8 \alpha^{\prime}}} e^{i \rho} d^{\alpha} d_{\alpha} \\
\bar{G} & =\frac{1}{i \alpha^{\prime} \sqrt{8 \alpha^{\prime}}} e^{-i \rho} d^{\dot{\alpha}} d_{\dot{\alpha}} \\
J & =-i \partial \rho
\end{aligned}
$$

where we defined

$$
\begin{aligned}
& d_{\alpha}=p_{\alpha}+i \bar{\theta}^{\dot{\alpha}} \partial x_{\alpha \dot{\alpha}}+\frac{1}{2} \bar{\theta}^{2} \partial \theta_{\alpha}-\frac{1}{4} \theta_{\alpha} \partial\left(\bar{\theta}^{2}\right) \\
& d_{\dot{\alpha}}=p_{\dot{\alpha}}+i \bar{\theta}^{\alpha} \partial x_{\alpha \dot{\alpha}}+\frac{1}{2} \theta^{2} \partial \bar{\theta}_{\dot{\alpha}}-\frac{1}{4} \bar{\theta}_{\dot{\alpha}} \partial\left(\theta^{2}\right)
\end{aligned}
$$

The generators in (2.3) satisfy the usual $N=2$ superconformal algebra

$$
\begin{aligned}
T(z) T(w) & =\frac{c / 2}{(z-w)^{4}}+\frac{2 T(w)}{(z-w)^{2}}+\frac{\partial T(w)}{(z-w)} \\
T(z) G(w) & =\frac{\frac{3}{2} G(w)}{(z-w)^{2}}+\frac{\partial G(w)}{(z-w)} \\
T(z) \bar{G}(w) & =\frac{\frac{3}{2} \bar{G}(w)}{(z-w)^{2}}+\frac{\partial \bar{G}(w)}{(z-w)} \\
J(z) G(w) & =\frac{G(w)}{(z-w)} \\
J(z) \bar{G}(w) & =\frac{-\bar{G}(w)}{(z-w)} \\
J(z) J(w) & =\frac{c / 3}{(z-w)^{2}} \\
G(z) \bar{G}(w) & =\frac{2 c / 3}{(z-w)^{3}}+\frac{2 J(w)}{(z-w)^{2}}+\frac{2 T(w)+\partial J(w)}{(z-w)}
\end{aligned}
$$

with $c=-3$. This central charge is exactly what we need, since combined with the $c=+9 N=2$ algebra in the Calabi-Yau sector the total $N=2$ algebra has central

\footnotetext{
${ }^{4}$ For simplicity, we will abbreviate $d^{\dot{\alpha}} d_{\dot{\alpha}}$ and $\nabla^{\dot{\alpha}} \nabla_{\dot{\alpha}}$ by $\bar{d}^{2}$ and $\bar{\nabla}^{2}$.
} 
charge $c=+6$, which is precisely the central charge needed to obtain a critical $N=2$ string $(6=26-11-11+2)$. In contrast to the RNS string where only an $N=1$ subalgebra of the $N=2$ algebra of the internal Calabi-Yau sector appears as a local symmetry algebra, here we need the full $N=2$ algebra.

In verifying (2.5) one has to be a bit careful. For example, to verify that $G$ has a regular OPE with itself, one needs to check that the normal ordered product of $(d)^{2}$ with itself vanishes. If this would be nonzero, it would (due to the factor $e^{i \rho}$ in $G$ ) appear as a first order pole in the OPE of $G$ with $G$. The easiest way to verify (2.5) is to use the following intermediate results

$$
\begin{aligned}
d_{\alpha}(z) d_{\beta}(w) & =\text { regular } \\
d_{\dot{\alpha}}(z) d_{\dot{\beta}}(w) & =\text { regular } \\
d_{\alpha}(z) d_{\dot{\beta}}(w) & =\frac{2 i \alpha^{\prime} \Pi_{\alpha \dot{\beta}}}{(z-w)} \\
d_{\alpha}(z) \Pi_{\beta \dot{\beta}}(w) & =\frac{-2 \alpha^{\prime} i C_{\alpha \beta} \partial \bar{\theta}_{\dot{\beta}}}{(z-w)} \\
d_{\dot{\alpha}}(z) \Pi_{\beta \dot{\beta}}(w) & =\frac{-2 \alpha^{\prime} i C_{\dot{\alpha} \dot{\beta}} \partial \theta_{\beta}}{(z-w)} \\
d^{2}(z) d_{\dot{\alpha}}(w) & =-\frac{8\left(\alpha^{\prime}\right)^{2} \partial \bar{\theta}_{\dot{\alpha}}(w)}{(z-w)^{2}}+\frac{4 \alpha^{\prime} i d^{\alpha} \Pi_{\alpha \dot{\alpha}}(w)}{(z-w)} \\
\bar{d}^{2}(z) d_{\alpha}(w) & =-\frac{8\left(\alpha^{\prime}\right)^{2} \partial \theta_{\alpha}(w)}{(z-w)^{2}}+\frac{4 \alpha^{\prime} i d^{\dot{\alpha}} \Pi_{\alpha \dot{\alpha}}(w)}{(z-w)}
\end{aligned}
$$

where $d^{2}=d^{\alpha} d_{\alpha}, \bar{d}^{2}=d^{\dot{\alpha}} d_{\dot{\alpha}}$ and we defined

$$
\Pi_{\alpha \dot{\alpha}}=\partial x_{\alpha \dot{\alpha}}-i \theta_{\alpha} \partial \bar{\theta}_{\dot{\beta}}+i \partial \theta_{\alpha} \bar{\theta}_{\dot{\beta}}
$$

in terms of which $T$ becomes

$$
\frac{1}{\alpha^{\prime}}\left(-\frac{1}{2} \Pi^{\alpha \dot{\alpha}} \Pi_{\alpha \dot{\alpha}}-d_{\alpha} \partial \theta^{\alpha}-d_{\dot{\alpha}} \partial \bar{\theta}^{\dot{\alpha}}+\frac{\alpha^{\prime}}{2} \partial \rho \partial \rho\right)
$$

The advantage of the variables $d$ and $\Pi$ over $p$ and $x$ is that they commute with the target space supersymmetry generators

$$
\begin{aligned}
q_{\alpha} & =\oint \frac{d z}{2 \pi i}\left(p_{\alpha}-i \bar{\theta}^{\dot{\alpha}} \partial x_{\alpha \dot{\alpha}}+\frac{1}{4} \theta_{\alpha} \partial\left(\bar{\theta}^{2}\right)\right) \\
q_{\dot{\alpha}} & =\oint \frac{d z}{2 \pi i}\left(\bar{p}_{\dot{\alpha}}-i \theta^{\alpha} \partial x_{\alpha \dot{\alpha}}+\frac{1}{4} \bar{\theta}_{\dot{\alpha}} \partial\left(\theta^{2}\right)\right)
\end{aligned}
$$

In general, we can define variables $\Pi^{A}, \bar{\Pi}^{A}$ using vielbeins $E_{M^{A}}$ to convert curved into flat indices

$$
\Pi^{A}=\partial z^{M} E_{M}^{A}, \quad \bar{\Pi}^{A}=\bar{\partial} z^{M} E_{M}^{A} .
$$

$\Pi^{\alpha \dot{\alpha}}$ reduces to (2.7) when $E_{M}{ }^{A}$ is the vielbein of flat superspace, which is one on the diagonal and has off-diagonal components

$$
E_{\dot{\mu}}^{\alpha \dot{\alpha}}=i \delta_{\dot{\mu}}^{\dot{\alpha}} \theta^{\alpha}, \quad E_{\mu}^{\alpha \dot{\alpha}}=i \delta_{\mu}^{\alpha} \bar{\theta}^{\dot{\alpha}} .
$$


The action also becomes manifestly target space supersymmetric when written in terms of $\Pi^{A}, \bar{\Pi}^{A}$,

$$
S=\frac{1}{\alpha^{\prime}} \int d^{2} z\left(\frac{1}{2} \Pi^{\alpha \dot{\alpha}} \bar{\Pi}_{\alpha \dot{\alpha}}+d_{\alpha} \bar{\Pi}^{\alpha}+d_{\dot{\alpha}} \bar{\Pi}^{\dot{\alpha}}+\frac{1}{2} \bar{\Pi}^{A} \Pi^{B} B_{B A}-\frac{\alpha^{\prime}}{2} \bar{\partial} \rho\left(\partial \rho+a_{z}\right)\right)
$$

where we introduced an anti-symmetric tensor field $B_{B A}$ whose only non-zero components are

$$
\begin{gathered}
B_{\alpha \dot{\alpha}, \dot{\beta}}=i C_{\dot{\beta} \dot{\alpha}} \theta_{\alpha} \quad B_{\alpha \dot{\alpha}, \beta}=i C_{\beta \alpha} \bar{\theta}_{\dot{\alpha}} \\
B_{\dot{\beta}, \alpha \dot{\alpha}}=-i C_{\dot{\beta} \dot{\alpha}} \theta_{\alpha}
\end{gathered}
$$

Covariant derivatives are in flat superspace given by $\nabla_{A}=E_{A}{ }^{M} \partial_{M}$, where the inverse vielbein $E_{A}{ }^{M}$ has one on the diagonal and off-diagonal components

$$
E_{\dot{\alpha}}{ }^{\mu \dot{\mu}}=-i \delta_{\dot{\alpha}}{ }^{\dot{\mu}} \theta^{\mu}, \quad E_{\alpha}^{\mu \dot{\mu}}=-i \delta_{\alpha}{ }^{\mu} \bar{\theta}^{\dot{\mu}},
$$

as follows from (2.11). From this we deduce that the non-zero torsion of flat superspace is in our conventions given by

$$
T_{\alpha, \dot{\alpha}}^{\beta \dot{\beta}}=-2 i \delta_{\alpha}^{\beta} \delta_{\dot{\alpha}}^{\dot{\beta}}
$$

Associated to the anti-symmetric tensor field in (2.13) is a non-zero field strength $H_{A B C}$. With curved indices it is given by $H_{M N P}=\frac{1}{4} \partial_{[M} B_{N P)}$ which in terms of flat indices becomes

$$
H_{A B C}=\frac{1}{4} \nabla_{[A} B_{B C)}-\frac{1}{4} T_{[A B}^{D} B_{|D| C)} .
$$

The only non-zero components of $H$ are

$$
H_{\alpha, \dot{\alpha}, \beta \dot{\beta}}=-i C_{\alpha \beta} C_{\dot{\alpha} \dot{\beta}}
$$

and its permutations.

\subsection{Relation with RNS and Green-Schwarz formalism}

For completeness, we briefly indicate the relation between this formalism and the usual RNS and Green-Schwarz formalisms[3].

The usual light-cone variables of the four-dimensional Green-Schwarz superstring appear after bosonizing a pair $\left(p_{\alpha}, \theta^{\alpha}\right)$ for $\alpha=1$ or $\alpha=2$, or a pair $\left(p_{\dot{\alpha}}, \bar{\theta}_{\dot{\alpha}}\right)$, and by imposing the constraints that follow from the $N=2$ algebra. The stress-energy tensor $T$ can be used to gauge $\partial\left(x^{0}+x^{3}\right)$ to 1 , and the constraint $T=0$ can be used to eliminate $x^{0}-x^{3}$. The $U(1)$ current $J$ can be used to gauge $\rho=i \sigma$, where $\sigma$ is the boson used to bosonize a pair $(p, \theta)$ via $p=e^{-i \sigma}, \theta=e^{i \sigma}$, and subsequently $J=0$ is used to take $\partial \rho=0$. Next, $G$ and $\bar{G}$ can be used to gauge a $\theta$ and a $\bar{\theta}$ equal to zero, and $G=\bar{G}=0$ can be used to eliminate the two corresponding $p$ 's, so that all that remains is $x^{1}, x^{2}$ and one pair $(p, \theta)$, which are the light-cone Green-Schwarz variables.

The relation with the RNS formalism starts by embedding the $N=1$ RNS string in a critical $N=2$ string following [8]. The cohomologies are the same in the two cases, which can easily be seen from the fact that the two BRST operators are related by a similarity transformation [9]. Subsequently, one needs to perform a field redefinition and a further 
unitary transformation to arrive at the $N=2$ string described in the previous paragraph. A nice check on this procedure is that only those fields in the RNS string that survive the GSO projection can be transformed into a single valued field in the $N=2$ string, which is what one would expect in view of the manifest target space supersymmetry of the latter.

\subsection{The $\sigma$-model in a curved background}

Our next task is to formulate the sigma model in (2.1) in a curved background [4]. The main requirements this formalism should be subject to are: (i) it should be manifestly world-sheet covariant, (ii) it should be target space supersymmetric, (iii) when expanded to first order around a flat background one should recover the massless vertex operators of a flat background and (iv) the complete set of massless physical states of the heterotic superstring should be present.

The massless vertex operators were discussed in [3] and can be obtained, for example, by transforming the vertex operators in the RNS formalism to ones for the $N=2$ string discussed here, as explained in the previous section. They have the form

$$
V=\int d^{2} z\left\{\bar{G},\left[G, V_{\alpha \dot{\alpha}}\right]\right\} \bar{\Pi}^{\alpha \dot{\alpha}}
$$

where $G=\oint \frac{d z}{2 \pi i} G(z)=G_{-1 / 2}$ and similarly $\bar{G}$ are the $N=2$ world-sheet supersymmetry generators, and they have precisely the form as one would expect for a theory with $N=(2,0)$ world-sheet supersymmetry. Furthermore, from the requirement that the vertex operator produces a state in the BRST cohomology it follows that $V$ must be an $N=2$ primary of conformal weight zero and $U(1)$ charge zero, i.e. $V$ should have only single poles in the OPE with $T, G, \bar{G}$ and have a regular OPE with $J$. To analyze this condition, consider the following OPE's of $T, G, \bar{G}$ with an arbitrary function $M$ of $x_{\alpha \dot{\alpha}}$ and $\theta^{\alpha}, \bar{\theta}^{\dot{\alpha}}$

$$
\begin{aligned}
d_{\alpha}(z) M(w) & =\frac{\alpha^{\prime} \nabla_{\alpha} M(w)}{(z-w)} \\
d_{\dot{\alpha}}(z) M(w) & =\frac{\alpha^{\prime} \nabla_{\dot{\alpha}} M(w)}{(z-w)} \\
T(z) M(w) & =\frac{-\frac{1}{2} \alpha^{\prime} \nabla^{\alpha \dot{\alpha}} \nabla_{\alpha \dot{\alpha}} M(w)}{(z-w)^{2}}+\frac{\partial M(w)}{(z-w)} \\
G(z) M(w) & =\frac{1}{i \sqrt{8 \alpha^{\prime}}}\left(\frac{\alpha^{\prime} e^{i \rho} \nabla^{\gamma} \nabla_{\gamma} M(w)}{(z-w)^{2}}+\frac{2 e^{i \rho} d^{\gamma} \nabla_{\gamma} M(w)}{(z-w)}\right) \\
\bar{G}(z) M(w) & =\frac{1}{i \sqrt{8 \alpha^{\prime}}}\left(\frac{\alpha^{\prime} e^{-i \rho} \nabla^{\dot{\gamma}} \nabla_{\dot{\gamma}} M(w)}{(z-w)^{2}}+\frac{2 e^{-i \rho} d^{\dot{\gamma}} \nabla_{\dot{\gamma}} M(w)}{(z-w)}\right)
\end{aligned}
$$

where we as an intermediate step also included the OPE of the $d$ 's with $M$. From these equations we read off that the conditions for $V$ in (2.18) to be a $N=2$ primary of 
conformal weight zero are

$$
\nabla^{\alpha} \nabla_{\alpha} V_{\beta \dot{\beta}}=\nabla^{\dot{\alpha}} \nabla_{\dot{\alpha}} V_{\beta \dot{\beta}}=\nabla^{\alpha \dot{\alpha}} \nabla_{\alpha \dot{\alpha}} V_{\beta \dot{\beta}}=0
$$

In addition, $V$ should be a primary field of weight one with respect to the anti-holomorphic Virasoro algebra, yielding the additional condition

$$
\partial^{\beta \dot{\beta}} V_{\beta \dot{\beta}}=0
$$

These equations do not yet completely classify the inequivalent vertex operators of the theory, because it is possible to perform certain gauge transformations that do not change the form of $V$. The integrated vertex operator $V$ is invariant under

$$
\delta\left(V_{\alpha \dot{\alpha}} \bar{\Pi}^{\alpha \dot{\alpha}}\right)=\left[\bar{L}_{-1}, Z\right]
$$

and under

$$
\delta V_{\alpha \dot{\alpha}}=\left\{G, e^{-i \rho} X_{\alpha \dot{\alpha}}\right\}+\left\{\bar{G}, e^{i \rho} \bar{X}_{\alpha \dot{\alpha}}\right\}
$$

from which we find, up to some constants,

$$
\delta V_{\alpha \dot{\alpha}}=\nabla_{\alpha \dot{\alpha}} Z+\nabla^{\beta} \nabla_{\beta} X_{\alpha \dot{\alpha}}+\nabla^{\dot{\beta}} \nabla_{\dot{\beta}} \bar{X}_{\alpha \dot{\alpha}} .
$$

One may verify that $V_{\alpha \dot{\alpha}}$, subject to (2.20), (2.21) and with the invariance (2.24) describe the on-shell content of supergravity coupled to a tensor multiplet, with prepotential $V_{\alpha \dot{\alpha}}$ [4]. To do this verification, one chooses a Wess-Zumino gauge for $V_{\alpha \dot{\alpha}}$, after which one can count the off-shell degrees of freedom contained in $V_{\alpha \dot{\alpha}}$. There are 12 bosonic and 12 fermionic off-shell components, and by examining their spins one finds that they indeed describe conformal supergravity coupled to a tensor multiplet.

The structure of the linearized field equations and gauge fixing conditions (2.20), (2.21), and the gauge invariances in (2.24) were all dictated by the particular form of the two-dimensional theory we started with, and by the fact that the theory has an $N=2$ superconformal symmetry. Different manifestly target space supersymmetric twodimensional sigma models describing the heterotic string might yield different off-shell descriptions of supergravity, but since the sigma model we discuss here is the only known covariant description of the heterotic string, this is also the first time we do actually see string theory selecting a particular off-shell description.

Our next task is to write down the sigma model in an arbitrary curved background. To do this, we should couple the sigma model in flat space to the most general possible set of background fields. In addition, we want to compare this with the vertex operators (2.18). To see what type of coupling to background fields the latter predicts, we use the fact that for an arbitrary function $M$ of $x, \theta, \bar{\theta}$ the following identity holds

$$
\begin{aligned}
{[\bar{G},[G, M\}\}=} & 2 \Pi^{\alpha} \nabla_{\alpha} M-\frac{1}{4} d^{\alpha}\left(\nabla^{\dot{\beta}} \nabla_{\dot{\beta}} \nabla_{\alpha} M\right) \\
& +i \Pi^{\alpha \dot{\alpha}} \nabla_{\dot{\alpha}} \nabla_{\alpha} M-i d^{\dot{\alpha}}\left(\nabla^{\alpha} \nabla_{\alpha \dot{\alpha}} M\right) \\
& -\alpha^{\prime} \partial \rho \nabla^{\alpha \dot{\alpha}} \nabla_{\dot{\alpha}} \nabla_{\alpha} M
\end{aligned}
$$


which can be rewritten as

$$
\begin{aligned}
{[\bar{G},[G, M\}\}=} & \left(\Pi^{\alpha} \nabla_{\alpha} M-\Pi^{\dot{\alpha}} \nabla_{\dot{\alpha}} M\right)+\partial M-\frac{1}{2 i} \Pi^{\alpha \dot{\alpha}}\left[\nabla_{\dot{\alpha}}, \nabla_{\alpha}\right] M \\
& -\frac{1}{4}\left(d^{\alpha}\left(\bar{\nabla}^{2} \nabla_{\alpha} M\right)-d^{\dot{\alpha}}\left(\nabla^{2} \nabla_{\dot{\alpha}} M\right)\right)-\frac{1}{4} d^{\dot{\alpha}}\left(\nabla_{\dot{\alpha}} \nabla^{2}\right) M \\
& +\frac{i \alpha^{\prime}}{8}(\partial \rho)\left(\bar{\nabla}^{2} \nabla^{2}-\nabla^{2} \bar{\nabla}^{2}+8 \nabla^{\alpha \dot{\alpha}} \nabla_{\alpha \dot{\alpha}}\right) M,
\end{aligned}
$$

where $\nabla^{2}=\nabla^{\alpha} \nabla_{\alpha}$ and $\bar{\nabla}^{2}=\nabla^{\dot{\alpha}} \nabla_{\dot{\alpha}}$. Based on these observations, one is then led to propose[嘈] for the sigma model in curved space exactly the form as given in (2.12)

$$
S=\frac{1}{\alpha^{\prime}} \int d^{2} z\left(\frac{1}{2} \Pi^{\alpha \dot{\alpha}} \bar{\Pi}_{\alpha \dot{\alpha}}+d_{\alpha} \bar{\Pi}^{\alpha}+d_{\dot{\alpha}} \bar{\Pi}^{\dot{\alpha}}+\frac{1}{2} \bar{\Pi}^{A} \Pi^{B} B_{B A}-\frac{\alpha^{\prime}}{2} \bar{\partial} \rho\left(\partial \rho+a_{z}\right)\right)
$$

with the definitions in (2.10), but now with arbtitrary vielbeins and anti-symmetric tensor fields. One might imagine also introducing terms like $\partial \rho \bar{\Pi}^{A} N_{A}$ and ghost dependend terms in the sigma model, but such terms are not present in the vertex operators (the $\partial \rho$ term in (2.26) drops out when $M$ satisfies (2.20)), and are probably forbidden by world-sheet superconformal invariance (for example, $\partial \rho$ terms would break world sheet $U(1)$ invariance). Therefore we will not consider such couplings here. The generators $J, G, \bar{G}$ of the $N=2$ algebra are the same for the sigma model in curved space and in flat space (see (2.3)), but the stress energy tensor is in curved space replaced by

$$
T=\frac{1}{\alpha^{\prime}}\left(-\frac{1}{2} \Pi^{\alpha \dot{\alpha}} \Pi_{\alpha \dot{\alpha}}-d_{\alpha} \Pi^{\alpha}-d_{\dot{\alpha}} \Pi^{\dot{\alpha}}+\frac{\alpha^{\prime}}{2} \partial \rho \partial \rho\right) .
$$

As usual, the sigma model action contains potentials $B_{B A}$ and $E_{M}{ }^{A}$ rather than a prepotentials like $V_{\alpha \dot{\alpha}}$. In particular, the sigma model is manifestly space-time supersymmetric. Of course, the anti-symmetric tensor field and the vielbeins contain many more degrees of freedom than the prepotential $V_{\alpha \dot{\alpha}}$. We can at this stage already see what we expect will happen when we do a perturbative analysis of (2.12). The single poles in (2.19) arise from tree-level contractions, whereas the double poles arise from double contractions, corresponding to one-loop diagrams. Since it is the double poles that give rise to the linearized field equations for the physical components of the vertex operator, we expect that in our sigma model the equations of motion will only appear at one-loop, and that at tree level we will find a set of constraints that reduces the field content from the vielbein and anti-symmetric tensor field to the field strenghts of conformal supergravity coupled to a tensor multiplet. (As the sigma model is manifestly supersymmetric, we expect to find the field strengths rather than the prepotentials of supergravity.) Later, we will see that this is indeed what happens (in a rather subtle way), but before we do a perturbative computation in (2.12), we first have to include a Fradkin-Tseytlin term in the action to take the dilaton into account. Including the dilaton, the field content becomes that of Poincaré supergravity coupled to a tensor multiplet.

\subsection{The Fradkin-Tseytlin term}

The Fradkin-Tesytlin term in the action will be a direct generalization of the dilaton coupling $\int d^{2} z \sqrt{g} R \Phi$ of the bosonic string [10]. The dilaton is not part of the antisymmetric tensor field $B_{A B}$, because it should not couple classically in the action. As we 
will see later from the tree-level constraints, the tensor multiplet is expressed completely in terms of the torsions, and in particular the constraints break target space conformal invariance. Therefore the dilaton must be part of the remaining superfield, which is the conformal compensator. In a more conventional formulation one would use the conformal invariance to gauge fix the conformal compensator rather than the tensor multiplet, but in the sigma model it is the other way around. Something similar happens in the bosonic string: the dilaton there is the compensator for conformal transformations, a fact which is reflected in the wrong sign of the kinetic term of the dilaton. This is very particular for the 'string gauge' for target space conformal transformations that the sigma model selects. By going from the string gauge to another gauge by means of a field redefinition, it is possible to obtain a kinetic term for a scalar degree of freedom which does have the right sign. However, this scalar degree of freedom should no longer be called the dilaton, the dilaton being by definition the scalar field which counts the number of string loops. Similar statements apply to heterotic superstring and will be discussed in more detail in section 5 .

To write down the explicit form of the dilaton term, we need to know a little about $N=(2,0)$ world-sheet supergravity [11] and $N=(2,0)$ superspace. The latter contains two extra anti-commuting coordinates which we will call $\kappa$ and $\bar{\kappa}$. The two (holomorphic) superderivatives are $D=\partial_{\kappa}-\bar{\kappa} \partial$ and $\bar{D}=\partial_{\bar{\kappa}}-\kappa \partial$, whereas the supersymmetry generators are $Q=\partial_{\kappa}+\bar{\kappa} \partial$ and $\bar{Q}=\partial_{\bar{\kappa}}+\kappa \partial$. They satisfy $\{D, \bar{D}\}=-2 \partial$ and $\{Q, \bar{Q}\}=2 \partial$. If we want to identify the action of $Q$ and $\bar{Q}$ with those of $G \equiv \oint \frac{d z}{2 \pi i} G$ and $\bar{G}$, we find that a $(2,0)$ superfield is expressed in terms of its lowest components as follows

$$
\Phi=\phi+\kappa[G, \phi\}+\bar{\kappa}[\bar{G}, \phi\}+\frac{1}{2} \kappa \bar{\kappa}([G,[\bar{G}, \phi\}\}-[\bar{G},[G, \phi\}\}) .
$$

In particular, if $\phi$ is target space chiral

$$
\Phi=\phi+\kappa[G, \phi\}-\kappa \bar{\kappa} \partial \phi
$$

and, correspondingly, if $\phi$ is target space anti-chiral

$$
\bar{\Phi}=\bar{\phi}+\bar{\kappa}[\bar{G}, \bar{\phi}\}+\kappa \bar{\kappa} \partial \bar{\phi} .
$$

With these conventions, we find that the $(2,0)$ world-sheet super stress-energy tensor $\Theta$ is given by the component expansion

$$
\Theta=J-\kappa G+\bar{\kappa} \bar{G}+2 \kappa \bar{\kappa} T
$$

On a curved $(2,0)$ world sheet, the derivatives $D, \bar{D}$ and $\partial$ get replaced by covariant derivatives $\nabla_{\kappa}, \nabla_{\bar{\kappa}}$, and $\nabla_{z}$. In the case of a $(0,0)$ world-sheet, the curvature arises in the commutator $\left[\nabla_{z}, \nabla_{\bar{z}}\right] \sim R M$, where $M$ is the generator of Lorentz transformations. In the same way, the curvatures of a $(2,0)$ world-sheet can be extracted from the commutators $\left[\nabla_{\bar{\kappa}}, \nabla_{\bar{z}}\right] \sim \Sigma(M+i Y)$ and $\left[\nabla_{\kappa}, \nabla_{\bar{z}}\right] \sim \bar{\Sigma}(M-i Y)$, where $Y$ is the generator of $U(1)$ transformations, with respect to which $\kappa$ and $\bar{\kappa}$ have weights $\pm \frac{1}{2}$, and $\Sigma$ and $\bar{\Sigma}$ are respectively chiral and anti-chiral world-sheet supercurvatures. The components of $\Sigma$ look like $\Sigma \sim \chi+\kappa(R+i F)-\kappa \bar{\kappa} \partial \chi$, where $R$ and $F$ are the world-sheet curvature and $U(1)$ field strength, and $\chi$ is the gravitino field strength [11]. If now $\Phi$ and $\bar{\Phi}$ are the 
chiral and anti-chiral world-sheet superfields associated to target space chiral and antichiral superfields $\phi, \bar{\phi}$ as in (2.30) and (2.31), we can write down the Fradkin-Tseytlin term [4]

$$
\int d^{2} z d \kappa \epsilon^{-1} \Phi \Sigma+\int d^{2} z d \bar{\kappa} \bar{\epsilon}^{-1} \bar{\Phi} \bar{\Sigma}
$$

where $\epsilon$ is the $(2,0)$ world-sheet chiral density. The fields $\Phi$ and $\bar{\Phi}$ have to be chiral and anti-chiral, otherwise (2.33) would break local $(2,0)$ world-sheet supersymmetry.

The precise form of the Fradkin-Tseytlin term will not be very important to us in the remainder, since we will always work in superconformal gauge. In superconformal gauge, $\Sigma \sim i \kappa \bar{\partial} a_{z}$ and the only remnant of the Fradkin-Tseytlin term is an additional term in the action

$$
\Delta S=-\frac{i}{2} \int d^{2} z \bar{\partial}(\phi-\bar{\phi}) a_{z}
$$

In addition, the Fradkin-Tseytlin term modifies the generators of the $N=(2,0)$ superconformal algebra. This modification can easily be determined from (2.33), and reads

$$
\Theta \rightarrow \Theta+\partial(\Phi-\bar{\Phi})
$$

However, there is an additional modification of the stress-energy tensor induced by the extra term (2.34), which tells us that it is no longer $\rho$ but rather $\rho+i(\phi-\bar{\phi})$ which is the chiral boson. Together with the components of (2.35) we then find for the generators in the presence of a dilaton

$$
\begin{aligned}
J & \rightarrow J+\partial \phi-\partial \bar{\phi} \\
G \rightarrow & G-\frac{1}{\sqrt{2 \alpha^{\prime}} i} \partial\left(e^{i \rho} d^{\gamma} \nabla_{\gamma} \phi\right) \\
\bar{G} \rightarrow & \bar{G}-\frac{1}{\sqrt{2 \alpha^{\prime}} i} \partial\left(e^{-i \rho} d^{\dot{\gamma}} \nabla_{\dot{\gamma}} \bar{\phi}\right) \\
T \rightarrow & \frac{1}{\alpha^{\prime}}\left(-\frac{1}{2} \Pi^{\alpha \dot{\alpha}} \Pi_{\alpha \dot{\alpha}}-d_{\alpha} \Pi^{\alpha}-d_{\dot{\alpha}} \Pi^{\dot{\alpha}}\right. \\
& \left.+\frac{\alpha^{\prime}}{2} \partial(\rho+i(\phi-\bar{\phi})) \partial(\rho+i(\phi-\bar{\phi}))\right)-\frac{1}{2} \partial^{2}(\phi+\bar{\phi}) .
\end{aligned}
$$

Previously we required that the expansion around a flat background of the sigma model in curved background should to first order reproduce the massless vertex operators of the theory. This is true for all massless vertex operators except the dilaton (for a discussion see e.g. [12]). There is a corresponding statement for the dilaton coupling, the soft dilaton theorem [13, 14], which states that the insertion of a dilaton vertex operator in a correlation function measures the world-sheet curvature at that point, exactly as one would expect from the Fradkin-Tseytlin term (for a more recent discussion of the dilaton, see [15]). Thus one could attempt to further justify the form (2.33) by showing that in this formulation of the heterotic string there are two dilaton-type vertex operators, that respectively compute the world-sheet curvature and $U(1)$ curvature.

Instead of (2.33), there may be other possibilities to couple the dilaton. For example, in [16] the dilaton is coupled to the ghost current rather than to the world-sheet curvature. The difference between the two couplings is given by a term quadratic in the dilaton which 
through a field redefinition be absorbed in the target space metric. In either case the part linear in the dilaton is uniquely given by the Fradkin-Tseytlin term. Dilaton couplings with a different Fradkin-Tseytlin type term have been suggested in [17], but it is not clear how to implement these in the present context.

In our case we will find it convenient to redefine the $U(1)$ gauge field $a_{z}$ by

$$
a_{z} \rightarrow a_{z}+i \partial(\phi-\bar{\phi})
$$

which does not change the theory but introduces a quadratic dilaton term in the action, which now reads

$$
\begin{array}{r}
S=\frac{1}{\alpha^{\prime}} \int d^{2} z\left(\frac{1}{2} \Pi^{\alpha \dot{\alpha}} \bar{\Pi}_{\alpha \dot{\alpha}}+d_{\alpha} \bar{\Pi}^{\alpha}+d_{\dot{\alpha}} \bar{\Pi}^{\dot{\alpha}}+\frac{1}{2} \bar{\Pi}^{A} \Pi^{B} B_{B A}\right. \\
\left.-\frac{\alpha^{\prime}}{2}(\bar{\partial} \rho+i \bar{\partial}(\phi-\bar{\phi}))\left(\partial \rho+i \partial(\phi-\bar{\phi})+a_{z}\right)\right)
\end{array}
$$

After this redefinition, the action is invariant under the following local spacetime $U(1)$ transformations

$$
\begin{gathered}
\delta \phi=-\frac{1}{2} \Lambda, \quad \delta \bar{\phi}=\frac{1}{2} \Lambda, \\
\delta \Pi^{\alpha}=-\frac{1}{2} \Lambda \Pi^{\alpha}, \quad \delta \Pi^{\dot{\alpha}}=\frac{1}{2} \Lambda \Pi^{\dot{\alpha}}, \\
\delta d_{\alpha}=\frac{1}{2} \Lambda d_{\alpha}, \quad \delta d_{\dot{\alpha}}=-\frac{1}{2} \Lambda d_{\dot{\alpha}}, \quad \delta \rho=i \Lambda
\end{gathered}
$$

In addition, the action (2.38) is invariant under local target space Lorentz transformation, and the covariant derivatives $\nabla_{A}$ with a flat index $A$ contain therefore both a spin and a $U(1)$ connection (the conventions we use for these are given in appendix A); in particular the dilaton $\phi$ should be covariantly chiral, a $U(1)$ invariant statement. Both the Lorentz and $U(1)$ symmetries need to be preserved after quantization of the sigma model, in order to arrive at a non-anomalous target space theory, but both can suffer from sigma-model anomalies. One does indeed expect such anomalies to be present, due to the fields $d_{\alpha}$ and $d_{\dot{\alpha}}$, which are like chiral fermions, although their conformal weight is one. We postpone the discussion of the anomalies they cause to section 4.3, and we will for the time being assume that the Lorentz and $U(1)$ symmetries are unbroken. Notice that one necessary condition for this to hold is indeed satisfied, namely the generators of the $N=2$ algebra in (2.3) together with (2.36) are $U(1)$ and Lorentz invariant.

Having described the sigma model and some of its properties, we now turn to its perturbative analysis.

\section{The sigma model at tree level}

\subsection{Perturbation theory in the sigma model}

Before setting up the perturbation theory in $\alpha^{\prime}$ for (2.38) using a covariant background formalism, we notice that there is one immediate problem, and that is the field $\rho$. Its kinetic term does not have an explicit factor of $1 / \alpha^{\prime}$ in front, and therefore an arbitrary 
number of $\rho$-loops contributes to any given order in $\alpha^{\prime}$. In addition, in (2.38) $\rho$ couples to the other fields of the theory through $\phi-\bar{\phi}$, and it is not obvious whether or not it is possible to find explicit results for all diagrams with a fixed number of $\rho$-loops and to sum them up. Furthermore, in (2.38) the world-sheet Lagrange multiplier $a_{z}$ imposes the constraint $\bar{\partial}(\rho+i(\phi-\bar{\phi}))=0$ which is difficult to handle. Luckily, these last two problems disappear if we make the field redefinition

$$
\rho \rightarrow \rho-i(\phi-\bar{\phi})
$$

after which $\rho$ becomes a chiral boson which can be quantized exactly, and which does not interact with the other fields in the theory. Our perturbation theory will therefore consist of a hybrid combination of conventional perturbation theory for all fields except $\rho$, and exact conformal field theory results for $\rho$. After this field redefinition, the generators of the $N=2$ algebra read

$$
\begin{aligned}
J & =-i \partial \rho \\
G & =\frac{1}{i \alpha^{\prime} \sqrt{8 \alpha^{\prime}}} e^{i \rho} e^{\phi-\bar{\phi}} d^{\alpha} d_{\alpha}-\frac{1}{\sqrt{2 \alpha^{\prime}} i} \partial\left(e^{i \rho} e^{\phi-\bar{\phi}} d^{\gamma} \nabla_{\gamma} \phi\right) \\
\bar{G} & =\frac{1}{i \alpha^{\prime} \sqrt{8 \alpha^{\prime}}} e^{-i \rho} e^{\bar{\phi}-\phi} d^{\dot{\alpha}} d_{\dot{\alpha}}-\frac{1}{\sqrt{2 \alpha^{\prime}} i} \partial\left(e^{-i \rho} e^{\bar{\phi}-\phi} d^{\dot{\gamma}} \nabla_{\dot{\gamma}} \bar{\phi}\right) \\
T & \rightarrow \frac{1}{\alpha^{\prime}}\left(-\frac{1}{2} \Pi^{\alpha \dot{\alpha}} \Pi_{\alpha \dot{\alpha}}-d_{\alpha} \Pi^{\alpha}-d_{\dot{\alpha}} \Pi^{\dot{\alpha}}+\frac{\alpha^{\prime}}{2} \partial \rho \partial \rho\right)-\frac{1}{2} \partial^{2}(\phi+\bar{\phi})
\end{aligned}
$$

Next, we describe the kind of calculation we intend to do. Our goal is to obtain the equations of motion of the low-energy effective target space action that is obtained from (2.38) by integrating out all world-sheet fields. In the case of the bosonic string, the equations of motion are equivalent to finiteness of the sigma model and can therefore be obtained from the beta-functions for the background fields [18, 19, 10]. It is, due to the presence of the $\rho$-field, not obvious that a similar computation would provide the equations of motion in our case. A second complication is that finiteness of the sigma model only implies that the sigma model is conformal, and does not guarantee the full $N=(2,0)$ superconformal invariance. The latter would only follow from a standard supersymmetric $\beta$-function calculation if the model could be formulated in $N=(2,0)$ superspace on the world-sheet, which does not seem possible. In view of these complications we have chosen to employ an alternative approach, which we will compare to $\beta$-function calculations in section 4.4 .

Our approach is an extension of the one that has been developed for the bosonic string in [16]. In that paper it is shown that the equations of motion for the bosonic string can also be derived by requiring nilpotency of the BRST-operator of the sigma model through one-loop. This is in turn equivalent to demanding that the Virasoro algebra is preserved at one-loop. The equivalent statement in our case would be that the $N=2$ algebra in (2.5) is preserved at one-loop, and this is certainly something we can check. One-loop here refers to our hybrid way of doing perturbation theory, i.e. one-loop in all fields except $\rho$ and to all orders in the field $\rho$. 
To verify (2.5) at one-loop, we use a covariant background field expansion in the action of the sigma model, and so that every field gets a non-zero vacuum expectation value, except $\rho$, which we treat exactly. Next, we require that the singular parts of the one-loop expectation values of each of the pairs of operators on the left-hand sides in (2.5) matches the one-loop expectation value of the right-hand side. For example, for the Virasoro algebra, we want to verify the identity

$$
<T(z) T(w)>=\frac{c / 2}{(z-w)^{4}}+\frac{2<T(w)>}{(z-w)^{2}}+\frac{<\partial T(w)>}{(z-w)}+\text { regular }
$$

through one-loop. This computation will be desribed in section 4 , but before that we first have to describe the background field expansion of the sigma model and to analyze it at tree-level.

\subsection{Covariant background field expansion}

In the background field method all fields are decomposed in terms of background fields which satisfy the classical field equationsf and quantum fields which represent the quantum fluctuations around the background fields. We want to develop an expansion which is covariant with respect to the target space local symmetries. In our case, this means a local Lorentz and $U(1)$ covariant expansion. The traditional way to achieve such an expansion is to use Riemann normal coordinates [20]. Riemann normal coordinates are defined as follows: Let $Z_{0}$ be a point on a (super)-manifold and $y^{M}$ a tangent vector at $Z_{0}$. Let $Z(t)$ be the geodesic that satisfies $Z(0)=Z_{0}$ and $\left.\frac{d}{d t} Z(t)\right|_{t=0}=y^{M}$, then the $y^{M}$ define coordinates in a neighborhood of $Z_{0}$ by associating to $y^{M}$ the point $\left.Z\left(Z_{0} ; y^{M}\right) \equiv Z(t)\right|_{t=1}$ of $\mathcal{M}$. In Riemann normal coordinates, one labels the point $Z\left(Z_{0} ; y^{M}\right)$ by $Z_{0}^{M}+y^{M}$, and in those coordinates the geodesics are are just straight lines eminating from $Z_{0}^{M}$. In general the geodesic $Z(t)$ is given by $Z(t)=Z\left(Z_{0} ; t y^{M}\right)$.

The geodesic equation can be written as

$$
\frac{d Z^{M}}{d t} \nabla_{M} \frac{d Z^{N}}{d t}=0
$$

Now let us denote by $y^{N}\left(Z_{0} ; y^{M}\right)$ the tangent vector at $Z\left(Z_{0} ; y^{M}\right)$ obtained by parallel transporting the tangent vector $y^{N}$ at $Z_{0}$ to the point $Z\left(Z_{0} ; y^{M}\right)$ along the geodesic $Z\left(Z_{0} ; t y^{M}\right)$. In other words, $y^{N}\left(Z_{0} ; y^{M}\right)$ satisfies $y^{N}\left(Z_{0} ; 0\right)=y^{N}$ and

$$
\frac{d Z^{P}\left(Z_{0} ; t y^{M}\right)}{d t} \nabla_{P} y^{N}\left(Z_{0} ; t y^{M}\right)=0
$$

The geodesic equation implies that (3.5) is solved in particular by

$$
y^{M}\left(Z_{0} ; t y^{P}\right) \equiv \frac{d}{d t} Z^{M}\left(Z_{0} ; t y^{P}\right),
$$

which allows us to rewrite (3.5) as

$$
y^{M}\left(Z_{0} ; y^{P}\right) \nabla_{M} y^{N}\left(Z_{0} ; y^{P}\right)=0 .
$$

\footnotetext{
${ }^{5}$ If one is only interested in the 1PI effective action it is not necessary to require that the background fields satisfy the classical equations of motion; in our case we want to verify identitites like (3.3) and then it is more convenient to impose the equations of motion.
} 
In particular, in Riemann normal coordinates, the tangent space at $Z\left(Z_{0} ; y^{M}\right)=Z_{0}+y^{M}$ is naturally identified with the tangent space at $Z_{0}$, and (3.7) is solved by $y^{N}\left(Z_{0} ; y^{M}\right)=$ $y^{N}$.

The covariant background field expansion is achieved by performing a power series expansion in the Riemann normal coordinates and then covariantizing the result

$$
\tilde{\mathbf{T}}^{\prime} \equiv \mathbf{T}\left(Z_{0}+y\right)=\sum \frac{1}{n !}\left(\partial_{M_{1}} \cdots \partial_{M_{n}} \mathbf{T}\right)\left(Z_{0}\right) y^{M_{n}} \cdots y^{M_{1}}
$$

where $\mathbf{T}(Z)$ is any tensor. The coefficients of the expansion are covariantized by writing the derivatives in (3.8) as the difference of a covariant derivative and a connection piece, and by subsequently using relations between non-covariant objects, like derivatives of connections, and covariant tensors that are only valid in Riemann normal coordinates. For example, in the purely bosonic case we have in normal coordinates relations of the form

$$
\partial_{k} \Gamma_{m n}^{l}=\frac{1}{3}\left(R_{m k n}^{l}+R_{n k m}^{l}\right) .
$$

If, however, one is only interested in the expansion of scalar quantities then there is a better algorithm available 21, 22]. Observe that (3.8) can be also written as

$$
\tilde{\mathbf{T}}^{\prime}=e^{y^{M} \partial_{M}} \mathbf{T}
$$

If $\mathbf{T}=S$ is a scalar we can immediately covariantize this expression by just converting the derivative into a covariant derivative.

$$
\tilde{S}^{\prime}=e^{y^{A} \nabla_{A}} S
$$

Notice that in the exponent we also replaced curved indices $M$ by the more convenient flat indices $A$, using a vielbein to convert one index into the other: $y^{A}=y^{M} E_{M}{ }^{A}$ and $\nabla_{M}=E_{M}{ }^{A} \nabla_{A}$. Whereas $\nabla_{M}$ contained a Christoffel connection, $\nabla_{A}$ contains a spin connection, and its explicit form is given in (A.9).

If $S$ is composed of several tensors then (3.11) implies (by the chain rule) an "induced expansion" for them which is given by exactly the same formula (3.11),

$$
\mathbf{T}^{\prime}=e^{y^{A} \nabla_{A}} \mathbf{T} .
$$

Let us further define

$$
\Delta \mathbf{T}=\left[y^{A} \nabla_{A}, \mathbf{T}\right]
$$

Clearly $\mathbf{T}^{\prime}$ can be obtained by applying iteratively the operator $\Delta$.

Notice that in (3.12) we have used a different symbol than in (3.8). Both (3.12) and (3.8) are covariant, but they differ by a Lorentz rotation

$$
\tilde{\mathbf{T}}^{\prime}=\Omega\left(Z_{0} ; y\right) \mathbf{T}^{\prime}
$$

When composing tensors to construct a scalar, the dependence on the Lorentz transformation in (3.14) will drop out, and the result will be the same regardless of whether we used (3.8) or (3.12). In practise, (3.12) is much easier to use. Equation (3.12) has a simple geometrical interpretation: the left hand side is the result obtained by parallel transporting $\mathbf{T}\left(Z\left(Z_{0} ; y\right)\right)$ back from $Z\left(Z_{0} ; y\right)$ to $Z_{0}$. Again, when composing tensors to 
form a scalar, these parallel transport terms drop out, and a third, equivalent way to construct a covariant background field formalism for scalars would be to simple take $\mathbf{T}\left(Z\left(Z_{0} ; y\right)\right)$. This has the disadvantage that the expansion of a tensor $\mathbf{T}$ will contain explicit connection terms, because $\mathbf{T}\left(Z\left(Z_{0} ; y\right)\right)$ lives at $Z\left(Z_{0} ; y\right)$ whereas it is expanded in terms of objects that live at $Z_{0}$, but, again, in scalar quantities the connection terms cancel. Although we will only use (3.12), the distinction between the different approaches is important in order to understand the at first awkward looking rules like (3.21).

We are interested in carrying out the background field expansion of the action $I$ and the generators $T, G, \bar{G}, J$ of the superconformal algebra. All of them are both Lorentz and $U(1)$ scalars. Therefore, we can use (3.12). However, they are not composed directly of tensors but rather they are made up from vielbeins and connections. Hence, we need to determine the expansion of $E_{A}{ }^{M}, \omega_{A \beta}{ }^{\gamma}$ and $\Gamma_{A}$. To this end, consider $\mathbf{T}=\nabla_{A}$. Using the definitions and conventions in appendix $\mathrm{A}$ in (A.9) and (A.10), we obtain from $\Delta \nabla_{A}=\left[y^{B} \nabla_{B}, \nabla_{A}\right]$ that

$$
\begin{aligned}
\Delta E_{A}{ }^{M} & =-\left[\left(\nabla_{A} y^{B}\right)-y^{C} T_{C A}^{B}\right] E_{B}{ }^{M} \\
\Delta \omega_{A \beta}{ }^{\gamma} & =-\left[\left(\nabla_{A} y^{B}\right)-y^{C} T_{C A}^{B}\right] \omega_{B \beta}{ }^{\gamma}+y^{B} R_{B A \beta}{ }^{\gamma} \\
\Delta \Gamma_{A} & =-\left[\left(\nabla_{A} y^{B}\right)-y^{C} T_{C A}^{B}\right] \Gamma_{B}+y^{B} F_{B A}
\end{aligned}
$$

Furthermore, let $\mathbf{T}=y^{A}$, then using the geodesic equation we immediately get $\Delta y^{A}=$ 0 . Recalling that $\Pi^{A}=\partial Z^{M} E_{M}^{A}, \nabla=\Pi^{A} \nabla_{A}$, and defining

$$
\nabla y^{A}=\partial y^{A}+\Pi^{B}\left[\omega_{B \beta}{ }^{\gamma} M_{\gamma}{ }^{\beta}+\omega_{B \dot{\beta}}{ }^{\dot{\gamma}} M_{\dot{\gamma}}^{\dot{\beta}}+\Gamma_{B} Y, y^{A}\right]
$$

we finally obtain, using that $\partial Z^{M}$ evaluated at $Z^{M}\left(Z_{0} ; y\right)$ and parallel transported back to $Z_{0}$ is just $\partial Z_{0}^{M}$,

$$
\begin{aligned}
\Delta \Pi^{A} & =\nabla y^{A}-\Pi^{B} y^{C} T_{C B}{ }^{A} \\
\Delta\left(\nabla y^{A}\right) & =-y^{D} \Pi^{B} y^{C} R_{C B D}{ }^{A}-w(A) y^{A} \Pi^{B} y^{C} F_{C B}
\end{aligned}
$$

where $w(A)$ the $U(1)$ charge associated with the index $A$, i.e. $w(a)=0, w(\alpha)=1 / 2$ and $w(\dot{\alpha})=-1 / 2$.

The action (2.38) contains in addition to $\left(\Pi^{A}, \bar{\Pi}^{A}\right)$ the world-sheet fields $d_{\alpha}$ and $\rho$. Although these are world-sheet fields, one sees from the action (2.38) and the generators (3.2) that they should be viewed as being located at $Z\left(Z_{0} ; y\right)$. Correspondingly, in order to do the same covariant background field expansion for them as for the other fields in the sigma model, they should also be parallel transported to $Z_{0}$. This can be achieved using the same equation $(3.12)$ as we use for the other fields in the theory, where only the connection piece in $\nabla_{A}$ in (3.12) acts on $d_{\alpha}$ and $\rho$. On $\rho$, which is $U(1)$ neutral after the field redefinition (3.1) and also a Lorentz scalar, the connection piece acts trivially, so that $\rho$ has a trivial background field expansion, $\Delta \rho=0$. However, $d_{\alpha}$ transforms under Lorentz and $U(1)$ transformations, leading to the rule

$$
\Delta d_{\beta}=y^{A}\left(\omega_{A \beta}^{\gamma}+\frac{1}{2} \Gamma_{A} \delta_{\beta}^{\gamma}\right) d_{\gamma} .
$$

This rule is only valid to first order in $y$. To obtain the higher order terms in the expansion of $d_{\alpha}$, one should first work out $e^{y^{A} \nabla_{A}} d_{\alpha}$ to the required order and then put $\partial_{A} d_{\alpha}=0$ in 
this expansion. Although this leads to terms that explicitly depend on the connections in the background field expansion, as one already sees (3.21), the theory is still Lorentz and $U(1)$ invariant, because $d_{\alpha}$ transforms as if it were located at $Z\left(Z_{0} ; y\right)$, and the explicit expressions for the transformation rules (in terms of objects that live at $Z_{0}$ ) will contain connection pieces as well, rendering the total background field expansion Lorentz and $U(1)$ invariant.

Although (3.21) seems to be the most natural background field expansion for $d_{\alpha}$, one can always make a field redefinition of $d$ to arrive at any other background field expansion with

$$
\Delta d_{\alpha}=y^{C} U_{c \alpha}^{\beta} d_{\beta}
$$

Such field redefinitions do in principle have jacobians that can affect the one-loop properties of the sigma model. It is our point of view that one could in principle take any background field expansion of $d$ and take that as the definition of the sigma model. In a similar way one has the freedom to make a field redefinition of the quantum variable $y^{A}$. With this in mind, we will from now on also choose the trivial background field expansion for $d_{\alpha}, \Delta d_{\alpha}=0$. The advantage of this choice is that Lorentz symmetry is completely manifest, with $d_{\alpha}$ transforming naively. In addition, we will give a background expectation value $D_{\alpha}$ to $d_{\alpha}$,

$$
d_{\alpha} \rightarrow d_{\alpha}+D_{\alpha}
$$

We are now ready to perform the covariant expansion of the action $S$. For the purpose of our calculation we only need to go up to two background fields in the part quadratic in the quantum fields (denoted by $S^{(2)}$ ), and up to one background field in the part with three quantum fields (denoted by $S^{(3)}$ ). This can be seen by either a general analysis of the orders of $\alpha^{\prime}$ in the theory, of by an inspection of the Feynman diagrams that occur. Terms with 4 quantum fields and no background fields contribute only when two of the quantum fields are contracted as in diagram 34 in figure 1, but since massless tadpoles vanish in dimensional regularization, we have not explicitly given the results for these terms. The results for $S^{(2)}$ read

$$
\begin{aligned}
S^{(2)}= & \frac{1}{2} \nabla y^{a} \bar{\nabla} y^{a}+d_{\tilde{\alpha}} \bar{\nabla} y^{\tilde{\alpha}} \\
& +\frac{1}{2} \bar{\nabla} y^{a} y^{C}\left(\Pi^{B} T_{B C}{ }^{a}\right)+\frac{1}{2} \nabla y^{a} y^{C}\left(\bar{\Pi}^{B} T_{B C}{ }^{a}\right)-\frac{1}{4} \bar{\nabla} y^{C} y^{B}\left(\Pi^{a} T_{B C}{ }^{a}+2 \Pi^{A} H_{A B C}\right) \\
& +\frac{1}{2} \bar{\nabla} y^{C} y^{B}\left(T_{B C}{ }^{\tilde{\alpha}} D_{\tilde{\alpha}}\right)+d_{\tilde{\alpha}} y^{C}\left(\bar{\Pi}^{B} T_{B C}{ }^{\tilde{\alpha}}\right)-\frac{1}{4} \nabla y^{C} y^{B}\left(\bar{\Pi}^{a} T_{B C}{ }^{a}-2 \bar{\Pi}^{A} H_{A B C}\right) \\
& +\frac{1}{4} y^{B} y^{C}\left[\left(\Pi^{D} \bar{\Pi}^{a}+\bar{\Pi}^{D} \Pi^{a}\right) T_{D C B}{ }^{a}-2 \bar{\Pi}^{D} T_{D C B}{ }^{\tilde{\alpha}} D_{\tilde{\alpha}}\right. \\
& \left.\quad+\bar{\Pi}^{D}\left((-1)^{E(D+B)+C D+1} T_{C}{ }^{E a} T_{D B}{ }^{a}+H_{D C B}{ }^{E}\right) \Pi_{E}\right]
\end{aligned}
$$

whereas for $S^{(3)}$ we have

$$
\begin{aligned}
S^{(3)}= & -\frac{1}{4}\left(\bar{\nabla} y^{a} \nabla y^{B} y^{C}+\nabla y^{a} \bar{\nabla} y^{B} y^{C}\right) T_{C B}{ }^{a} \\
& +\frac{1}{3} \bar{\nabla} y^{A} \nabla y^{B} y^{C} H_{C B A}+\frac{1}{2} d_{\tilde{\alpha}} y^{B} \bar{\nabla} y^{C} T_{C B}{ }^{\tilde{\alpha}}
\end{aligned}
$$




$$
\begin{gathered}
+\frac{1}{4}\left(\bar{\nabla} y^{a} y^{B} y^{C} \Pi^{D}+\nabla y^{a} y^{B} y^{C} \bar{\Pi}^{D}\right) T_{D C B}{ }^{a}+\frac{1}{2} d_{\tilde{\alpha}} y^{B} y^{C} \bar{\Pi}^{D} T_{D C B}{ }^{\tilde{\alpha}} \\
+y^{B} y^{C} \nabla y^{D}\left[\frac{1}{12} \bar{\Pi}^{a}\left(T_{D C B}{ }^{a}+(-1)^{C D} \nabla_{C} T_{D B}{ }^{a}\right)\right. \\
\left.+\left(-\frac{1}{3} H_{D C B}{ }^{A}+\frac{1}{6} T_{D C}{ }^{E} H_{E B}{ }^{A}-\frac{1}{4} T_{D C}{ }^{e} T_{B}{ }^{A e}\right) \bar{\Pi}_{A}\right] \\
+y^{B} y^{C} \bar{\nabla} y^{D}\left[\frac{1}{12} \bar{\Pi}^{a}\left(T_{D C B}{ }^{a}+(-1)^{C D} \nabla_{C} T_{D B}{ }^{a}\right)\right. \\
\left.+\left(\frac{1}{3} H_{D C B}{ }^{A}-\frac{1}{6} T_{D C}{ }^{E} H_{E B}{ }^{A}-\frac{1}{4} T_{D C}{ }^{e} T_{B}{ }^{A e}\right) \Pi_{A}\right] \\
-\frac{1}{6} y^{B} y^{C} \bar{\nabla} y^{D}\left[T_{D C B}{ }^{\tilde{\alpha}}+(-1)^{C D} \nabla_{C} T_{D B}{ }^{\tilde{\alpha}}\right] D_{\tilde{\alpha}},
\end{gathered}
$$

where

$$
\begin{aligned}
& T_{D C B}{ }^{A}=R_{D C B}{ }^{A}+w(A) F_{D C} \delta_{B}{ }^{A}+T_{D C}{ }^{E} T_{E B}{ }^{A}+(-1)^{C D} \nabla_{C} T_{D B}{ }^{A} \\
& H_{D C B A}=\nabla_{C} H_{D B A}(-1)^{C D}-T_{C A}{ }^{E} H_{E D B}(-1)^{A(B+D)+C D}+T_{D C}{ }^{E} H_{E B A} .
\end{aligned}
$$

Next, we give the expansions of the leading parts of the generators of the superconformal algebra in (3.2). Again to the order we are interested in they are given by

$$
\begin{aligned}
T= & -\nabla y^{a} \Pi^{a}-D_{\tilde{\alpha}} \nabla y^{\tilde{\alpha}}-d_{\tilde{\alpha}} \Pi^{\tilde{\alpha}}-y^{C} \Pi^{B}\left(T_{B C}{ }^{a} \Pi_{a}-T_{B C}{ }^{\tilde{\alpha}} D_{\tilde{\alpha}}\right) \\
& -\frac{1}{2} \nabla y^{a} \nabla y^{a}-d_{\tilde{\alpha}} \nabla y^{\tilde{\alpha}} \\
& -\nabla y^{a} y^{C} \Pi^{B} T_{B C}{ }^{a}+\frac{1}{2} \nabla y^{B} y^{C} \Pi^{a} T_{B C}{ }^{a}-\frac{1}{2} \nabla y^{B} y^{C} T_{B C}{ }^{\tilde{\alpha}} D_{\tilde{\alpha}} \\
& -d_{\tilde{\alpha}} y^{B} \Pi^{C} T_{C B}{ }^{\tilde{\alpha}}-\frac{1}{2} y^{B} y^{C} \Pi^{D}\left[T_{D C B}{ }^{a} \Pi^{a}-T_{D C B}{ }^{\tilde{\alpha}} D_{\tilde{\alpha}}\right. \\
& \left.-(-1)^{E(D+B)+C D} T_{C}^{E a} T_{D B}{ }^{a} \Pi_{E}\right] \\
G= & e^{i \rho} e^{\phi-\bar{\phi}}(d+D)^{2}\left(1+y^{A} \nabla_{A}(\phi-\bar{\phi})+\frac{1}{2} y^{A} y^{B} \nabla_{B} \nabla_{A}(\phi-\bar{\phi})\right. \\
& \left.+\frac{1}{2}\left(y^{A} \nabla_{A}(\phi-\bar{\phi})\right)^{2}\right) \\
\bar{G}= & e^{-i \rho} e^{\bar{\phi}-\phi}(\bar{d}+\bar{D})^{2}\left(1+y^{A} \nabla_{A}(\bar{\phi}-\phi)+\frac{1}{2} y^{A} y^{B} \nabla_{B} \nabla_{A}(\bar{\phi}-\phi)\right. \\
& \left.+\frac{1}{2}\left(y^{A} \nabla_{A}(\bar{\phi}-\phi)\right)^{2}\right)
\end{aligned}
$$

Finally, the relevant expansions of the dilaton pieces of the superconformal algebra in (3.2) read

$$
\begin{aligned}
G_{\text {dil }}=- & \frac{1}{\sqrt{2 \alpha^{\prime} i}} \partial\left(e^{i \rho} e^{\phi-\bar{\phi}} d^{\gamma} \nabla_{\gamma} \phi+e^{i \rho} e^{\phi-\bar{\phi}} D^{\gamma} y^{A} \nabla_{A} \nabla_{\gamma} \phi\right. \\
& \left.+e^{i \rho} e^{\phi-\bar{\phi}} D^{\gamma} \nabla_{\gamma} \phi y^{A} \nabla_{A}(\phi-\bar{\phi})\right)
\end{aligned}
$$




$$
\begin{aligned}
\bar{G}_{\text {dil }}=- & \frac{1}{\sqrt{2 \alpha^{\prime} i}} \partial\left(e^{-i \rho} e^{\bar{\phi}-\phi} d^{\dot{\gamma}} \nabla_{\dot{\gamma}} \bar{\phi}+e^{-i \rho} e^{\bar{\phi}-\phi} D^{\dot{\gamma}} y^{A} \nabla_{A} \nabla_{\dot{\gamma}} \bar{\phi}\right. \\
& \left.+e^{-i \rho} e^{\bar{\phi}-\phi} D^{\dot{\gamma}} \nabla_{\dot{\gamma}} \bar{\phi} y^{A} \nabla_{A}(\bar{\phi}-\phi)\right) \\
T_{\text {dil }}= & -\frac{1}{2} \partial^{2}\left(y^{A} \nabla_{A}(\phi+\bar{\phi})\right)
\end{aligned}
$$

Besides Lorentz and $U(1)$ invariance, the background field expansion of (2.38) has an additional set of symmetries, which we denote by 'shift symmetries'. These originate in the fact that the original action (2.38) depends only on the vielbeins, not on connections. The relation between torsions, connections and vielbeins is given by (A.12). Adding a covariant tensor to any of the connections in (A.12) and subtracting a corresponding covariant term from the torsions will leave the vielbeins invariant. This 'shift symmetry' should therefore be an invariance of the action. In our case one can indeed verify that the action and the $N=2$ generators are invariant under the following non-linearly realized shift symmetry:

$$
\begin{aligned}
\delta \omega_{A B}{ }^{C} & =Y_{A B}{ }^{C} \\
\delta \Gamma_{A} & =X_{A} \\
\delta T_{A B}{ }^{C} & =Y_{[A B\}}{ }^{C}+w(C) X_{[A} \delta_{B)}{ }^{C} \\
\delta y^{A} & =-\frac{1}{2} y^{B} y^{C}\left(Y_{C B}{ }^{R}+w(A) X_{C} \delta_{B}{ }^{A}\right)+\mathcal{O}\left(y^{3}\right) \\
\delta d_{\alpha} & =\left(y^{M} Y_{M \alpha}{ }^{\beta}+\frac{1}{2} y^{A} X_{A} \delta_{\alpha}{ }^{\beta}\right)\left(d_{\beta}+D_{\beta}\right)+\mathcal{O}\left(y^{2}\right)
\end{aligned}
$$

where $X_{A}$ and $Y_{A B}{ }^{C}$ are arbitrary covariant tensors, such that $Y_{A B}{ }^{C}$ has the same symmetries as the spin connection and preserves the fact that the Lorentz group acts reducibly in target space (see (A.13)-A.16) ). In the next section we will gauge this 'shift symmetry' by putting certain torsions equal to zero. These gauge fixing conditions are usually called 'conventional constraints'.

Apart from these observations, we will completely ignore all non-covariant terms in the background field expansion, since in the absence of anomalies the final results of our computation should be Lorentz and $U(1)$ invariant (we will come back to this in section 4.3; in the bosonic case explicit calculations show all explicit dependence on the spin connection indeed drops out [23]).

\subsection{Tree-level constraints}

We are now ready to examine whether or not the sigma model has an $N=2$ algebra at tree level. By examining the orders of $\alpha^{\prime}$ in (2.5), and using exact results for $\rho$ we find that at tree level we only need to verify

$$
\begin{aligned}
T(z) T(w) & =\frac{2 T(w)}{(z-w)^{2}}+\frac{\partial T(w)}{(z-w)}+\mathcal{O}\left((z-w)^{0}\right) \\
T(z) e^{\phi-\bar{\phi}} d^{2}(w) & =\frac{2 e^{\phi-\bar{\phi}} d^{2}(w)}{(z-w)^{2}}+\frac{\partial\left(e^{\phi-\bar{\phi}} d^{2}\right)(w)}{(z-w)}+\mathcal{O}\left((z-w)^{0}\right)
\end{aligned}
$$




$$
\begin{aligned}
T(z) e^{\bar{\phi}-\phi} \bar{d}^{2}(w) & =\frac{2 e^{\bar{\phi}-\phi} \bar{d}^{2}(w)}{(z-w)^{2}}+\frac{\partial\left(e^{\bar{\phi}-\phi} \bar{d}^{2}\right)(w)}{(z-w)}+\mathcal{O}\left((z-w)^{0}\right) \\
e^{\phi-\bar{\phi}} d^{2}(z) e^{\phi-\bar{\phi}} d^{2}(w) & =\mathcal{O}\left((z-w)^{1}\right) \\
e^{\bar{\phi}-\phi} \bar{d}^{2}(z) e^{\bar{\phi}-\phi} \bar{d}^{2}(w) & =\mathcal{O}\left((z-w)^{1}\right) \\
e^{\phi-\bar{\phi}} d^{2}(z) e^{\bar{\phi}-\phi} \bar{d}^{2}(w) & =\mathcal{O}\left((z-w)^{-1}\right)
\end{aligned}
$$

where $T$ denotes here the $\rho$-indepent piece of $T$. Notice the peculiar orders of $z-w$ to which order these identities should hold; these follow from the OPE's of the exponentials of $\rho$ appearing in $G$ and $\bar{G}$.

In addition to (3.31), the generators of the $N=2$ superconformal algebra should be holomorphic, implying $\bar{\partial} T=\bar{\partial}\left(e^{\phi-\bar{\phi}} d^{2}\right)=\bar{\partial}\left(e^{\bar{\phi}-\phi} \bar{d}^{2}\right)=0$. Clearly, the presence of terms proportional to for example $(\bar{z}-\bar{w}) /(z-w)^{3}$ in the right hand side of (3.31) would imply that the fields on the left hand side are not holomorphic, but the converse need not be true, namely that the absence of nonholomorphic pieces in the OPE's implies that the currents themselves are holomorphic.

The stress-energy tensor $T$ is the Noether current associated to the symmetry

$$
\begin{aligned}
\Pi^{A} \rightarrow \Pi^{A} \partial \epsilon+\partial \Pi^{A} \epsilon, & \bar{\Pi}^{A} \rightarrow \Pi^{A} \bar{\partial} \epsilon+\epsilon \partial \bar{\Pi}^{A} \\
d^{\alpha} \rightarrow d^{\alpha} \partial \epsilon+\partial d^{\alpha} \epsilon, & d^{\dot{\alpha}} \rightarrow d^{\dot{\alpha}} \partial \epsilon+\partial d^{\dot{\alpha} \epsilon}
\end{aligned}
$$

and this immediately implies (i) that $\bar{\partial} T=0$ and (ii) that the first three OPE's in (3.31) are satisfied, as can be seen by comparing the transformations of $T, e^{\phi-\bar{\phi}} d^{2}$ and $e^{\overline{\phi-\phi}} \bar{d}^{2}$ that follow from (3.32) and (3.33) to those that are generated by $\oint \frac{d z}{2 \pi i} \epsilon(z) T(z)$ according to the OPE's (3.31). It therefore remains to analyze $e^{\phi-\bar{\phi}} d^{2}$ and $e^{\bar{\phi}-\phi} \bar{d}^{2}$.

Since it is easier to work out the conditions $\bar{\partial}\left(e^{\phi-\bar{\phi}} d^{2}\right)=\bar{\partial}\left(e^{\bar{\phi}-\phi} \bar{d}^{2}\right)=0$ than to compute the tree diagrams contributing to (3.31), we start with the former. For this we need the equations of motion of (2.38). These can be found in a very easy way by applying the operator $\Delta$ which generates the covariant background field expansion (see the previous section) once to (2.38). This yields the following field equations

$$
\begin{aligned}
0 & =\bar{\Pi}^{\tilde{\alpha}} \\
0 & =\nabla \bar{\Pi}_{a}+\bar{\nabla} \Pi_{a}-\Pi^{C} T_{C a}{ }^{b} \bar{\Pi}_{b}-\bar{\Pi}^{c} T_{c a}{ }^{b} \Pi_{b}+2 \bar{\Pi}^{c} T_{c a}{ }^{\tilde{\beta}} d_{\tilde{\beta}}+2 \Pi^{C} \bar{\Pi}^{b} H_{b C a} \\
0 & =\bar{\nabla} d_{\alpha}+\frac{1}{2}\left(\Pi^{C} T_{C \alpha}{ }^{b} \bar{\Pi}_{b}+\bar{\Pi}^{c} T_{c \alpha}{ }^{b} \Pi_{b}\right)-\bar{\Pi}^{c} T_{c \alpha}{ }^{\tilde{\beta}} d_{\tilde{\beta}}-\Pi^{C} \bar{\Pi}^{b} H_{b C \alpha}
\end{aligned}
$$

These can all be used to express $\bar{\nabla}$ (background field) in terms bilinear in the background fields, using the identity

$$
\nabla \bar{\Pi}^{A}-\bar{\nabla} \Pi^{A}=-\bar{\Pi}^{B} \Pi^{C} T_{C B}{ }^{A} .
$$

Using the equations of motion (3.36), we find that $\bar{\partial}\left(e^{\phi-\bar{\phi}} d^{2}\right)=\bar{\partial}\left(e^{\bar{\phi}-\phi} \bar{d}^{2}\right)=0$ if and only if

$$
\begin{array}{r}
T_{\tilde{\alpha} \tilde{\beta} a}-2 H_{\tilde{\alpha} \tilde{\beta} a}=0 \\
T_{a \tilde{\beta} c}+T_{c \tilde{\beta} a}=H_{a \tilde{\beta} c}=0
\end{array}
$$




$$
\begin{aligned}
& T_{a \beta}{ }^{\dot{\beta}}=T_{a \dot{\beta}}{ }^{\beta}=0 \\
& T_{a \beta}{ }^{\beta}+\nabla_{a}(\phi-\bar{\phi})=0 \\
& T_{a \dot{\beta}}^{\dot{\beta}}+\nabla_{a}(\bar{\phi}-\phi)=0
\end{aligned}
$$

Notice the explicit dilaton terms in (3.41) and (3.42). They are crucial in order that the constraints are invariant under the 'shift symmetry' mentioned in the previous section. Although it is not obvious at this stage, imposing (3.41) and (3.42) puts the theory partially on-shell. It is, however, not necessary to impose (3.41) and (3.42) in order to have a consistent tree-level theory. If (3.41) and (3.42) are not satisfied, $G$ and $\bar{G}$ satisfy equations of the form $\bar{\partial} G=U G, \bar{\partial} \bar{G}=\bar{U} \bar{G}$ for some $U$. With $U$ nonzero, it is still possible to couple the theory to world-sheet supergravity, with a modified transformation rule for the gravitino under superconformal transformations. At the linearized level, this is particularly easy to see. Consider the world-sheet action given by

$$
S=S_{\text {sigma model }}+\int d^{2} z(\nu G+\bar{\nu} \bar{G})
$$

where $\nu, \bar{\nu}$ are gauge fields for the superconformal transformations generated by $G$ and $\bar{G}$. Under such a transformation with parameters $\epsilon, \bar{\epsilon}$, the action transforms, up to irrelevant numerical factors, into

$$
\delta S=\int d^{2} z\left(\epsilon(\bar{\partial} G+U G)+\bar{\epsilon}(\bar{\partial} \bar{G}+\bar{U} \bar{G})+\delta_{\epsilon} \nu G+\delta_{\bar{\epsilon}} \bar{\nu} \bar{G}\right)
$$

Therefore, if we take $\delta_{\epsilon} \nu=\bar{\partial} \epsilon-U \epsilon$ and similarly for $\delta_{\bar{\epsilon}} \bar{\nu}$, the action is invariant.

Violating any of the other constraints (3.38)-(3.40) would imply that $\bar{\partial} G$ would no longer be proportional to $G$, and this is not acceptable. Therefore, (3.38)-(3.40) have to be imposed.

We continue by analyzing the tree-level OPE of $d^{2}$ with $\bar{d}^{2}$. There is only one diagram that can possibly contribute, which is diagram (49) in figure 1, which contains a table of all diagrams that are relevant for this paper. Diagrams with more than two external background field lines are always of order $\mathcal{O}\left((z-w)^{-1}\right)$, and are therefore not relevant for (3.31). As one sees from the the kinetic term in $S^{(2)}$ in (3.24), there is no propagator from $d_{\alpha}$ to $d_{\dot{\beta}}$, which is what would be needed to obtain a nonzero contribution from diagram (49). We conclude that diagram (49) vanishes and that the OPE of $d^{2}$ and $\bar{d}^{2}$ has the required form.

It remains to analyze the OPE of $d^{2}$ with itself, the analysis for $\bar{d}^{2}$ with itself is similar. Again, diagram (49) yields no contribution. However, we now have to include all diagrams with up to four background fields, as we need the OPE up to order $\mathcal{O}\left((z-w)^{1}\right)$. The diagrams with three or four background fields that contribute are diagrams (50), (51), (52), (55) and (59). Diagrams (53), (54) and (56) vanish as they involve the product of at least three $D$ 's.

The relevant diagrams can be worked out in a straightforward fashion in either coordinate or momentum space, and although they are completely finite there is an ambiguity in the results

\footnotetext{
${ }^{6} \mathrm{~A}$ more detailed discussion of momentum and coordinate space methods will be given in section 4 .
} 
up to an overall factor,

$$
\begin{aligned}
<e^{\phi-\bar{\phi}} d^{2}(z) e^{\phi-\bar{\phi}} d^{2}(w)>\sim & \frac{(\bar{z}-\bar{w})^{2}}{(z-w)^{2}}\left(\bar{\partial} D^{\alpha} D^{\gamma} \bar{\Theta}_{\gamma \alpha}\right) \\
& +\frac{\bar{z}-\bar{w}}{z-w}\left(\partial D^{\alpha} D^{\gamma} \bar{\Theta}_{\gamma \alpha}+\bar{\partial} D^{\alpha} D^{\gamma} \Theta_{\gamma \alpha}\right) \\
& +\left(\partial D^{\alpha} D^{\gamma} \Theta_{\gamma \alpha}\right)
\end{aligned}
$$

where we defined

$$
\begin{aligned}
\Theta_{\rho \beta} & =\frac{1}{4} T_{\rho \beta}{ }^{a} \Pi^{a}-\frac{1}{2} T_{\rho \beta}{ }^{\tilde{\alpha}} D_{\tilde{\alpha}}+\frac{1}{2} \Pi^{B} H_{B \rho \beta}-\frac{1}{2} D_{\rho} \nabla_{\beta}(\phi-\bar{\phi}) \\
\bar{\Theta}_{\rho \beta} & =\frac{1}{4} T_{\rho \beta}{ }^{a} \bar{\Pi}^{a}-\frac{1}{2} \bar{\Pi}^{B} H_{B \rho \beta} .
\end{aligned}
$$

Since the background fields satisfy the field equations, we could in principle replace $\bar{\partial} D^{\alpha}$ in (3.45) by a bilinear in the background fields, yielding terms with four background fields, that might potentially cancel against contributions from diagram (55). However, something similar is not possible for the terms containing $\partial D^{\alpha}$, and these terms should vanish by themselves, from which we deduce that $\partial D^{\alpha} D^{\gamma} \Theta_{\gamma \alpha}$ and $\partial D^{\alpha} D^{\gamma} \bar{\Theta}_{\gamma \alpha}$ should vanish completely. This leads to the constraints

$$
T_{\alpha \beta}^{c}=T_{\alpha \beta} \dot{\gamma}=H_{\alpha \beta c}=H_{\alpha \beta \dot{\gamma}}=H_{\alpha \beta \gamma}=0
$$

and

$$
T_{\alpha \beta}^{\beta}+2 \nabla_{\alpha}(\phi-\bar{\phi})=0 .
$$

The constraints (3.47) include the representation preserving constraints needed to be able to define chiral superfields in target space. On the other hand, (3.48) looks very awkward. On can easily verify that it is not invariant under the shift symmetry (3.30). Apparently, we have broken the shift symmetry in this tree level calculation. One may wonder how this is possible, since one could also have done a Poisson bracket calculation in the sigma model without using any background field expansion, in which case the shift symmetry is manifest. However, a Poisson bracket calculation for the $\rho$-independent part of the sigma model action would provide no information about the regular part of the OPE of $e^{\phi-\bar{\phi}} d^{2}$ with itself, which is precisely where the problematic term (3.48) came from.

One explanation of the ambiguities that underly (3.48) is as follows. Imagine adding a term to the action of the form

$$
\int d^{2} z\left(\bar{\nabla} D_{\gamma}+\ldots\right)\left(y^{\alpha} y^{\beta} S_{\beta \alpha}^{\gamma}\right)
$$

where $\left(\bar{\nabla} D_{\gamma}+\ldots\right)$ is the field equation (3.36) with $d$ replaced by $D$. Since $D$ satisfies the field equation, the extra term in (3.49) is zero. On the other hand, if one partially integrates the $\bar{\nabla}$ in $\bar{\nabla} D_{\gamma}$, one gets a series of vertices with two background fields, and precisely one vertex with one background fields, namely

$$
\int d^{2} z\left(-2 D_{\gamma} \bar{\nabla} y^{\alpha} y^{\beta} S_{\beta \alpha}^{\gamma}\right)
$$


If we include this vertex in the calculation, we find an extra contribution in (3.48) proportional to $S_{\alpha \beta}{ }^{\beta}$. This represents an inherent ambiguity in our calculation, and since we unfortunately do not know of any manifest shift symmetric scheme to compute things, we will simply choose $S_{\alpha \beta}{ }^{\gamma}$ so as to cancel (3.48) completely. Of course, to be self-consistent, we have to use the same vertex (3.50) also in our one-loop calculation.

Another way to look at this ambiguity is to observe that on the one hand $\bar{\nabla} D$ consists of terms bilinear in the background fields, but if one in a diagram encounters a propagator $(\bar{\partial})^{-1}$ and integrates this against $\bar{\nabla} D$, one can obtain contributions containing a single $D$. Clearly, a better understanding of these issues would be desirable.

We are left with diagrams (55) and (59) to analyze. Before doing so, we will first try to simplify these remaining calculations as much as possible, by supplementing the constraints obtained so far ((3.38), (3.39), (3.40) and (3.47)) with a maximal set of conventional constraints, and by subsequently solving the Bianchi identities for the connections $\nabla_{A},\left\{\nabla_{A},\left\{\nabla_{B}, \nabla_{C}\right\}\right\}+$ cycl. $=0$. This will further constrain the torsions and curvatures and provide us with the largest possible set of constraints implied by (3.38), (3.39), (3.40) and (3.47).

Quite remarkably, it turns out that (3.38), (3.39), (3.40) and (3.47) plus a maximal set of conventional constraints is already sufficient to completely solve the Bianchi identities and reduce the field content of the theory to that of conformal supergravity coupled to a linear multiplet. This in complete accordance with the analysis of the vertex operators in section (2.3), see in particular the discussion at the end of section (2.3), where we argued that all contraints should appear at tree level and the field equations should appear at one-loop.

Let us now determine a maximal set of conventional constraints. All but one of the conventional constraints can be viewed as a gauge fixing of the shift symmetry (3.30) discussed previously.

Recall that the parameters of the shift symmetry are covariant tensors $X_{A}$ and $Y_{A B}{ }^{C}$, where $Y_{A B}{ }^{C}$ has the same symmetries as the spin connection and preserves the fact that the Lorentz group acts reducibly in target space (see (A.13)-(A.16)). We can not use this to put the spin connection and $U(1)$ connection equal to zero, since zero is not a globally well-defined connection, but we can use it to put some torsions equal to zero. To determine how many torsion coefficients can be removed we analyze the spin content of the covariant tensors $X$ and $Y$. The tensor $Y_{\alpha \beta}{ }^{\gamma}$ contains a spin-3/2 and a spin-1 piece and can be used to remove the torsion $T_{\alpha \beta}{ }^{\gamma}$ completely. The tensor $Y_{\dot{\alpha} \beta}{ }^{\gamma}$ removes the symmetric part $T_{\dot{\alpha}\left(\beta^{\gamma}\right)}$ of the torsion whereas $X_{\dot{\alpha}}$ removes the remaining antisymmetric part $T_{\dot{\alpha} \beta}{ }^{\beta}$. Similarly, $Y_{a \beta} \gamma$ is used to put $\left.T_{a(\beta} \gamma\right)$ equal to zero, and we use $X_{a}$ to put $T_{a \beta}{ }^{\beta}-T_{a \dot{\beta}} \dot{\beta}$ equal to zero. To summarize, we impose the following conventional contraints:

$$
T_{\alpha \beta}^{\gamma}=T_{\dot{\alpha} \beta}^{\gamma}=T_{\alpha \dot{\beta}}^{\dot{\gamma}}=T_{\dot{\alpha} \dot{\beta}}^{\dot{\gamma}}=T_{a(\beta}{ }^{\gamma)}=T_{a(\dot{\beta}}{ }^{\dot{\gamma})}=T_{a \beta}{ }^{\beta}-T_{a \dot{\beta}} \dot{\beta}=0 .
$$

There is still one more conventional constraint, of a somewhat different origin, that we can impose. One can always redefine the vielbeins by performing a local Lorentz tranformation, since it is not the vielbein itself but only the metric $G_{M N}=E_{M}{ }^{A} E_{N A}$ that appears in the action. The Lorentz group acts reducible in target space, in other 
words independently on the indices $\alpha, \dot{\alpha}$ and $a$. Using a local Lorentz rotation that acts only on the vector indices $a$, we can always achieve that

$$
T_{\alpha \dot{\beta}}^{c}=c \delta_{\alpha}^{\gamma} \delta_{\dot{\beta}}^{\dot{\gamma}}
$$

where $c$ is a constant. After choosing this gauge for the vector piece of the Lorentz gauge transformations, Lorentz transformations acting on the spinor indices must be accompagnied by one on the vector indices in order to preserve (3.52). This leads to the identifications (A.13) and (A.15). Comparing with the exact result for flat superspace in (2.15) we find that $c=-2 i$, and this will be confirmed by our one-loop calculations in the remaining sections. Equation (3.38) now implies

$$
H_{\alpha \dot{\alpha} b}=-i C_{\alpha \beta} C_{\dot{\alpha} \dot{\beta}}
$$

in accordance with (2.17).

We are now ready to solve the Bianchi identities that will reduce the field content to that of conformal supergravity coupled to a tensor multiplet. The involved algebra is rather tedious and here we just present the final result, postponing the details to appendix B

$$
\begin{aligned}
\left\{\nabla_{\alpha}, \nabla_{\beta}\right\}= & 0, \\
\left\{\nabla_{\alpha}, \nabla_{\dot{\beta}}\right\}= & -2 i \nabla_{\alpha \dot{\beta}}-4 i H_{\dot{\beta} \gamma} M_{\alpha}^{\gamma}+4 i H_{\dot{\gamma} \alpha} M_{\dot{\beta}}^{\dot{\gamma}}+4 i H_{\dot{\beta} \alpha} Y, \\
{\left[\nabla_{\alpha}, \nabla_{b}\right]=} & -2 \nabla_{\beta} H_{\dot{\beta} \gamma} M_{\alpha}^{\gamma} \\
& +\left[-2 i C_{\alpha \beta} \bar{W}_{\dot{\beta} \dot{\gamma}}^{\dot{\delta}}+C_{\dot{\beta} \dot{\gamma}}\left(\nabla_{(\alpha} H^{\dot{\delta}}{ }_{\beta)}-\frac{1}{3} C_{\alpha \beta} \nabla^{\epsilon} H^{\dot{\delta}}{ }_{\epsilon}\right)\right] M_{\dot{\delta}}^{\dot{\gamma}} \\
& +2 \nabla_{\beta} H_{\dot{\beta} \alpha} Y \\
{\left[\nabla_{a}, \nabla_{b}\right]=} & -2 H_{\dot{\alpha} \beta} \nabla_{\alpha \dot{\beta}} \\
& +\left[\frac{i}{2} C_{\alpha \beta} \nabla_{(\dot{\alpha}} H_{\dot{\beta})}^{\gamma}+C_{\dot{\alpha} \dot{\beta}}\left(-\frac{i}{6} C^{\gamma}{ }_{(\alpha \mid} \nabla^{\dot{\epsilon}} H_{\dot{\epsilon} \mid \beta)}+W_{\alpha \beta}{ }^{\gamma}\right)\right] \nabla_{\gamma} \\
& +\left[C _ { \dot { \alpha } \dot { \beta } } \left(\frac{1}{24} \nabla_{(\alpha} W_{\beta \gamma}{ }^{\delta)}+\frac{1}{4}\left(C^{\delta}{ }_{\alpha} \nabla_{(\beta \mid \dot{\epsilon}} H^{\dot{\epsilon}}{ }_{\mid \gamma)}+\alpha \leftrightarrow \beta\right)\right.\right. \\
& \left.\left.+\frac{i}{6} C_{\alpha \gamma} C_{\beta}^{\delta} \nabla^{\dot{\epsilon}} \nabla^{\epsilon} H_{\dot{\epsilon} \epsilon}\right)+\frac{i}{2} C_{\alpha \beta} \nabla_{\gamma} \nabla_{(\dot{\alpha}} H_{\dot{\beta})} \delta\right] M_{\delta}^{\gamma} \\
& \left.-\frac{i}{2} C_{\alpha \beta} \nabla^{\delta} \nabla_{(\dot{\alpha}} H_{\dot{\beta}) \delta} Y+\text { c.c. }\right\}
\end{aligned}
$$

where 'c.c.' denotes our definition of complex conjugation, see appendix $\mathrm{A}, W_{\alpha \beta \gamma}$ is completely symmetric chiral superfield,

$$
\nabla_{\dot{\delta}} W_{\alpha \beta \gamma}=0
$$

and $H_{\dot{\alpha} \beta}$ is defined as follows (see also appendix B)

$$
H_{a b c}=C_{\gamma \alpha} C_{\dot{\gamma} \dot{\beta}} H_{\dot{\alpha} \beta}-C_{\gamma \beta} C_{\dot{\gamma} \dot{\alpha}} H_{\dot{\beta} \alpha}
$$


$W_{\alpha \beta \gamma}$ and $H_{\dot{\alpha} \beta}$ satisfy the following differential relations

$$
\nabla_{a} H^{a}=0, \nabla^{\gamma} W_{\gamma \alpha \beta}=\frac{i}{6} \nabla_{(\alpha \mid} \nabla^{\dot{\gamma}} H_{\dot{\gamma} \mid \beta)}+\frac{i}{2} \nabla^{\dot{\gamma}} \nabla_{(\alpha \mid} H_{\dot{\gamma} \mid \beta)}, \quad \nabla^{\beta} \nabla_{\beta} H_{a}=0 .
$$

Furthermore, all the components of the field strength $H_{A B C}$ vanish except the ones that are given in (3.53) and (3.59). Compactly, we have

$$
T_{A B c}+(-1)^{A B} 2 H_{A B c}=0 .
$$

A similar supergravity algebra has been obtained in [24].

From (3.54)-(3.57) we can read off how all torsions and curvatures can be expressed in terms of $H_{\dot{\alpha} \beta}$ and $W_{\alpha \beta \gamma}$. In particular, the background field expansion for the action will then contain only these two fields, and this is the form of the action with the fewest number of vertices and therefore the most suitable one to use in calculations.

One can verify that with maximally simplified form of the action the remaining tree diagrams (55) and (59) yield no contribution, which concludes the tree-level analysis of the theory. We next turn our attention to one-loop calculations.

\section{The sigma model at one-loop}

\subsection{Coordinate space and momentum space techniques}

The starting point for our calculations will be the covariant background field expansion for the action, with all the constraints and explicit expressions obtained from the treelevel constraints and the Bianchi identities inserted in it. In addition, as we explained before, we will for the time being drop all explicit connection terms. The part of the action quadratic in the quantum fields given in (3.24) contains a piece

$$
S_{\text {kin }}=\frac{1}{\alpha^{\prime}} \int d^{2} z\left(\frac{1}{2} \partial y^{a} \bar{\partial} y^{a}+d_{\tilde{\alpha}} \bar{\partial} y^{\tilde{\alpha}}\right)
$$

which we take as the kinetic term, since $S_{\text {kin }}$ describes free fields for which we know the propagators explicitly. The remainder of (3.24) will together with (3.25) provide the vertices of the theory.

The propagators obtained from (4.1) are in coordinate space given by

$$
\begin{gathered}
<y^{a}(z) y^{b}(w)>=\alpha^{\prime} \delta^{a b} G(z, w) \equiv-\alpha^{\prime} \delta^{a b} \log |z-w|^{2} \\
<d_{\alpha}(z) y^{\beta}(w)>=\frac{\alpha^{\prime} \delta_{\alpha}{ }^{\beta}}{(z-w)}=-\alpha^{\prime} \delta_{\alpha}{ }^{\beta} \partial_{z} G(z, w)
\end{gathered}
$$

It is now straightforward to write down coordinate space expressions for the Feynman diagrams given in figure 1 . We are only going to compute the contributions to $<T(z) T(w)>,<T(z) G(w)>$ etc. which involve at most two background fields (or more precisely, where the sum of the conformal weights of the background fields is at most two). For $\langle G(z) \bar{G}(w)>$ this is all we need (cf. (3.31)), as it is for $\langle T(z) T(w)>$ by virtue of its symmetry under $z \leftrightarrow w$. Thus, the only contributions we are missing 
are those to $<T(z) G(w)>$ and $<T(z) \bar{G}(w)>$ with three background fields and those to $<G(z) G(w)>$ and $<\bar{G}(z) \bar{G}(w)>$ with four background fields. It is a very tedious calculation to determine these contributions, and in addition we believe that they will not lead to any new equations, although we have no general proof of this.

In diagrams with two background fields, we can assume that the background fields are independent of the coordinates, or in momentum space, that they have zero momentum. If we would make a Taylor expansion of the background fields around a fixed point on the world-sheet, all derivatives of the background fields would have higher conformal weight and can be ignored. For diagrams with one or zero background fields one has to be more careful and expand everything up to the relevant order. In addition, there may be ambiguities in this similar to those discussed below (3.48), but these particular ambiguities don't play a role in the part of the calculation we present below.

In coordinate space, we then encounter various integrals not containing background fields, like for example

$$
\int d^{2} u \frac{1}{z-u} \frac{1}{u-w}
$$

Such integrals can then be manipulated using the fact that they are translationally invariant, using partial integrations inside the integral and using identities such as

$$
\partial_{\bar{z}} \frac{1}{z-w}=\delta^{(2)}(z-w) .
$$

Here, $\delta^{(2)}$ is the delta function with respect to our measure $d^{2} z$ which was defined as $d x d y / \pi$ with $z=x+i y$. For our example (4.4) one then finds

$$
\int d^{2} u \frac{1}{z-u} \frac{1}{u-w}=\frac{\bar{z}-\bar{w}}{z-w}
$$

by writing $1 /(u-w)$ as $\bar{\partial}_{\bar{u}}((\bar{u}-\bar{w}) /(u-w))$, using a partial integration and (4.5). One has to be very careful using such naive manipulations, since one-loop diagrams can have divergences, and this can sometimes lead to ambiguous answers. For example, diagram (9) can lead to integrals like

$$
\int d^{2} u d^{2} v \frac{1}{z-u} \frac{1}{u-v} \delta^{(2)}(v-w) \delta^{(2)}(u-w)
$$

If we first do the $u$ integral, we get

$$
\int d^{2} v \frac{1}{z-w} \frac{1}{w-v} \delta^{(2)}(v-w)=-\frac{1}{2} \frac{1}{z-w} \int d^{2} v \bar{\partial}_{\bar{v}} \frac{1}{(v-w)^{2}}=0 .
$$

whereas first integrating over $v$ leads to

$$
\int d^{2} u \frac{1}{z-u} \frac{1}{2} \bar{\partial}_{\bar{u}} \frac{1}{(u-w)^{2}}=\int d^{2} u \delta^{(2)}(u-z) \frac{1}{2} \frac{1}{(u-w)^{2}}=\frac{1}{2} \frac{1}{(z-w)^{2}} .
$$

This ambiguity reflects the fact that we should regularize the (relatively convergent) integrals. Although there are proposals for a consistent regularization in coordinate space [25], these are not particularly useful in our case. In our case, we have found indications that it might be possible to formulate a set of rules in coordinate space that allow one to 
express every integral in terms of unambiguous integrals and only one particular integral whose evaluation in coordinate space seems to be very difficult,

$$
D_{2}=\int d^{2} u \delta^{2} v \frac{1}{z-u}\left[\delta^{(2)}(u-v)\right]^{2} \frac{1}{v-w} .
$$

The precise value of this integral then depends on the choice of regularization prescription (in dimensional regularization it is $\left.\frac{1}{6(z-w)^{2}}\right)$, but its precise value is not important because one can always choose finite local counterterms in the generators of the $N=2$ algebra so that in the final result $D_{2}$ does not appear. Unfortunately, we have not been able to make these statements more precise, and therefore we have chosen to use dimensional regularization in momentum space instead.

In dimensional regularization, we immediately run into two problems. First, there are terms in the action coming from the antisymmetric tensor field in (2.38), and these contain two-dimensional anti-symmetric tensors $\epsilon_{i j}$ when written in a world-sheet covariant way. Second, there are 'chiral' world-sheet fields like $d_{\alpha}$ and $y^{\alpha}$. We have chosen the following version of dimensional regularization to deal with these problems. First of all, we keep all interactions in exactly two dimensions, and in particular the $\epsilon$-tensor will remain in two dimensions. Secondly, we view $d_{\alpha}$ as a vector field on the world-sheet with only a $z$-component, and we will in other dimensions still view it as a vector field with only a component in the $z$-direction. Besides this, the kinetic terms will be taken in $2-2 \epsilon$ dimensions. With such a type of regularization, one has to be careful when going beyond one loop, in which case evanescent counterterms have to be taken into account (see [26] for a detailed discussion of the two-loop renormalization of a two-dimensional sigma model, including problems with the $\epsilon$ tensor in two dimensions)]. We will not further discuss these issues here, since we will only be doing a one-loop calculation.

In the same way as we defined the measure $d^{2} z$ as $d x d y / \pi$, we will define $d^{2} k$ also as $d k_{x} d k_{y} / \pi$, and it turns out that with this choice we never see any explicit factors of $\pi$ in our calculation. Then $G(z, w)$ has the following representation

$$
G(z, w)=\int d^{2} k \frac{e^{i k(z-w)+i \bar{k}(\bar{z}-\bar{w})}}{|k|^{2}}
$$

and the propagators in momentum space read

$$
\begin{aligned}
& <y^{a}(k) y^{b}(l)>=\alpha^{\prime} \delta^{a b} \delta^{(2)}(k+l) \frac{1}{|k|^{2}} \\
& <d_{\alpha}(k) y^{\beta}(l)>=\alpha^{\prime} \delta_{\alpha}^{\beta} \delta^{(2)}(k+l) \frac{-i k}{|k|^{2}}
\end{aligned}
$$

where $k$ is shorthand notation for $k_{z}$, the $z$-component of $k$, and in dimensional regularization we keep $k$ in one dimension, and only continue the denominators in (4.12) outside two dimensions. To work out the Feynman diagrams we write down the corresponding expression in momentum space, continue to $d=2-2 \epsilon$ dimensions, and include a factor $\Gamma(1-\epsilon)(4 \pi)^{-\epsilon}(2 \pi)^{2 \epsilon}$ for each loop, which removes a lot of unwanted constants like

\footnotetext{
${ }^{7}$ There exist several other approaches to regularize the integrals that appear in chiral and non-local two-dimensional field theories, such as analytic regularization [27, exponential regularization [28] and Pauli-Villars [29]. The situation beyond one-loop is unclear, but at one-loop we expect each of these to yield equivalent answers.
} 
the Euler constant, see [26]. The $d$-dimensional measure $d^{d} k$ is equal to the standard $d$-dimensional measure divided by $\pi$. The relevant integrals come out as follows

$$
\begin{aligned}
& \Gamma(1-\epsilon)(4 \pi)^{-\epsilon}(2 \pi)^{2 \epsilon} \int d^{d} p \frac{p^{a} \bar{p}^{b}}{\left[|p|^{2}\right]^{\alpha}\left[|p-k|^{2}\right]^{\beta}}= \\
& =k^{a+1-\alpha-\beta} \bar{k}^{b+1-\alpha-\beta}\left|k^{2} / \mu^{2}\right|^{-\epsilon} \frac{\Gamma[1-\epsilon] \Gamma[1-\epsilon-\alpha+b]}{\Gamma[\alpha] \Gamma[\beta] \Gamma[2-\alpha-\beta+b-\epsilon]} \\
& \times \sum_{r=0}^{a}\left(\begin{array}{c}
a \\
r
\end{array}\right) \frac{\Gamma[2-\alpha-\beta+b+r-\epsilon] \Gamma[\alpha+\beta-1-r+\epsilon] \Gamma[1-\epsilon-\beta+r]}{\Gamma[2-2 \epsilon-\alpha-\beta+r+b]}
\end{aligned}
$$

as one proves by differentiating both sides with respect to $k$ and $\bar{k}$, starting from the known result for $a=b=0$. The parameter $\mu$ is the usual dimensional regularization mass parameter.

We have worked out all our diagrams in momentum space using (4.13), and then afterwards reexpressed them in coordinate space using the following translation table

$$
\begin{aligned}
-\frac{k^{3}}{3 \bar{k}^{3}} & \leftrightarrow \frac{(\bar{z}-\bar{w})^{2}}{(z-w)^{4}} \\
\frac{k^{2}}{2 \bar{k}^{2}} & \leftrightarrow \frac{\bar{z}-\bar{w}}{(z-w)^{3}} \\
-\frac{k}{\bar{k}} & \leftrightarrow \frac{1}{(z-w)^{2}} \\
\frac{k}{\epsilon \bar{k}}+\frac{k}{\bar{k}}\left(1-\log \left(|k|^{2} / \mu^{2}\right)\right) & \leftrightarrow \frac{G(z, w)}{(z-w)^{2}}
\end{aligned}
$$

The first three follow by Fourier transformation, the last one, which contains an $1 / \epsilon$ divergence, was chosen by comparing the result for $\langle y(z) y(w)><\partial y(z) \partial y(w)>$ in coordinate space and momentum space. In the right hand side one could in principle have chosen a different finite term proportional to $k / \bar{k}$, but since all divergences will cancel in our calculation, this would not make any difference for the final answers.

There is one more important thing we have to discuss before we present the results of our calculation. Namely, we have dropped in the expansion of $G$ and $\bar{G}$ in (3.28) all terms that came from the background field expansion of $e^{\phi-\bar{\phi}}$ and $e^{\bar{\phi}-\phi}$. Including these terms leads to a number of additional contributions in all OPE's except that of $T$ with $T$, each of which contain the dilaton. However, we claim that one can systematically remove all these terms. For this one needs (i) one-loop counterterms, (ii) ambiguities similar to the one discussed below (3.48) and (iii) the possibility to choose different background field expansions for $d$. The precise details are rather cumbersome and will be reported elsewhere [30]. We suspect there may be a better explanation in which the shift symmetry in (3.30) plays a crucial role, but for the time being all this remains rather mysterious.

Having described how we performed them, we now turn to the results of our calculations. 


\subsection{One-loop results}

Below we tabulate the contributions of diagrams (1)-(19) to $<G(z) \bar{G}(w)>$, $<$ $G(z) G(w)>,<T(z) G(w)>$ and $\langle T(z) T(w)>$. We postpone the discussion of the other diagrams for the time being; most of them do not give any contribution, except for the ones involving an insertion of the dilaton terms in (3.29), and these will be tabulated separately.

Denoting by $I_{d}$ the contribution of diagram number $d$, the non-zero diagrams for $<G(z) \bar{G}(w)>$ come out as follows (note that we have also taken the $\rho$-contributions into account, whose only effect is to shift the order of the poles by one, and $\lambda$ is the coefficient in front of $\left.G, \lambda=1 /\left(i \alpha^{\prime} \sqrt{8 \alpha^{\prime}}\right)\right)$

$$
\begin{aligned}
I_{4} & =\frac{1}{(z-w)}\left(8 \lambda^{2} \alpha^{\prime 2} \Pi^{a} \Pi^{a}\right) \\
I_{10} & =\frac{1}{(z-w)}\left(+16 \lambda^{2} \alpha^{\prime 2} D_{\beta} \Pi^{\beta}-\lambda^{2} \alpha^{\prime 2} D^{\alpha} R_{\alpha \dot{\gamma}}^{\dot{\gamma}} D_{\dot{\beta}}-\frac{1}{2} \lambda^{2} \alpha^{\prime 2} D^{\alpha} D^{\dot{\gamma}} F_{\dot{\gamma} \alpha}\right) \\
I_{15} & =\frac{1}{(z-w)}\left(16 \lambda^{2} \alpha^{\prime 2} D_{\dot{\beta}} \Pi^{\dot{\beta}}-\lambda^{2} \alpha^{\prime 2} D^{\dot{\alpha}} R^{\gamma}{ }_{\dot{\alpha} \gamma}{ }^{\beta} D_{\beta}-\frac{1}{2} \lambda^{2} \alpha^{\prime 2} D^{\alpha} D^{\dot{\gamma}} F_{\dot{\gamma} \alpha}\right)
\end{aligned}
$$

We have also computed all one-loop contributions to $<G(z) \bar{G}(w)>$, without imposing any constraints, except (3.38)-(3.42), and found many more terms contributing to < $G(z) \bar{G}(w)>$, but the result remained finite, i.e. the terms proportional to $G(z, w)$ canceled each other. This is a reflection of the fact that when $G$ and $\bar{G}$ are conserved quantities, their OPE should be finite. Adding up the contributions we get

$$
<G(z) \bar{G}(w)>=\frac{1}{(z-w)}\left(2 T(w)+\frac{2 i}{\alpha^{\prime}} D^{\alpha} D^{\dot{\beta}} H_{\dot{\beta} \alpha}\right)
$$

The first term is precisely the term we expect (see (2.5)). The second term will be removed by choosing a different background field expansion for $d$. We defer a furhter discussion of the background field expansion for $d$ and the one-loop counterterms till after we have discussed all OPE's.

None of the diagrams (1)-(19) contributes to $<G(z) G(w)>$ or $<\bar{G}(z) \bar{G}(w)>$, in agreement with (2.5).

Next we turn to $\langle T(z) G(w)>$. Below are the results for this computation, and we have extracted a factor of $\lambda \alpha^{\prime} e^{i \rho} e^{\phi-\bar{\phi}}$ To obtain the contributions to $\langle T(z) G(w)>$, one should still multiply everything by this factor.

$$
\begin{aligned}
I_{5}= & \frac{(\bar{z}-\bar{w})}{(z-w)^{3}}\left(-2 \bar{\Pi}^{d} R_{d}{ }^{\beta}{ }^{\alpha} D_{\alpha}-\bar{\Pi}^{d} F_{d}^{\gamma} D_{\gamma}\right) \\
I_{7}= & \frac{1}{(z-w)^{2}}\left(\Pi^{d} R_{d}{ }^{\beta}{ }^{\alpha} D_{\alpha}+\Pi^{\dot{\delta}} R_{\dot{\delta}}{ }^{\beta}{ }^{\alpha} D_{\alpha}+\frac{1}{2} \Pi^{d} F_{d}^{\gamma} D_{\gamma}\right. \\
& \left.\quad+\frac{1}{2} \Pi^{\dot{\delta}} F_{\dot{\delta}}^{\gamma} D_{\gamma}-8 \Pi_{\dot{\alpha}} \Pi^{\dot{\alpha}}\right) \\
I_{10}= & \frac{(\bar{z}-\bar{w})}{(z-w)^{3}}\left(\left(+2 \bar{\Pi}^{d} R_{d}{ }^{\beta}{ }^{\alpha} D_{\alpha}+\bar{\Pi}^{d} F_{d}^{\gamma} D_{\gamma}\right)\right.
\end{aligned}
$$




$$
\begin{aligned}
& +\frac{1}{(z-w)^{2}}\left(-\frac{1}{2} \Pi^{d} R_{d}^{\beta} \beta^{\alpha} D_{\alpha}-\frac{1}{2} \Pi^{\dot{\delta}} R_{\dot{\delta}}^{\beta}{ }^{\alpha}{ }^{\alpha} D_{\alpha}-\frac{1}{4} \Pi^{d} F_{d}^{\gamma} D_{\gamma}\right. \\
& \left.-\frac{1}{4} \Pi^{\dot{\delta}} F_{\dot{\delta}}^{\gamma} D_{\gamma}+8 \Pi_{\dot{\alpha}} \Pi^{\dot{\alpha}}\right) \\
& I_{14}=\frac{(\bar{z}-\bar{w})}{(z-w)^{3}}\left(-2 i \bar{\Pi}^{c} T_{c,}{ }^{\gamma}, \dot{\beta}, D_{\gamma}\right) \\
& I_{15}=\frac{(\bar{z}-\bar{w})}{(z-w)^{3}}\left(\bar{\Pi}^{a}\left(\frac{1}{2} R_{b a}^{\gamma b}+3 i T_{a,}{ }^{\gamma}{ }_{\dot{\beta}}{ }^{\dot{\beta}}+R_{a}{ }^{\beta \gamma}{ }_{\beta}-\frac{1}{2} F_{a}{ }^{\gamma}\right) D_{\gamma}\right) \\
& +\frac{1}{(z-w)^{2}}\left(\Pi^{a}\left(-\frac{1}{4} R^{\gamma b}{ }_{b a}+\frac{i}{2} T_{a,{ }^{\gamma}{ }^{\prime},}{ }^{\dot{\beta}}\right) D_{\gamma}\right) \\
& I_{18}=\frac{1}{(z-w)^{2}}\left(3 i \Pi^{c} T_{c,}{ }^{\gamma}{ }_{\dot{\beta}}{ }^{\dot{\beta}} D_{\gamma}\right) \\
& I_{19}=\frac{1}{(z-w)^{2}}\left(-\frac{13 i}{6} \Pi^{c} T_{c,}{ }^{\gamma} \dot{\beta},{ }^{\dot{\beta}} D_{\gamma}\right)
\end{aligned}
$$

Adding up all diagrams we obtain

$$
\begin{aligned}
<T(z) G(w)>= & \frac{\bar{z}-\bar{w}}{(z-w)^{3}} \bar{\Pi}^{a}\left(R_{a}{ }^{\beta}{ }^{\gamma}-\frac{1}{2} F_{a}^{\gamma}+\frac{1}{2} R_{b a}^{\gamma b}+i T_{a,{ }^{\gamma}{ }^{\gamma},}{ }^{\dot{\beta}}\right) D_{\gamma} \\
+ & \frac{1}{(z-w)^{2}}\left[\Pi ^ { a } \left(\frac{1}{2} R_{a}{ }^{\beta}{ }^{\gamma}-\frac{1}{4} R_{b a}^{\gamma b}+\frac{1}{4} F_{a}{ }^{\gamma}\right.\right. \\
& \left.\left.\quad+\frac{4}{3} i T_{a,}{ }_{\dot{\beta},}{ }^{\dot{\beta}}\right) D_{\gamma}+\Pi^{\dot{\alpha}}\left(\frac{1}{2} R_{\dot{\alpha}}{ }^{\beta}{ }^{\gamma}+\frac{1}{4} F_{\dot{\alpha}}{ }^{\gamma}\right) D_{\gamma}\right]
\end{aligned}
$$

Inserting the expicit expressions of the curvatures and torsions from (B.24) we get

$$
\begin{aligned}
<T(z) G(w)> & =\frac{\bar{z}-\bar{w}}{(z-w)^{3}}\left[-4 \bar{\Pi}^{a} \nabla_{\alpha} H_{\dot{\alpha}}^{\gamma} D_{\gamma}\right] \\
& +\frac{1}{(z-w)^{2}}\left[-\frac{17}{6} \Pi^{a} \nabla_{\alpha} H_{\dot{\alpha}}^{\gamma} D_{\gamma}+4 i \Pi^{\dot{\alpha}} H_{\dot{\alpha}}^{\gamma} D_{\gamma}\right]
\end{aligned}
$$

Finally, we list the results for $\langle T(z) T(w)>$. Due to the symmetry of this expectation value under $z \leftrightarrow w$, a diagram and its mirror image (like diagrams (2) and (7)) give identical contributions, and this is reflected in the following table

$$
\begin{aligned}
I_{1}= & \frac{G(z, w)}{(z-w)^{2}}\left(-\left(\Pi^{c} H_{c m n}-\frac{1}{2} T_{m n}{ }^{\tilde{\alpha}} D_{\tilde{\alpha}}\right)^{2}\right) \\
& +\frac{1}{(z-w)^{2}}\left(\left(\Pi^{c} H_{c m n}-\frac{1}{2} T_{m n}{ }^{\tilde{\alpha}} D_{\tilde{\alpha}}\right)^{2}\right. \\
I_{2}= & \frac{G(z, w)}{(z-w)^{2}}\left(2\left(\Pi^{c} H_{c m n}-\frac{1}{2} T_{m n}{ }^{\tilde{\alpha}} D_{\tilde{\alpha}}\right)^{2}\right) \\
& +\frac{1}{(z-w)^{2}}\left(-4\left(\Pi^{c} H_{c m n}-\frac{1}{2} T_{m n}{ }^{\tilde{\alpha}} D_{\tilde{\alpha}, \beta \dot{\alpha}}{ }^{\beta}\right)^{2}\right)
\end{aligned}
$$




$$
\begin{aligned}
& I_{3}=I_{2} \\
& I_{4}=\frac{1}{(z-w)^{2}}\left(2\left(\Pi^{c} H_{c m n}-\frac{1}{2} T_{m n}{ }^{\tilde{\alpha}} D_{\tilde{\alpha}}\right)^{2}\right) \\
& I_{5}=\frac{(\bar{z}-\bar{w})}{(z-w)^{3}}\left(-\Pi^{d} \bar{\Pi}^{a}\left(\frac{1}{2} R_{d}{ }^{m}{ }_{m a}+\frac{1}{2} R_{a}{ }^{m}{ }_{m d}+\nabla^{m} H_{a m d}\right)-\frac{1}{2} \Pi^{\tilde{\delta}} \bar{\Pi}^{a} R_{\tilde{\delta}}{ }^{m}{ }^{a}\right. \\
& +i \bar{\Pi}^{d} T_{d,}{ }^{\beta \dot{\beta}},\left(\beta \Pi_{\dot{\beta})}+2 \bar{\Pi}^{d}\left(\frac{1}{2} \nabla^{m} T_{d m}{ }^{\tilde{\alpha}}-H_{d m e} T_{e m}{ }^{\tilde{\alpha}}\right) D_{\tilde{\alpha}}\right) \\
& I_{6}=\frac{G(z, w)}{(z-w)^{2}}\left(-4\left(\Pi^{c} H_{c m n}-\frac{1}{2} T_{m n}{ }^{\tilde{\alpha}} D_{\tilde{\alpha}}\right)^{2}\right) \\
& +\frac{1}{(z-w)^{2}}\left(6\left(\Pi^{c} H_{c m n}-\frac{1}{2} T_{m n}{ }^{\tilde{\alpha}} D_{\tilde{\alpha}}\right)^{2}\right) \\
& I_{7}=\frac{1}{(z-w)^{2}}\left(\Pi^{d} \Pi^{a}\left(\frac{1}{2} R_{d c c a}-4 H_{d e c} H_{c e a}\right)\right. \\
& \left.+\frac{1}{2} \Pi^{\tilde{\delta}} \Pi^{a} R_{\tilde{\delta} c c a}+\Pi^{d}\left(H_{d e c} T_{c e}^{\tilde{\alpha}}+\frac{1}{2} \nabla_{c} T_{c d}{ }^{\tilde{\alpha}}\right) D_{\tilde{\alpha}}\right) \\
& I_{8}=I_{7} \\
& I_{9}=\frac{(\bar{z}-\bar{w})}{(z-w)^{3}}\left(-i \bar{\Pi}^{c}\left(\Pi_{\nu} T_{c}^{n}{ }_{\nu}+\Pi_{\dot{\nu}} T_{c}^{n}{ }_{\nu}\right)\right) \\
& +\frac{G(z, w)}{(z-w)^{2}}\left(+2\left(\Pi^{c} H_{c m n}-\frac{1}{2} T_{m n}{ }^{\tilde{\alpha}} D_{\tilde{\alpha}}\right)^{2}\right) \\
& +\frac{1}{(z-w)^{2}}\left(-\left(-\Pi^{c} H_{c m n}+\frac{1}{2} T_{m n}{ }^{\tilde{\alpha}} D_{\tilde{\alpha}}\right)\left(-3 \Pi^{c} H_{c m n}+\frac{1}{2} T_{m n}{ }^{\tilde{\alpha}} D_{\tilde{\alpha}}\right)\right) \\
& I_{10}=\frac{(\bar{z}-\bar{w})}{(z-w)^{3}}\left(\Pi_{m} \bar{\Pi}_{a}\left(\frac{1}{4} R_{m n n a}+\frac{3}{4} R_{a n n m}+\frac{1}{2} \nabla_{n} H_{n m a}-\frac{1}{2} \nabla^{\tilde{\gamma}} T_{a m \tilde{\gamma}}\right)\right. \\
& +\Pi^{\beta} \bar{\Pi}^{a}\left(-\frac{1}{4} R_{m \beta m a}+\frac{3 i}{2} T_{a m}{ }^{\dot{\mu}} \delta_{\beta}{ }^{\mu}+\frac{1}{2} R_{a}{ }^{\gamma} \beta \gamma-\frac{1}{4} F_{a \beta}\right) \\
& +\Pi^{\dot{\beta}} \bar{\Pi}^{a}\left(-\frac{1}{4} R_{m \dot{\beta} m a}+\frac{3 i}{2} T_{a m}{ }^{\mu} \delta_{\dot{\beta}}^{\dot{\mu}}+\frac{1}{2} R_{a}{ }_{\dot{\beta} \dot{\gamma}}+\frac{1}{4} F_{a \dot{\beta}}\right) \\
& \left.+D^{\tilde{\gamma}} \bar{\Pi}^{a}\left(\nabla_{m} T_{m a \tilde{\gamma}}+2 H_{a e m} T_{m e \tilde{\gamma}}\right)\right) \\
& +\frac{1}{(z-w)^{2}}\left(\Pi_{m} \Pi_{a}\left(\frac{1}{8} R_{n m n a}+\frac{3}{8} R_{a n m n}+H_{\text {nem }} H_{\text {nae }}\right)\right. \\
& +\Pi_{m} \Pi^{\alpha}\left(\frac{3}{8} R_{\text {onmn }}+\frac{1}{8} R_{\text {nonm }}\right) \\
& +\Pi_{m} \Pi^{\dot{\alpha}}\left(\frac{3}{8} R_{\dot{\alpha} n m n}+\frac{1}{8} R_{\text {ninmm }}\right) \\
& \left.+\Pi_{m} D^{\tilde{\alpha}} \frac{1}{2}\left(H_{n e m} T_{n e \tilde{\alpha}}+\nabla_{n} T_{m n \tilde{\alpha}}\right)\right) \\
& I_{13}=\frac{G(z, w)}{(z-w)^{2}}\left(-\left(\Pi^{c} H_{c m n}-\frac{1}{2} T_{m n}{ }^{\tilde{\alpha}} D_{\tilde{\alpha}}\right)^{2}\right)
\end{aligned}
$$




$$
\begin{aligned}
& +\frac{1}{(z-w)^{2}}\left(\left(\Pi^{c} H_{c m n}-\frac{1}{2} T_{m n}{ }^{\tilde{\alpha}} D_{\tilde{\alpha}}\right)^{2}+3 i \Pi_{(\nu \mid} \bar{\Pi}^{c} T_{c}{ }^{n} \mid \dot{\nu}\right) \\
I_{14} & =I_{9} \\
I_{15} & =I_{10} \\
I_{18} & =I_{13} \\
I_{19} & =\frac{G(z, w)}{(z-w)^{2}}\left(-\left(\Pi^{c} H_{c m n}-\frac{1}{2} T_{m n}{ }^{\tilde{\alpha}} D_{\tilde{\alpha}}\right)^{2}\right) \\
& +\frac{1}{(z-w)^{2}}\left(\left(\Pi^{c} H_{c m n}-\frac{1}{2} T_{m n}{ }^{\tilde{\alpha}} D_{\tilde{\alpha}}\right)^{2}\right. \\
& \left.\quad-\frac{13 i}{6}\left(\Pi_{\dot{\nu}} T_{m}{ }^{n}{ }_{\nu}+\Pi_{\nu} T_{m}{ }^{n} \dot{\nu}\right) \Pi_{m}\right)
\end{aligned}
$$

Adding up all contributions we get

$$
\begin{gathered}
<T(z) T(w)>=\frac{\bar{z}-\bar{w}}{(z-w)^{3}}\left[\Pi^{a} \bar{\Pi}^{b}\left(R_{b c c a}+\nabla_{\tilde{\gamma}} T_{b a}{ }^{\tilde{\gamma}}\right)\right. \\
+\Pi^{\beta} \bar{\Pi}^{a}\left(R_{a}^{\gamma}{ }_{\beta \gamma}-\frac{1}{2} F_{a \beta}\right) \\
+\Pi^{\dot{\beta}} \bar{\Pi}^{a}\left(R_{a}^{\dot{\gamma}} \dot{\beta} \dot{\gamma}+\frac{1}{2} F_{a \dot{\beta}}\right) \\
\left.+D^{\tilde{\gamma}} \bar{\Pi}^{b}\left(\nabla_{a} T_{a b \tilde{\gamma}}-2 H_{b d e} T_{d e \tilde{\gamma}}\right)\right] \\
+\frac{1}{(z-w)^{2}}\left[-\frac{i}{6}\left(\Pi^{\dot{\alpha}} \Pi^{c} T_{c, \beta \dot{\alpha}}{ }^{\beta}+\Pi^{\alpha} \Pi^{c} T_{c, \alpha \dot{\beta}} \dot{\beta}\right)\right. \\
\left.+4 \Pi^{a} \Pi^{b} H_{a c d} H_{b c d}\right]
\end{gathered}
$$

After some simplifications we obtain

$$
\begin{aligned}
&<T(z) T(w)>= \frac{\bar{z}-\bar{w}}{(z-w)^{3}}\left[\Pi^{a} \bar{\Pi}^{b} R_{b c c a}\right. \\
&+\Pi^{\beta} \bar{\Pi}^{a}\left(-2 \nabla_{\alpha} H_{\dot{\alpha} \beta}\right)+\Pi^{\dot{\beta}} \bar{\Pi}^{a}\left(2 \nabla_{\dot{\alpha}} H_{\dot{\beta} \alpha}\right) \\
&\left.+D^{\tilde{\gamma}} \bar{\Pi}^{b}\left(\nabla_{a} T_{a b \tilde{\gamma}}-2 H_{b d e} T_{d e \tilde{\gamma}}\right)\right] \\
&+\frac{1}{(z-w)^{2}}\left[\frac{1}{6} \Pi^{\alpha} \Pi^{b} \nabla_{\beta} H_{\dot{\beta} \alpha}-\frac{1}{6} \Pi^{\dot{\alpha}} \Pi^{b} \nabla_{\dot{\beta}} H_{\dot{\alpha} \beta}\right. \\
&\left.+4 \Pi^{a} \Pi^{b} H_{a c d} H_{b c d}\right]
\end{aligned}
$$

We now move to the dilaton sector. Since the dilaton corrections (3.29) to the superconformal generators enter with an extra $\alpha^{\prime}$ one only has to compute tree graphs. To $<G(z) G(w)>$ only diagrams (60) and (61) contribute, and we find

$$
I_{60}=I_{61}=\frac{1}{(z-w)^{3}}\left(4 \lambda^{2} \alpha^{2} e^{2 i \rho} e^{\phi-\bar{\phi}} D^{2} \nabla^{2} e^{\phi-\bar{\phi}}\right) .
$$

To cancel this term, one can add a one-loop counterterm to the action of the form $\Delta S \sim$ $\int d^{2} z \nabla^{2} e^{\phi-\bar{\phi}} \Pi^{\alpha} \bar{\Pi}_{\alpha}$, which does not interfere with any of the other one-loop calculations. 
If one, however, takes the point of view that it should not be allowed to add one-loop counterterms containing the dilaton to the theory, then one is forced to require $\nabla^{2} e^{\phi-\bar{\phi}}=$ 0 . Interestingly enough, we will see later that the low-energy effective action does have $\nabla^{2} e^{\phi-\bar{\phi}}=0$ as one of its equations of motion, so that either point of view is consistent with our further results.

It is rather easy to check that there is no dilaton contribution to $\langle G(z) \bar{G}(w)\rangle$. In the tree graph the pole due to the contraction between $d_{\alpha}$ and $y^{\alpha}$ is cancelled by the $\rho$ contribution and the result is regular.

The dilaton contributions to $<T(z) G(w)>$ are as follows (again a factor of $\lambda \alpha^{\prime}$ has been extracted).

$$
\begin{aligned}
I_{61}= & \frac{1}{(z-w)^{2}}\left(-2 e^{i \rho} D^{\alpha} \partial\left(\nabla_{\alpha} e^{\phi-\bar{\phi}}\right)\right) \\
I_{62}= & \frac{1}{(z-w)^{3}} 2 e^{i \rho} e^{\phi-\bar{\phi}} D^{\alpha} \nabla_{\alpha} \phi \\
+ & \frac{\bar{z}-\bar{w}}{(z-w)^{3}} 2 e^{i \rho} e^{\phi-\bar{\phi}} D^{\alpha} \bar{\partial}\left(\nabla_{\alpha} \phi\right) \\
I_{63}= & \frac{1}{(z-w)^{3}}\left(-4 e^{i \rho} D^{\alpha} \nabla_{\alpha} e^{\phi-\bar{\phi}}\right) \\
& +\frac{\bar{z}-\bar{w}}{(z-w)^{3}}\left(-4 e^{i \rho} \bar{\partial}\left(D^{\alpha}\right) \nabla_{\alpha} e^{\phi-\bar{\phi}}\right) \\
& +\frac{1}{(z-w)^{2}}(-2)\left(\partial\left(e^{i \rho}\right) D^{\alpha} \nabla_{\alpha} e^{\phi-\bar{\phi}}+e^{i \rho} D^{\alpha} \partial\left(\nabla_{\alpha} e^{\phi-\bar{\phi}}\right)+2 e^{i \rho}\left(\partial D^{\alpha}\right) \nabla_{\alpha} e^{\phi-\bar{\phi}}\right) \\
I_{65}= & -I_{67}=4 i \Pi^{\dot{\alpha}} \Pi_{\alpha \dot{\alpha}} \partial_{w}\left(\frac{1}{z-w} e^{i \rho} \nabla^{\alpha} e^{\phi-\bar{\phi}}\right) \\
I_{69}= & \frac{1}{(z-w)^{3}}\left(2 e^{i \rho} D^{\alpha} \nabla_{\alpha} e^{\phi-\bar{\phi}}\right) \\
& +\frac{1}{(z-w)^{2}}\left(\partial\left(e^{i \rho} D^{\alpha} \nabla_{\alpha} e^{\phi-\bar{\phi}}\right)-2 \partial\left(e^{i \rho}\right) D^{\alpha} \nabla_{\alpha} e^{\phi-\bar{\phi}}\right)
\end{aligned}
$$

Here, diagram (69) refers to a contraction of the term $\frac{1}{2} \partial \rho \partial \rho$ in $T$ with the dilaton term in $G$. Adding up these contributions we get that the cubic poles cancel, $G_{\text {dil }}$ has conformal weight $3 / 2$ and the remaining non-holomorphic part is equal to

$$
\frac{\bar{z}-\bar{w}}{(z-w)^{3}} 2 e^{i \rho} e^{\phi-\bar{\phi}} D^{\alpha} \bar{\Pi}^{b} \nabla_{b} \nabla_{\alpha} \phi
$$

We are now ready to write down the first field equation. As in the bosonic string the field equations are determined from the non-holomorphic part. The holomorphic part determines the counterterms to the currents. From (4.18) and (4.24) we obtain the following field equation

$$
2 \nabla_{\alpha} H_{\dot{\alpha} \beta}=\nabla_{\beta} \nabla_{a}(\phi+\bar{\phi}) .
$$

The dilaton contribution to $\langle T(z) T(w)>$ is tabulated below

$$
I_{62}+I_{63}=\frac{1}{(z-w)^{2}} 2\left(-\frac{1}{2} \partial^{2}(\phi+\bar{\phi})\right)
$$




$$
\begin{array}{r}
+\frac{\bar{z}-\bar{w}}{(z-w)^{3}}\left(2 \Pi^{A} \bar{\Pi}^{b} \nabla_{b} \nabla_{A}(\phi+\bar{\phi})-\bar{\partial} \partial(\phi+\bar{\phi})\right) \\
I_{64}=I_{65}=\frac{1}{(z-w)^{2}}\left(-\frac{1}{2} \Pi^{b} T_{b c}{ }^{\tilde{\alpha}} D_{\tilde{\alpha}} \nabla_{c}(\phi+\bar{\phi})\right) \\
I_{66}=I_{67}=\frac{1}{(z-w)^{2}}\left(\frac{1}{2} \Pi^{b} T_{b c}{ }^{\tilde{\alpha}} D_{\tilde{\alpha}} \nabla_{c}(\phi+\bar{\phi})\right) \\
+\frac{\bar{z}-\bar{w}}{(z-w)^{3}}\left(-D_{\tilde{\alpha}} \bar{\Pi}^{b} T_{c b}{ }^{\tilde{\alpha}} \nabla_{c}(\phi+\bar{\phi})\right. \\
\left.+\Pi^{a} \bar{\Pi}^{b}\left(T_{a b}{ }^{\gamma} \nabla_{\gamma} \phi+T_{a b}{ }^{\dot{\gamma}} \nabla_{\dot{\gamma}} \bar{\phi}\right)\right)
\end{array}
$$

Adding up these contributions we obtain that $T_{\text {dil }}$ has conformal weight 2 plus the following non-holomorphic contribution

$$
\frac{\bar{z}-\bar{w}}{(z-w)^{3}}\left(\Pi^{A} \bar{\Pi}^{b} \nabla_{b} \nabla_{A}(\phi+\bar{\phi})+\Pi^{a} \bar{\Pi}^{b} T_{a b} \tilde{\gamma} \nabla_{\tilde{\gamma}}(\phi+\bar{\phi})+D^{\tilde{\alpha}} \bar{\Pi}^{b} T_{c b \tilde{\alpha}} \nabla_{c}(\phi+\bar{\phi})\right)
$$

Thus, from (4.21) and (4.27) follows that the following three equations should hold

$$
\begin{aligned}
& 2 \nabla_{\alpha} H_{\dot{\alpha} \beta}=\nabla_{\beta} \nabla_{a}(\phi+\bar{\phi}), \\
& R_{a b b c}=-\nabla_{a} \nabla_{c}(\phi+\bar{\phi})+T_{a c} \tilde{\delta} \nabla_{\tilde{\delta}}(\phi+\bar{\phi}), \\
& \nabla_{a} T_{a b} \tilde{\gamma}-2 H_{b d e} T_{d e} \tilde{\gamma}=T_{b a} \tilde{\gamma} \nabla_{a}(\phi+\bar{\phi}) .
\end{aligned}
$$

Observe that (4.28) is precisely equation (4.25)! Even more, the last two equations follow from the first as we now show!

Let us first introduce some notation. Let $T_{A}=\nabla_{A}(\phi+\bar{\phi})$. We also define

$$
\begin{aligned}
& \beta_{\beta a}=2 \nabla_{\alpha} H_{\dot{\alpha} \beta} ; \quad \beta_{a c}=R_{a b b c} \\
& \beta_{b \tilde{\gamma}}^{D}=\nabla_{a} T_{a b \tilde{\gamma}}-2 H_{b d e} T_{d e \tilde{\gamma}}
\end{aligned}
$$

In this notation equations (4.28) read

$$
\begin{aligned}
& \beta_{\beta a}=\nabla_{a} T_{\beta} \\
& \beta_{a c}=-\nabla_{a} T_{c}+T_{a c}^{\tilde{\delta}} T_{\tilde{\delta}} \\
& \beta_{b \tilde{\gamma}}^{D}=T_{b a \tilde{\gamma}} T_{a}
\end{aligned}
$$

We shall show that (4.33) follows from (4.32) by differentiating once and (4.34) by differentiating twice. In the process we will need a few identities that we tabulate below.

$$
\begin{aligned}
& {\left[\nabla_{\dot{\alpha}}, \nabla_{b}\right] V_{\alpha}=-2 i T_{a b}{ }^{\gamma} V_{\gamma},} \\
& {\left[\nabla_{\alpha}, \nabla_{b}\right] V_{\gamma}=2 C_{\gamma \alpha} \nabla_{\beta} H_{\dot{\beta}}{ }^{\delta} V_{\delta},} \\
& \left\{\nabla^{\alpha}, \nabla^{\dot{\alpha}}\right\} T_{\gamma}=-2 i\left(\nabla^{\alpha \dot{\alpha}} T_{\gamma}-2 H^{\dot{\alpha} \beta} T_{\beta} C^{\alpha}{ }_{\gamma}\right) \\
& T_{\alpha \dot{\alpha}, \beta \dot{\beta}, \gamma}-T_{\gamma \dot{\alpha}, \beta \dot{\beta}, \alpha}=i C_{\alpha \gamma} \nabla_{\dot{\beta}} H_{\dot{\alpha} \beta},
\end{aligned}
$$




$$
\begin{gathered}
\nabla^{\alpha} T_{\alpha \dot{\alpha}, \beta \dot{\beta}, \gamma}=2 \nabla_{b} H_{\dot{\alpha} \gamma}-i \nabla_{\dot{\alpha}} \nabla_{\beta} H_{\dot{\beta} \gamma} \\
R_{a b b c}=\frac{1}{2}\left[i C_{\alpha \gamma} C_{\dot{\alpha} \dot{\gamma}} \nabla^{\dot{\epsilon}} \nabla^{\epsilon} H_{\dot{\epsilon} \epsilon}+C_{\dot{\alpha} \dot{\gamma}} \nabla_{(\alpha \mid \dot{\epsilon}} H^{\dot{\epsilon}}{ }_{\mid \gamma)}-C_{\alpha \gamma} \nabla_{\epsilon(\dot{\alpha}} H_{\dot{\gamma})}{ }^{\epsilon}\right. \\
\left.\quad+\frac{i}{2}\left(\left[\nabla_{\alpha}, \nabla_{\dot{\alpha}}\right] H_{\dot{\gamma} \gamma}+\alpha \leftrightarrow \gamma+\dot{\alpha} \leftrightarrow \dot{\gamma}\right)\right], \\
\nabla_{a} T_{a c \dot{\delta}}=\frac{1}{2} \nabla^{\alpha}\left[\left\{\nabla_{\gamma}, \nabla_{\dot{\gamma}\}}\right\} H_{\dot{\delta} \alpha}-\nabla_{\alpha} \nabla_{\dot{\gamma}} H_{\dot{\delta} \gamma}\right] \\
\quad+4 H^{\dot{\alpha} \alpha} T_{\gamma \dot{\alpha}, \alpha \dot{\alpha}, \dot{\delta}}-2 i H_{\dot{\delta}}^{\alpha} \nabla_{\gamma} H_{\dot{\gamma} \alpha} \\
\nabla^{\alpha} \nabla_{\dot{\beta}} \nabla^{\gamma} H_{\dot{\delta} \alpha}=\nabla^{\alpha} \nabla_{\dot{\delta}} \nabla^{\gamma} H_{\dot{\beta} \alpha} .
\end{gathered}
$$

In these identities $V_{\tilde{\alpha}}$ is assumed to have the $U(1)$ charge of its index, namely $1 / 2$ if the index is undotted and $-1 / 2$ if the index is dotted. One may use the first three identities to derive how (anti-)commutators act on tensors. However, one has to be careful when the $U(1)$ charge of the tensor is different from the one its indices indicate. Such an example is the torsion $T_{a b \gamma}$ that has $U(1)$ charge $-1 / 2$ and not $1 / 2$. In such cases it is preferable to work out the commutators directly. However, when the tensor involved has the same $U(1)$ charge as its indices the identities may be used safely.

We are now ready to derive equations (4.33) and (4.34) from (4.32). Equation (4.33) is derived by differentiating (4.32) by $\nabla_{\alpha}$ and then adding to the resulting equation the complex conjugate one. Then the result follows from (4.40). To get equation (4.34) we start by defining the following object

$$
\begin{aligned}
\Omega_{\alpha \dot{\beta} \dot{\beta}}= & \frac{i}{2}\left[\nabla_{\beta} \nabla_{\dot{\beta}} T_{\alpha}+\nabla_{\dot{\beta}} \nabla_{\beta} T_{\alpha}-\nabla_{\alpha} \nabla_{\beta} T_{\dot{\beta}}-\nabla_{\alpha} \nabla_{\dot{\beta}} T_{\beta}\right] \\
& +2 C_{\alpha \beta} H_{\dot{\beta}}{ }^{\delta} T_{\delta}
\end{aligned}
$$

Using the definition of $T_{\alpha}$ one may prove that $\Omega_{\alpha \beta \dot{\beta}}$ is equal to zero. Nevertheless, we momentarily ignore this fact and treat $T_{a}$ as a tensor that only satisfies (4.32). We compute the quantity $C^{\alpha \beta} \nabla_{c} \Omega_{\alpha \beta \dot{\beta}}$. The idea is to commute $\nabla_{c}$ all the way to the right and then to use (4.32). After some rather tedious algebra that involves use of the identities (4.35)-(4.42) we get

$$
\begin{aligned}
C^{\alpha \beta} \nabla_{c} \Omega_{\alpha \dot{\beta} \dot{\beta}}= & \frac{i}{2} C^{\alpha \beta}\left(\nabla_{\beta} \nabla_{\dot{\beta}} \beta_{\alpha c}+\nabla_{\dot{\beta}} \nabla_{\beta} \beta_{\alpha c}-\nabla_{\alpha} \nabla_{\beta} \beta_{\dot{\beta} c}-\nabla_{\alpha} \nabla_{\dot{\beta}} \beta_{\beta c}\right) \\
& 4 H_{\dot{\beta}}{ }^{\delta} \beta_{\delta c}-2 T_{c, \dot{\beta}}^{\alpha \dot{\alpha}} \nabla_{(\dot{\alpha}} T_{\alpha)}+T_{\alpha \dot{\beta}, c}{ }^{\delta} \nabla^{(\alpha} T_{\delta)} .
\end{aligned}
$$

Using now the definition of $T_{A}$ we see that the last term vanishes, whereas the one but the last is equal to $4 i T_{a c \dot{\beta}} \nabla_{a}(\phi+\bar{\phi})$ which is just (up to the factor of $4 i$ ) the right hand side of (4.34). To complete the proof we have to show that

$$
\beta_{b \tilde{\gamma}}^{D}=-\frac{1}{8} C^{\alpha \beta}\left(\nabla_{\beta} \nabla_{\dot{\beta}} \beta_{\alpha c}+\nabla_{\dot{\beta}} \nabla_{\beta} \beta_{\alpha c}-\nabla_{\alpha} \nabla_{\beta} \beta_{\dot{\beta} c}-\nabla_{\alpha} \nabla_{\dot{\beta}} \beta_{\beta c}\right)+i H_{\dot{\beta}}^{\delta} \beta_{\delta c}
$$

This follows upon using (4.41) and (4.42).

Finally, let us rewrite the equation (4.28) in a form that it will appear in the next section. Observe that the left hand side of (4.28) can be rewitten as $\left[\nabla_{a}, \nabla_{\beta}\right](\phi-\bar{\phi})$. It follows that the field equation is equal to

$$
\nabla_{\gamma} \nabla_{a}(\phi-\bar{\phi})=0
$$


Having discussed the field equations we now turn to the remaining holomorphic pieces in the OPE's. We already discussed the $\langle G(z) G(w)>$ previously. In the $\langle T(z) T(w)>$ in (4.21) there are several holomorphic terms, each of which can be easily cancelled by adding the following one-loop counterterms to $T$

$$
T \rightarrow T-\frac{1}{12} \Pi^{\alpha} \Pi^{b} \nabla_{\beta} H_{\dot{\beta} \alpha}+\frac{1}{12} \Pi^{\dot{\alpha}} \Pi^{b} \nabla_{\dot{\beta}} H_{\dot{\alpha} \beta}-2 \Pi^{a} \Pi^{b} H_{a c d} H_{b c d} .
$$

This leaves an unwanted holomorphic term in $\langle G(z) \bar{G}(w)>$ of the form (see 4.15)

$$
\frac{1}{(z-w)}\left(\frac{2 i}{\alpha^{\prime}} D^{\alpha} D^{\dot{\beta}} H_{\dot{\beta} \alpha}\right)
$$

and in $<T(z) G(w)>$ that looks like

$$
\frac{1}{(z-w)^{2}}\left[-3 \Pi^{a} \nabla_{\alpha} H_{\dot{\alpha}}^{\gamma} D_{\gamma}+4 i \Pi^{\dot{\alpha}} H_{\dot{\alpha}}^{\gamma} D_{\gamma}\right]
$$

It seems impossible to cancel (4.49) by means of a counterterm. The remaining possibility is then to try to modify the background field expansion of $d$ in such a way as to cancel (4.49). Suppose we modify the background field expansion of $d$ as follows

$$
\left(d_{\alpha}+D_{\alpha}\right) \rightarrow\left(d_{\alpha}+D_{\alpha}\right)\left(1+y^{A} y^{B} N_{B A}\right)
$$

A straightforward calculation reveals that by taking $N_{a \gamma}=N_{\gamma a}=\frac{3}{2} \nabla_{\alpha} H_{\dot{\alpha} \gamma}$ and $N_{\alpha \dot{\alpha}}=$ $-N_{\dot{\alpha} \alpha}=2 i H_{\dot{\alpha} a}$ one can indeed remove (4.49) completely. A pleasant side effect of this new background field expansion for $d$ is that it also at the same time completely removes (4.48), while it does not affect any of the other results. While everything at one-loop now seems to work, it would certainly be nice to have a further, maybe even geometrical, understanding of the right background field expansion of $d$.

\subsection{Lorentz and $U(1)$ anomalies}

In this section, we discuss the anomalies of the world-sheet sigma model. The two symmetries of the theory, local Lorentz and local $U(1)$ invariance, can be discussed at the same time. We certainly expect the local Lorentz and $U(1)$ gauge transformations to be anomalous, due to the presence of the 'chiral' fields $d_{\tilde{\alpha}}, y^{\tilde{\alpha}}$ which, from the world-sheet point of view, closely resemble chiral fermions. Chiral fermions are the usual source of sigma model anomalies [31]. In the RNS formalism of the heterotic string, the local Lorentz and gauge anomalies of the low-energy effective action are closely related to the world-sheet sigma model anomalies and the latter can be cancelled by a Green-Schwarz mechanism [32, 33, 34. Since our sigma model is supposedly a reformulation of the RNS string, a similar anomaly cancellation should be possible in our case as well, as we will now demonstrate.

The local Lorenz anomaly comes only from diagrams with $d_{\tilde{\alpha}}, y^{\tilde{\alpha}}$ loops and for the sake of a one-loop analysis it is sufficient to look only at the kinetic term of $d_{\tilde{\alpha}}, y^{\tilde{\alpha}}$ which we write as

$$
\int d^{2} z d_{\tilde{\alpha}}\left(\delta_{\tilde{\gamma}}^{\tilde{\alpha}} \bar{\partial}+\Omega_{\tilde{\gamma}}^{\tilde{\alpha}}\right) y^{\tilde{\gamma}}
$$


where $\Omega \equiv-\bar{\Pi}^{B}\left(\omega_{B \tilde{\gamma}}{ }^{\tilde{\alpha}}+w(\tilde{\gamma}) \Gamma_{B} \delta_{\tilde{\gamma}}^{\tilde{\alpha}}\right)$ contains the spin and $U(1)$ connection and depends only on the background fields. Local Lorentz and $U(1)$ transformations act on $d_{\tilde{\alpha}}, y^{\tilde{\alpha}}, \Omega_{\tilde{\gamma}}^{\tilde{\alpha}}$ as follows

$$
\begin{aligned}
\delta y^{\tilde{\beta}} & =\Lambda_{\tilde{\alpha}}^{\tilde{\alpha}} y^{\tilde{\alpha}} \\
\delta d_{\tilde{\alpha}} & =-d_{\tilde{\beta}} \Lambda_{\tilde{\alpha}}^{\tilde{\alpha}} \\
\delta \Omega_{\tilde{\gamma}}^{\tilde{\alpha}} & =\Lambda^{\tilde{\alpha}}{ }_{\tilde{\beta}} \Omega_{\tilde{\gamma}}^{\tilde{\beta}}-\Omega_{\tilde{\beta}}^{\tilde{\alpha}} \Lambda_{\tilde{\gamma}}^{\tilde{\beta}}-\bar{\partial} \Lambda^{\tilde{\alpha}}
\end{aligned}
$$

where $\Lambda$ is the parameter of the local Lorentz and $U(1)$ transformation that depends only on the background fields. The full Lorentz and $U(1)$ transformation rule for $d_{\tilde{\alpha}}$ may involve further $y^{A}$-dependent terms, depending on the choice of background expansion, but these do not contribute at one loop.

If we define an effective action by

$$
e^{-\frac{1}{\alpha^{\prime}} \Gamma[\Omega]}=\int D d D y e^{-\frac{1}{\alpha^{\prime}} \int d^{2} z d_{\tilde{\alpha}}\left(\delta_{\tilde{\gamma}} \tilde{\bar{\partial}} \bar{\partial}+\Omega_{\tilde{\gamma}}^{\tilde{\alpha}}\right) y^{\tilde{\alpha}}}
$$

one obtains for its anomalous variation

$$
\delta \Gamma[\Omega]=-\alpha^{\prime} \int d^{2} z \partial \Lambda^{\tilde{\alpha}}{ }_{\tilde{\gamma}} \Omega_{\tilde{\alpha}}^{\tilde{\gamma}} .
$$

To obtain this result one can e.g. use a point splitting regularization as in [35]. The full result for $\Gamma[\Omega]$ is proportional to $\Gamma_{W Z W}[g]$, the Wess-Zumino-Witten action, where the group valued field $g$ is related to $\Omega$ through $\Omega=g^{-1} \bar{\partial} g$, and is therefore a non-local functional of $\Omega$. One easily checks this correctly reproduces (4.54).

Our next task is to examine whether or not we can cancel (4.54) by a suitable transformation of the vielbein and the antisymmetric tensor. The relevant equation reads

$$
\delta\left(-\int d^{2} z\left(\frac{1}{2} \Pi^{a} \bar{\Pi}^{a}+D_{\tilde{\alpha}} \bar{\Pi}^{\tilde{\alpha}}+\frac{1}{2} \bar{\Pi}^{A} \Pi^{B} B_{B A}\right)\right)+\alpha^{\prime} \int d^{2} z \partial \Lambda^{\tilde{\alpha}} \Omega_{\tilde{\alpha}}^{\tilde{\gamma}}=0 .
$$

We will assume that the background fields are on-shell, in particular that $\bar{\Pi}^{\tilde{\alpha}}=0$. Furthermore we use $\Omega_{\tilde{\alpha}}^{\tilde{\gamma}}=-\bar{\Pi}^{A} \Omega_{A} \tilde{\tilde{\gamma}}$ and will abbreviate $\partial_{B} \Lambda_{\tilde{\alpha}}^{\tilde{\gamma}}\left(\omega_{A \tilde{\alpha}} \tilde{\gamma}+w(\tilde{\gamma}) \Gamma_{A} \delta_{\tilde{\alpha}} \tilde{\gamma}\right)$ by $\operatorname{Tr}\left(\partial_{B} \Lambda \hat{\omega}_{A}\right)$. Then we find from (4.55) that

$$
\delta \Pi^{A}=\Pi^{B} X_{B}{ }^{A}, \quad \delta \bar{\Pi}^{A}=\bar{\Pi}^{B} X_{B}{ }^{A}
$$

with

$$
\begin{aligned}
& X_{a b}=-\frac{\alpha^{\prime}}{2} \operatorname{Tr}\left(\partial_{b} \Lambda \hat{\omega}_{a}+\partial_{a} \Lambda \Omega_{b}\right) \\
& X_{\tilde{\alpha} b}=-\alpha^{\prime} \operatorname{Tr}\left(\partial_{\tilde{\alpha}} \Lambda \hat{\omega}_{b}+\partial_{b} \Lambda \Omega_{\tilde{\alpha}}\right) .
\end{aligned}
$$

In order that $\delta \bar{\Pi}^{\tilde{\alpha}}=0$, one has to choose $X_{a \tilde{\beta}}=0$, but $X_{\tilde{\alpha} \tilde{\beta}}$ can still be chosen arbitrarily. For the variation of the antisymmetric tensor we find

$$
\delta B_{B A}=-\alpha^{\prime} \operatorname{Tr}\left(\partial_{B} \hat{\omega}_{A}-(-1)^{A B} \partial_{A} \Lambda \hat{\omega}_{B}\right)-X_{B}{ }^{S} B_{S A}+(-1)^{A B} X_{A}{ }^{S} B_{S B},
$$

and with these variations (1.55) is indeed satisfied. 
What is the interpretation of these results? From the variations of the vielbein, we find that the metric $G_{M N}$ varies in exactly the same way as $-\frac{\alpha^{\prime}}{2} \operatorname{Tr}\left(\hat{\omega}_{M} \hat{\omega}_{N}\right)$. So instead of introducing new variations of the vielbein, we could also have redefined the metric as $G_{M N} \rightarrow G_{M N}-\frac{\alpha^{\prime}}{2} \operatorname{Tr}\left(\hat{\omega}_{M} \hat{\omega}_{N}\right)$, which is the same as adding a local counterterm to the world-sheet action. We have done an explicit calculation of the one-loop OPE's without neglecting the explicit $U(1)$ connections, and we found $U(1)$ noninvariant terms. All $U(1)$ noninvariant terms could be cancelled by a counterterm in the action of the form $\int d^{2} z \Gamma^{A} \Gamma^{B} \Pi_{B} \bar{\Pi}_{A}$, in perfect agreement with the general anomaly analysis presented above.

The variation of the antisymmetric tensor field cannot be canceled by a local counterterm. However, in the background field expansion all terms containing at least two quantum fields contain only the field strength $H$, and there is a unique local term one can add to $H$ in order to make it into a covariant quantity under the modified transformation rule of $B$,

$$
H_{M N P} \rightarrow H_{M N P}-\frac{\alpha^{\prime}}{2} \operatorname{Tr}\left(\hat{\omega}_{[M} \partial_{N} \hat{\omega}_{P\}}+\frac{2}{3} \hat{\omega}_{[M} \hat{\omega}_{N} \hat{\omega}_{P\}}\right) .
$$

In other words, one should add a super Chern-Simons three-form [36] to $H$ in order to make it covariant. We know that with the modified transformation rules discussed above, the Lorentz and $U(1)$ anomalies cancel, and therefore the result obtained from a perturbative calculation that takes all the spin and $U(1)$ connections properly into account, can also be obtained in a simpler way by first ignoring all the spin and $U(1)$ connections and by subsequently performing the substitution (4.59) in the final answer. In our computation we have ignored the spin and $U(1)$ connections, but we have only done a one-loop calculation and the shift in (4.59) would therefore only affect two-loop results. Altogether this shows that if we would have taken the spin and $U(1)$ connections into account, we would have been able to get rid of all non-covariant terms by means of suitable local counterterms, and the final field equations would still come out the same, and we verified this explicitly for the $U(1)$ connections, as mentioned above.

Similarly, the inclusion of gauge field from the internal six dimensional space or the heterotic fermions would give rise to a Chern-Simons term for the gauge fields in $H$, and these Chern-Simons terms play a crucial role in the anomaly cancelation through the Green-Schwarz mechanism.

\subsection{Standard $\beta$-function calculations}

In this section we briefly examine the relation between the diagrammatic results and a 'standard' beta-function calculation. In a standard beta-function calculation (see e.g. [12], [37, and for the Green-Schwarz string [38, 39]), one couples the sigma model to a world-sheet metric and then continues the theory to $2+2 \epsilon$ dimensions. If the $2+2 \epsilon$ dimensional metric is purely conformal, $g_{\alpha \beta} \sim e^{\sigma} \delta_{\alpha \beta}$, then the action picks up an overall factor $e^{\epsilon \sigma}$. In addition, the Fradkin-Tseytlin term $\int d^{2} z \sqrt{g} R(\phi+\bar{\phi})$ becomes proportional to $\int d^{2} z(\phi+\bar{\phi}) \partial \bar{\partial} \sigma$, and it explicitly breaks conformal invariance. This is not a problem, because the Fradkin-Tseytlin term is of higher order in $\alpha^{\prime}$, so that classically the sigma model is still conformally invariant. If we now vary the action with respect to $\sigma$, we find that $\delta S / \delta \sigma \sim \epsilon \mathcal{L}+\alpha^{\prime} c \partial \bar{\partial}(\phi+\bar{\phi})$, where $\mathcal{L}$ is the Lagrangian density, $S=\int d^{2} z \mathcal{L}$, and $c$ is some constant. If the theory is UV divergent at one-loop, it should be renormalized with 
a counterterm $\frac{\alpha^{\prime}}{\epsilon} \mathcal{L}_{\text {div }}$. Including such a counterterm, the condition for the theory to be conformally invariant at one-loop becomes $\mathcal{L}_{\text {div }} \sim \partial \bar{\partial}(\phi+\bar{\phi})$. The only one-loop diagrams that are UV divergent are those of the type of diagram (41), with the cross indicating a vertex from the action. If we denote the relevant vertices in (3.24) by $\int d^{2} z y^{A} y^{B} C_{B A}^{(2)}$, we find a UV divergent contribution $\frac{1}{\epsilon} C_{a a}^{(2)}$. Thus, conformal invariance of the sigma model requires $C_{a a}^{(2)} \sim \partial \bar{\partial}(\phi+\bar{\phi})$. Using the equations of motion of the background fields and the explicit form of the vertices $C_{B A}^{(2)}$ in (3.24), we find that this reproduces exactly the equations (4.28)-(4.30). Although this derivation is much simpler than the diagrammatic one in the previous sections, it is not clear to us whether these beta-function calculations would also be sufficient to guarantee the full $N=2$ superconformal invariance of the theory. As this example illustrates, the standard beta-function calculation reproduces precisely the equations of motion that are implied by requiring the presence of a Virasoro algebra only, and it may be that at higher loop this is a weaker condition than requiring a full $N=2$ invariance. Nevertheless, it would certainly be a useful exercise to compute the two-loop beta-functions of the sigma model, as these do provide necessary conditions for $N=2$ superconformal invariance. If the number of two-loop equations thus obtained would match the number of one-loop field equations, this would be a strong indication that one did in fact find the complete set of equations up to two-loop. These could then be used to find the $\alpha^{\prime}$ corrections to the supergravity action, which is discussed in the next section.

An alternative approach would be to first formulate the sigma model as a sigma model for a $(2,0)$ super world-sheet, and to then do a manifestly supersymmetric beta-function calculation (see e.g. 40]). This would automatically guarantee the full $N=(2,0)$ superconformal invariance. Unfortunately, no such super world-sheet formulation if the sigma model is known to us.

\section{The supergravity action}

In this section we derive the low energy effective action. The fields that appear in the theory are the vierbein $E_{M}^{A}$ and the dilaton complex chiral superfield $\phi$. The antisymmetric tensor $B_{A B}$ is not an indepent field, as it is expressed purely in terms of the vielbein by means of the tree-level constraints. We will find it convenient to define $\Phi=\exp \phi$ and $\bar{\Phi}=\exp \bar{\phi}$, so that $\Phi$ and $\bar{\Phi}$ have multiplicative $U(1)$ charge.

We have already seen that the field content of the low-energy theory is that of ' 16 - 16 supergravity' 41]. This supergravity can be expressed as a non-minimal supergravity with an additional constraint (such that the number of off-shell components of the nonminimal supergravity is reduced from 40 to 32). Solving for this constraint one sees that '16-16 supergravity' may be either old-minimal supergravity coupled to a tensor multiplet or a formulation of new-minimal supergravity coupled to a chiral multiplet 42. (Now the counting of the number of off-shell components yields $24+8 ; 24$ being the number of supergravity components and 8 the number of tensor or chiral multiplet components). The action that describes this non-minimal supergravity theory is given by 42

$$
S=\int E^{-1} G^{3 n+1}(\Phi \bar{\Phi})^{-2 n}
$$


where $G$ is a real linear multiplet and $\Phi$ is a chiral multiplet (the $\Phi$ in (5.1) is equal to $\left(\Phi^{\prime}\right)^{3 / 2}$, where $\Phi^{\prime}$ is the chiral multiplet that appears in 442; this follows from the $U(1)$ charge of our $\Phi$ or equivalently from its conformal weight (which is $3 / 2)$ ). Now in (5.1) either $G$ or $\Phi$ is a compensator field. Which one of the two is the compensator depends on the sign of the kinetic term of the scalar in the linear and chiral multiplet. If $G$ is a compensator we are dealing with a new-minimal theory, whereas in case $\Phi$ is the compensator the theory is old minimal. To determine the sign let us go to the gauge $\Phi=1$ and then shift $G \rightarrow G+1$. The factor 1 gives the usual supergravity action. The term quadratic in $G$ has coefficient $3 n(3 n+1)$. If this coefficient is positive then the kinetic term for the scalar in the tensor multiplet has the correct sign and $\Phi$ is the compensator.

The constraints that the supergravity algebra satisfies imply that we are in the "string gauge" $G=1$. In this gauge the field content of the tensor multiplet has been moved to the supergravity fields. The antisymmetric tensor that couples in the sigma model is sitting in the supergravity multiplet and not in the tensor multiplet. This follows from the sigma-model constraints

$$
T_{A B c}+(-1)^{A B} 2 H_{A B c}=0 .
$$

In addition, this relation implies

$$
H_{\alpha \dot{\beta} c}=-i C_{\alpha \gamma} C_{\dot{\beta} \dot{\gamma}}
$$

In rigid superspace the Bianchi identities for the tensor mupliplet give

$$
H_{\alpha \dot{\beta} c}=-i G C_{\alpha \gamma} C_{\dot{\beta} \dot{\gamma}}
$$

So we indeed get $G=1$.

The action (5.1) in the string gauge takes the form

$$
S=\int d^{4} x d^{4} \theta E^{-1}(\Phi \bar{\Phi})^{-2 n}
$$

We emphasize that the fact that one can gauge away $G$ does not mean that $G$ is a compensating multiplet. It is only the signs of the kinetic terms that determine which multiplet is the physical one, and which is the compensator.

To derive the field equations we will use a method developed by Wess and Zumino [43]. We define $\Delta_{A}{ }^{B}=\delta E_{A}{ }^{M} E_{M}{ }^{B}$. Then using

$$
\delta E^{-1}=-E^{-1}(-1)^{A} \Delta_{A}^{A} .
$$

the variation of the action is given by

$$
\delta S=\int E^{-1}\left[-(-1)^{A} \Delta_{A}{ }^{A}-2 n(\delta \phi+\delta \bar{\phi})\right](\Phi \bar{\Phi})^{-2 n} .
$$

However, $\Delta_{A}{ }^{A}, \delta \phi$ and $\delta \bar{\phi}$ are not unconstrained superfields. The variations of the superfields should be along the constrained surface defined by the original constraints. This imposes constraints on the variations. In other words, given $\nabla_{A}$ that satisfies (3.54)(3.57), $\nabla_{A}+\delta \nabla_{A}$, where

$$
\delta \nabla_{A}=\Delta_{A}^{B} \nabla_{B}+\delta \omega_{A \tilde{\beta}} \tilde{\gamma} M_{\tilde{\gamma}}^{\tilde{\beta}}+\delta \Gamma_{A} Y,
$$


should satisfy the same supergravity algebra to first order in the variations. We proceed to solve the constraints by expressing the variations in terms of prepotentials.

For the purpose of obtaining the field equations one need not solve for all the variations. All that we need is to express $\Delta_{a}{ }^{a}, \Delta_{\tilde{\alpha}}{ }^{C}, \Gamma_{\tilde{\alpha}}$ in terms of uncontrained superfields. The need for $\Delta_{\dot{\alpha}}{ }^{C}, \Gamma_{\dot{\alpha}}$ follows from the chirality of $\Phi$. The variation of $\Phi$ should respect its chirality

$$
0=\delta\left(\nabla_{\dot{\alpha}} \Phi\right)=\Delta_{\dot{\alpha}}^{C} \nabla_{C} \Phi-\frac{1}{2} \delta \Gamma_{\dot{\alpha}} \Phi+\nabla_{\dot{\alpha}}(\delta \Phi),
$$

and similarly for $\Delta_{\alpha}{ }^{C}, \Gamma_{\alpha}$.

We start by solving (3.54) (from now on "solving" means "solving at the linearized level"). It is rather easy to solve all equation that arise. The results are

$$
\begin{aligned}
\Delta_{\alpha}{ }^{\beta}= & \nabla_{\alpha} \psi^{\beta}-\psi_{\alpha}{ }^{\beta}-\frac{1}{2} C_{\alpha}{ }^{\beta} \psi \\
\Delta_{\alpha}{ }^{\dot{\beta}}= & \nabla_{\alpha} \bar{M}^{\dot{\beta}} \\
\Delta_{\alpha}{ }^{b}= & 2 i C_{\alpha}^{\beta} \bar{M}^{\dot{\beta}}+\nabla_{\alpha} L^{b} \\
\delta \Gamma_{\alpha}= & \nabla_{\alpha} \psi-4 i H_{\dot{\beta} \alpha} \bar{M}^{\dot{\beta}}+2 \nabla_{\beta} H_{\dot{\beta} \alpha} L^{b} \\
\delta \omega_{\alpha \gamma}{ }^{\delta}= & \left.\nabla_{\alpha} \psi_{\gamma}^{\delta}+2 i \bar{M}^{\dot{\gamma}} H_{\dot{\gamma}(\gamma \mid} C_{\left.\alpha\right|^{\delta}}{ }^{\delta}-L^{e} \nabla_{\epsilon} H_{\dot{\epsilon}(\gamma \mid} C_{\alpha \mid} \delta\right) \\
\delta \omega_{\alpha \dot{\gamma}} \dot{\delta}= & \nabla_{\alpha} \bar{\phi}_{\dot{\gamma}}^{\dot{\delta}}-2 i \bar{M}^{(\dot{\delta}} H_{\dot{\gamma}) \alpha}-2 i L_{\alpha \dot{\epsilon}} \bar{W}_{\dot{\gamma}}^{\dot{\delta}} \dot{\delta} \\
& \quad+\frac{1}{6} L_{\alpha(\dot{\gamma} \mid} \nabla^{\gamma \mid} H_{\gamma}^{\dot{\delta})}+\frac{1}{2}\left(L_{\dot{\gamma}}^{\epsilon} \nabla_{(\alpha \mid} H_{\mid \epsilon)}^{\dot{\delta}}+\dot{\gamma} \leftrightarrow \dot{\delta}\right)
\end{aligned}
$$

where $\psi^{\alpha}, \psi_{\alpha}{ }^{\beta}, \psi, \bar{M}^{\dot{\alpha}}, L^{a}, \bar{\phi}_{\dot{\gamma}}^{\dot{\delta}}$ are Lorentz irreducible superfields (e.g. $\psi_{\alpha}^{\alpha}=0$ ). At this point they are unconstrained but they will be constrained when we will impose the rest of the supergravity algebra. Notice that we have also solved for the spin connections. We did this because they will be needed in the subsequent analysis. The complex conjugate equations are obtained from the above by just interchanging dotted with undotted indices. Note, however, that since $\delta \Gamma_{\alpha} \rightarrow-\delta \Gamma_{\dot{\alpha}}$ (which follows from $Y^{\dagger}=-Y$, see Appendix A) we have $\psi \rightarrow-\bar{\psi}$.

Next, the torsion part of (3.55) implies

$$
\begin{aligned}
\Delta_{a}{ }^{\beta} & =\frac{i}{2}\left[\nabla_{(\alpha} \Delta_{\dot{\alpha})}{ }^{\beta}+\delta \omega_{\dot{\alpha} \alpha}{ }^{\beta}+\frac{1}{2} \delta \Gamma_{\dot{\alpha}} C_{\alpha}{ }^{\beta}\right], \\
\Delta_{a}{ }^{b} & =\frac{i}{2}\left[\nabla_{(\alpha} \Delta_{\dot{\alpha})}{ }^{b}-2 i \Delta_{\alpha}{ }^{\beta} C_{\dot{\alpha}}^{\dot{\beta}}-2 i \Delta_{\dot{\alpha}}{ }^{\dot{\beta}} C_{\alpha}{ }^{\beta}\right],
\end{aligned}
$$

From the curvature and $U(1)$ parts of (3.55) one can determine $\delta H_{\dot{\alpha} \alpha}, \delta \omega_{a \tilde{\beta}} \tilde{\gamma}, \delta \Gamma_{a}$. However, since we do not need these variations for the derivation of the field equations we will not solve the corresponding equations.

The commutator in (3.56) does not have any torsion part. This imposes certain constraints on the variations. Explicitly, we get that the following combinations should vanish

$$
-\nabla_{b} \Delta_{\alpha}^{\beta}+\Delta_{\alpha}{ }^{c} T_{c b}^{\gamma}+\nabla_{\alpha} \Delta_{b}^{\gamma}-\delta \omega_{b \alpha}^{\gamma}-\frac{1}{2} \delta \Gamma_{b} C_{\alpha}^{\gamma}=0
$$




$$
\begin{aligned}
& -\nabla_{b} \Delta_{\alpha}{ }^{\dot{\gamma}}+\Delta_{\alpha}{ }^{c} T_{c b}{ }^{\dot{\gamma}}+\nabla_{\alpha} \Delta_{b}^{\dot{\gamma}}=0 \\
& -\nabla_{b} \Delta_{\alpha}{ }^{c}+\Delta_{\alpha}{ }^{d} T_{d b}{ }^{c}+\delta \omega_{\alpha \beta}{ }^{\gamma} C_{\dot{\beta}}^{\dot{\gamma}}+\delta \omega_{\alpha \dot{\beta}}{ }^{\dot{\gamma}} C_{\beta}^{\gamma}+\nabla_{\alpha} \Delta_{b}^{c}+2 i \Delta_{b}{ }^{\dot{\gamma}} C_{\beta}^{\gamma}=0
\end{aligned}
$$

The first equation will constrain the prepotentials for $\delta \omega_{a \tilde{\beta}} \tilde{\gamma}, \delta \Gamma_{a}$. Since we are not interested in them we will not discuss further this equation. Equations (5.13) and (5.14) constrain the prepotentials $\psi^{\alpha}, \psi_{\alpha}^{\beta}, \psi, \bar{M}^{\dot{\alpha}}, L^{a}, \bar{\phi}_{\dot{\gamma}}{ }^{\dot{\delta}}$ as we now show.

Inserting into (5.13) the results from (5.10) and (5.11) and after some rather tedious algebra we get that certain combinations of the prepotentials are linear superfields. We solve these constraints to get

$$
\begin{aligned}
& \nabla^{\dot{\alpha}}\left(\bar{\psi}_{\dot{\alpha}}-\bar{M}_{\dot{\alpha}}\right)+\psi-\bar{\psi}=\nabla^{\alpha} \Lambda_{\alpha} \\
& \nabla_{(\dot{\alpha}}\left(\bar{\psi}_{\dot{\beta})}-\bar{M}_{\dot{\beta})}\right)+2\left(\bar{\phi}_{\dot{\alpha} \dot{\beta}}-\bar{\psi}_{\dot{\alpha} \dot{\beta}}\right)=\nabla^{\alpha} K_{\alpha \dot{\alpha} \dot{\beta}}
\end{aligned}
$$

where $\Lambda_{\alpha}, K_{\alpha \dot{\alpha} \dot{\beta}}$ are (at this point) unconstrained superfields. The calculation involves a rather delicate cancellation among all terms that are not linear in the fields or in the variations. One may anticipate such a cancellation since the solution of the full non-linear Bianchi's involves $H_{\dot{\alpha} \alpha}$ and $W_{\alpha \beta \gamma}$ only linearly.

Procceding in a similar way we get from (5.14) (again after tedious algebra and many cancellations)

$$
\begin{aligned}
& \frac{i}{2} \nabla_{\dot{\alpha}}\left(L^{a}-\bar{L}^{a}\right)-\Lambda^{\alpha}+2\left(M^{\alpha}-\psi^{\alpha}\right)=\nabla^{\beta} \theta_{\beta}{ }^{\alpha}+\nabla^{\alpha} \theta \\
& K_{\dot{\beta}}^{\alpha} \dot{\gamma}+\frac{i}{2} \nabla_{(\dot{\beta} \mid}\left(L^{\alpha \mid \dot{\gamma})}-\bar{L}^{\alpha \mid \dot{\gamma})}\right)=\nabla^{\beta} \Sigma_{\beta}{ }^{\alpha} \dot{\beta}{ }^{\dot{\gamma}}+\nabla^{\alpha} \Sigma_{\dot{\beta}}{ }^{\dot{\gamma}}
\end{aligned}
$$

where $\theta_{\beta}{ }^{\alpha}, \Sigma_{\beta}{ }^{\alpha} \dot{\beta} \dot{\gamma}, \Sigma_{\dot{\beta}} \dot{\gamma}$ are Lorentz irreducible.

We see that these equations only depend on certain differences of superfields. We define

$$
\hat{\psi}=\psi-\bar{\psi} ; \quad \hat{\phi}_{\alpha \beta}=\phi_{\alpha \beta}-\psi_{\alpha \beta} ; \quad \hat{L}^{a}=L^{a}-\bar{L}^{a} ; \quad \hat{M}^{\alpha}=M^{\alpha}-\psi^{\alpha} .
$$

In terms of these fields the equations (5.15), (5.17) and their complex conjugate read

$$
\begin{aligned}
& \nabla_{\dot{\alpha}} \overline{\hat{M}}^{\dot{\alpha}}+\hat{\psi}=\nabla^{\alpha} \Lambda_{\alpha} \\
& \nabla_{\alpha} \hat{M}^{\alpha}+\hat{\psi}=\nabla^{\dot{\alpha}} \bar{\Lambda}_{\dot{\alpha}} \\
& \frac{i}{2} \nabla_{\dot{\alpha}} \hat{L}^{a}-\Lambda^{\alpha}+2 \hat{M}^{\alpha}=\nabla^{\beta} \theta_{\beta}{ }^{\alpha}+\nabla^{\alpha} \theta \\
& -\frac{i}{2} \nabla_{\alpha} \hat{L}^{a}-\bar{\Lambda}^{\dot{\alpha}}+2 \overline{\hat{M}}^{\dot{\alpha}}=\nabla^{\dot{\beta}} \bar{\theta}_{\dot{\beta}}^{\dot{\alpha}}+\nabla^{\dot{\alpha}} \bar{\theta} \\
& -\nabla_{(\dot{\alpha}} \overline{\hat{M}}_{\dot{\beta})}+2 \overline{\hat{\phi}}_{\dot{\alpha} \dot{\beta}}=\nabla^{\alpha} K_{\alpha \dot{\alpha}}{ }^{\dot{\beta}} \\
& -\nabla_{(\alpha} \hat{M}_{\beta)}+2 \hat{\phi}_{\alpha \beta}=\nabla^{\dot{\alpha}} \bar{K}_{\dot{\alpha} \alpha}{ }^{\beta} \\
& K^{\alpha}{ }_{\dot{\beta}}^{\dot{\alpha}}+\frac{i}{2} \nabla_{(\dot{\beta} \mid} \hat{L}^{\alpha \mid \dot{\alpha})}=\nabla^{\beta} \Sigma_{\beta}{ }^{\alpha} \dot{\beta} \\
& \bar{K}^{\dot{\alpha}}{ }_{\beta}{ }^{\alpha}-\frac{i}{2} \nabla_{(\beta} \hat{L}^{\alpha) \dot{\alpha}}=\nabla^{\alpha} \Sigma_{\dot{\beta}}{ }^{\dot{\gamma}} \bar{\Sigma}_{\dot{\beta}} \dot{\alpha} \beta^{\gamma}+\nabla^{\dot{\alpha}} \bar{\Sigma}_{\beta}{ }^{\gamma} .
\end{aligned}
$$


The last four equations are irrelevant for the derivation of the field equations and, therefore, we will not deal with them any further. The first four equations constrain the superfields $\hat{\psi}, \hat{M}, \Lambda^{\alpha}, \theta_{\alpha \beta}, \theta$. We now solve them in terms of new prepotentials (prepotentialsfor-prepotentials). Equations (5.21) and (5.22) can be used to solve for $\Lambda^{\alpha}$ and $\Lambda^{\dot{\alpha}}$. Eliminating $\hat{\psi}$ from (5.19) and (5.20) we get

$$
-\nabla^{\alpha} \hat{M}_{\alpha}+\nabla^{\dot{\alpha}} \overline{\hat{M}}_{\dot{\alpha}}=-\nabla^{\alpha}\left(\frac{i}{2} \nabla^{\dot{\alpha}} \hat{L}_{a}+\nabla_{\alpha} \theta\right)+\nabla^{\dot{\alpha}}\left(-\frac{i}{2} \nabla^{\alpha} \hat{L}_{a}+\nabla_{\dot{\alpha}} \bar{\theta}\right)
$$

The general solution of this equation is a particular solution $\hat{M}_{\alpha}^{1}$ plus the most general solution $\hat{M}_{\alpha}^{0}$ of the homogeneous equation. It is very easy to find a particular solution

$$
\hat{M}_{\alpha}^{1}=\frac{i}{2} \nabla^{\dot{\alpha}} L_{a}+\nabla_{\alpha} \theta
$$

The general solution of the homogeneous equation is given by

$$
\hat{M}_{\alpha}^{0}=\bar{\nabla}^{2} \nabla_{\alpha} V+\nabla^{\beta} \chi_{\beta \alpha}+\nabla^{2} \chi_{\alpha} .
$$

where $V$ is a real unconstrained superfield and $\chi_{\alpha \beta}, \chi$ are Lorentz irreducible unconstrained complex supefields. To prove the last statement we rewrite the equation as

$$
\nabla^{\alpha} \hat{M}_{\alpha}=\nabla^{\dot{\alpha}} \overline{\hat{M}}_{\dot{\alpha}}
$$

The general solution of this equation is a particular solution plus a general element in the kernel of $\nabla_{\alpha}$. The last two terms in (5.29) are clearly the most general elements in the kernel of $\nabla^{\alpha}$. It remains to show that the first term in the right hand side of (5.29) is a particular solution of (5.30). This follows from the identity

$$
\nabla^{\alpha} \bar{\nabla}^{2} \nabla_{\alpha} V=\nabla^{\dot{\alpha}} \nabla^{2} \nabla_{\dot{\alpha}} V
$$

which can be proven by direct computation. We have, thus, shown that

$$
\hat{M}_{\alpha}=\frac{i}{2} \nabla^{\dot{\alpha}} L_{a}+\nabla_{\alpha} \theta+\bar{\nabla}^{2} \nabla_{\alpha} V+\nabla^{\beta} \chi_{\beta \alpha}+\nabla^{2} \chi_{\alpha}
$$

Furthermore,

$$
\begin{aligned}
\Lambda^{\alpha} & =-\frac{i}{2} \nabla_{\dot{\alpha}} \hat{L}^{a}+2 \bar{\nabla}^{2} \nabla^{\alpha} V+\nabla^{\alpha} \theta+\nabla^{\beta}\left(2 \chi_{\beta}{ }^{\alpha}-\theta_{\beta}{ }^{\alpha}\right)+2 \nabla^{2} \chi^{\alpha}, \\
\hat{\psi} & =\frac{i}{2}\left[\nabla_{\alpha}, \nabla_{\dot{\alpha}}\right] L^{a}+3 \nabla^{\alpha} \bar{\nabla}^{2} \nabla_{\alpha} V+\nabla^{2} \theta+\bar{\nabla}^{2} \bar{\theta}
\end{aligned}
$$

Next, we are going to express the variation of the dilaton superfield in terms of unconstrained superfields. We need to solve equation (5.9). This is rather straightforward. The result is

$$
\delta \Phi=\nabla^{2} U-M^{\alpha} \nabla_{\alpha} \Phi-\bar{L}^{b} \nabla_{b} \Phi+\frac{1}{2} \bar{\psi} \Phi
$$

where $U$ is a complex unconstrained superfield.

We are now almost ready to derive the field equations. We first need one more fact, namely that our supergravity algebra allows naive partial integrations. This follows from the identity

$$
E^{-1} \overleftarrow{\nabla}_{A}=-E^{-1}(-1)^{B} T_{A B}^{B}
$$


The proof of this identity can be found in 444 p. 282, and it will not be repeated here. Since in our supergravity algebra $(-1)^{B} T_{A B}{ }^{B}=0$ naive partial integrations are allowed.

The easiest equation to derive is the dilaton field equation. Integrating by part the derivatives from $U$ and using the fact that $\bar{\Phi}$ is anti-chiral we get

$$
\nabla^{2} \Phi=0
$$

or in terms of $\phi$

$$
\nabla^{2} \phi+\nabla^{\alpha} \phi \nabla_{\alpha} \phi=0
$$

We now move on to derive the remaining equations. Direct computation gives

$$
\begin{aligned}
-(-1)^{A} \Delta_{A}{ }^{A}= & 2\left[\nabla_{\alpha}\left(M^{\alpha}-\psi^{\alpha}\right)+\nabla_{\dot{\alpha}}\left(\bar{M}^{\dot{\alpha}}-\bar{\psi}^{\dot{\alpha}}\right)\right]+\psi-\bar{\psi} \\
& -\frac{i}{2}\left(\nabla_{\alpha} \nabla_{\dot{\alpha}} \bar{L}^{a}+\nabla_{\dot{\alpha}} \nabla_{\alpha} L^{a}\right)+\nabla_{\alpha} \psi^{\alpha}+\nabla_{\dot{\alpha}} \bar{\psi}^{\dot{\alpha}}
\end{aligned}
$$

We see that some terms appear in the combinations of differences that we have already seen but not all of them. However, an interesting conspiracy happens when we take into account the dilaton terms. Integrating by parts the $M$-term in $\delta \phi$ the resulting $\nabla_{\alpha} M^{\alpha}$ combines with the bare $\nabla_{\alpha} \psi^{\alpha}$ in (5.38) to $\nabla_{\alpha} \hat{M}^{\alpha}$. Similarly, by integrating by parts the $L$ terms in $\delta \phi$ and $\delta \bar{\phi}$ we find that the $L$ terms also organize themselves into $\hat{L}^{a}$ (more precisely, we integrate by parts the term $-\frac{1}{2} \bar{L}^{b} \nabla_{b} \Phi$, the other half we keep as it is; similarly for the $L$ term in $\delta \bar{\phi}$ ). At this point we have

$$
\delta S=\int E^{-1}\left[\nabla_{\alpha} \hat{M}^{a}+\nabla_{\dot{\alpha}} \overline{\hat{M}}^{\dot{\alpha}}+(1+n) \hat{\psi}+\frac{i}{4}\left[\nabla_{\alpha}, \nabla_{\dot{\alpha}}\right] L^{a}-n L^{a} \nabla_{a}(\phi-\bar{\phi})\right](\Phi \Phi)^{-2 n}
$$

Inserting now (5.32), (5.33) we get

$$
\begin{aligned}
\delta S= & \int E^{-1}\left[\frac{i}{4}(2 n+1)\left[\nabla_{\alpha}, \nabla_{\dot{\alpha}}\right] \hat{L}^{a}-n \hat{L}^{a} \nabla_{a}(\phi-\bar{\phi})+\right. \\
& \left.+n\left(\nabla^{2} \theta+\bar{\nabla}^{2} \bar{\theta}\right)+(1+3 n) \nabla^{\alpha} \bar{\nabla}^{2} \nabla_{\alpha} V\right](\Phi \bar{\Phi})^{-2 n}
\end{aligned}
$$

Clearly, $\theta$ and $\bar{\theta}$ do not give any new equations. For $n=-1 / 2$ the first term in (5.40) drops out and we get the following field equation

$$
\nabla_{a}(\phi-\bar{\phi})=0,
$$

Clearly, this equation implies (4.46). Equation (4.46), however, is a slightly weaker condition than that given by (5.41). We think that it is very unlkely that it is possible to write down a different action that gives directly (4.46) as its field equation instead of just (5.41). It is clear though that in the class of theories described by an action of the form (5.5) the $n=-1 / 2$ theory is the only one that can be the low energy effective action of the heterotic superstring. Furthermore, we expect that a two-loop calculation will confirm the value $n=-1 / 2$.

For $n=-1 / 2$ (which is the $n=0, \tilde{n}=-1 / 2$ case in the language of [44) $3 n(3 n+1)$ is positive, and therefore, the low energy theory is old-minimal supergravity coupled to a tensor multiplet. The fact that the low energy effective action of the heterotic string is old-minimal supergravity was already predicted in [5] using string field theory 
arguments, and that it is in particular $n=-1 / 2$ '16-16 supergravity' in [6]. Properties of low energy effective actions similar to ours have been studied in [45, although the dilaton was incorrectly assumed to be part of $G$ rather than $\Phi$.

It remains to determine the field equation that follows from $V$. It turns out that this equation follows from the other two as we now describe. Integrating by parts the derivatives from $V$ and using that $\Phi$ is chiral and linear (see (5.36) ) we get

$$
\left(\nabla_{\alpha} \nabla_{\dot{\beta}} \nabla^{\dot{\beta}} \nabla^{\alpha} \Phi\right) \bar{\Phi}+2\left(\nabla_{\alpha} \nabla^{\dot{\beta}} \nabla^{\alpha} \Phi\right)\left(\nabla_{\dot{\beta}} \bar{\Phi}\right)+2\left(\nabla^{\dot{\beta}} \nabla^{\alpha} \Phi\right)\left(\nabla_{\alpha} \nabla_{\dot{\beta}} \bar{\Phi}\right)=0
$$

Adding to this equation its complex conjugate we get

$$
\nabla^{a} \phi \nabla_{a} \bar{\phi}-H^{a} H_{a}+\frac{i}{4}\left[\nabla_{\alpha}, \nabla_{\dot{\alpha}}\right] H^{\dot{\alpha} \alpha}=0
$$

To get this result one has to use the following two identities

$$
\begin{aligned}
& \nabla_{\alpha} \nabla^{\dot{\alpha}} \nabla^{\alpha} \phi=0 \\
& \frac{1}{2}\left(\nabla_{\alpha} H^{\dot{\alpha} \alpha} \nabla_{\dot{\alpha}} \bar{\phi}-\nabla_{\dot{\alpha}} H^{\dot{\alpha} \alpha} \nabla_{\alpha} \phi\right)-H^{\dot{\alpha} \alpha} \nabla_{\dot{\alpha}} \bar{\phi} \nabla_{\alpha} \phi=0 .
\end{aligned}
$$

that both follow from (5.41).

It is now relatively straightforward to prove that (5.43) follows from (5.36) and (5.41). Equation (5.41) can be rewritten as

$$
H_{\dot{\alpha} \alpha}=-\frac{i}{4}\left[\nabla_{\alpha}, \nabla_{\dot{\alpha}}\right](\phi+\bar{\phi})
$$

Acting with $\left[\nabla_{\alpha}, \nabla_{\dot{\alpha}}\right]$ one gets

$$
\left[\nabla_{\alpha}, \nabla_{\dot{\alpha}}\right] H^{\dot{\alpha} \alpha}=-i \nabla^{a} \nabla_{a}(\phi+\bar{\phi})
$$

Similarly by acting with $\nabla^{\dot{\alpha}} \nabla^{\alpha}$ on the dilaton field equation we get

$$
\nabla^{a} \nabla_{a}(\phi+\bar{\phi})+2 \nabla^{a} \phi \nabla_{a} \phi-2 H^{a} H_{a}-\frac{i}{2}\left[\nabla_{\alpha}, \nabla_{\dot{\alpha}}\right] H^{\dot{\alpha} \alpha}=0 .
$$

From (5.46) and (5.47) one immediately gets (5.43).

\section{Conclusions}

We have performed a one-loop computation to determine the low energy effective action of the heterotic superstring. This was done by first coupling the Berkovits string to a curved background and then requiring that it has an $N=2$ superconformal invariance at the one-loop level. The tree-level analysis gave a set of constraints that together with a maximal set of conventional constraints determined completely the supergravity algebra through the Bianchi identities.

Computing the OPE's of the superconformal algebra at the one-loop level led to extra terms in the right hand side of the OPE's. The extra holomorphic terms could

be cancelled by a particular and ill-understood choice of counterterms and background 

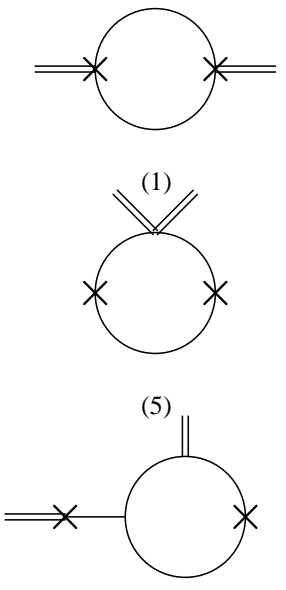

(9)

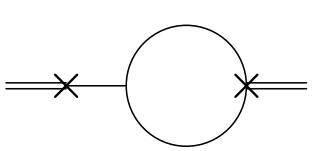

(13)
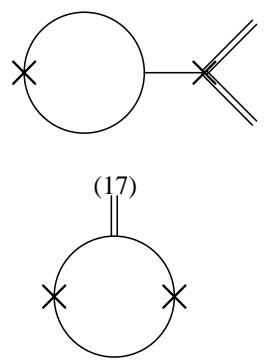

(21)

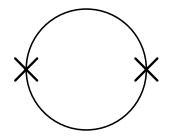

(25)

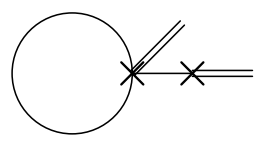

(29)

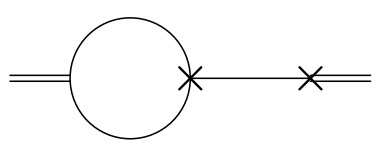

(33)

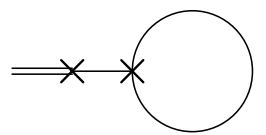

(37)

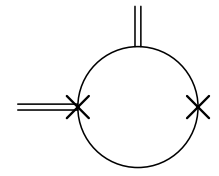

(2)

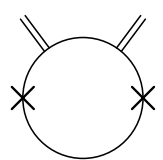

(6)
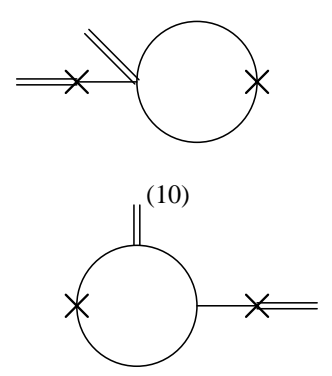

(14)

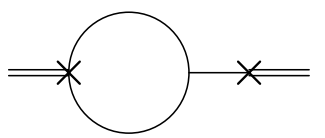

(18)

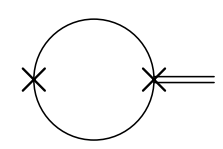

(22)

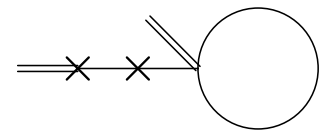

(26)

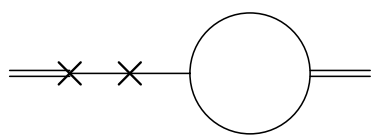

(30)

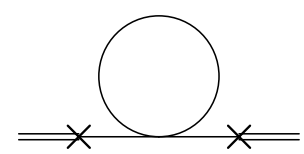

(34)

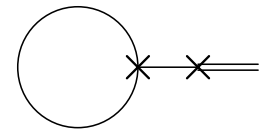

(38)

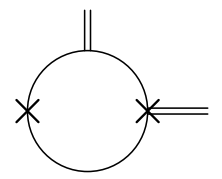

(3)

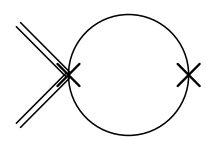

(7)

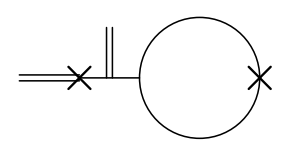

(11)

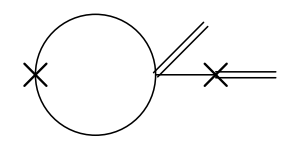

(15)

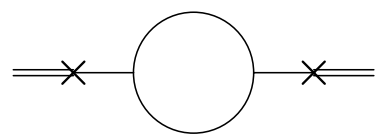

(19)

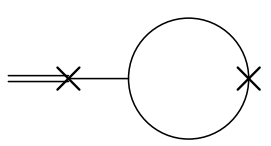

(23)

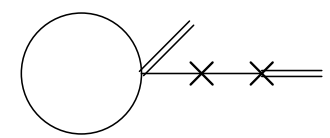

(27)

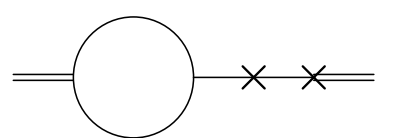

(31)

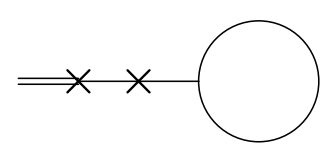

(35)
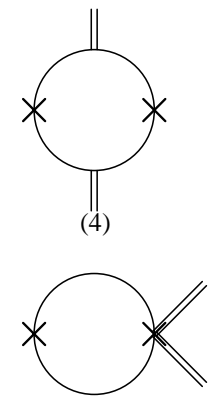

(8)

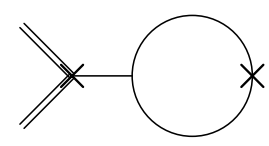

(12)

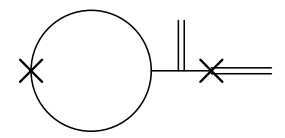

(16)

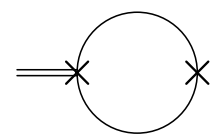

(20)

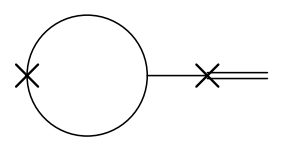

(24)

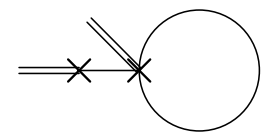

(28)

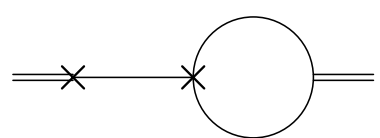

(32)

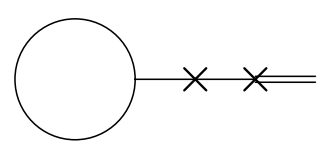

(36) 


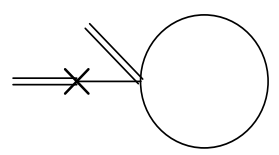

(39)

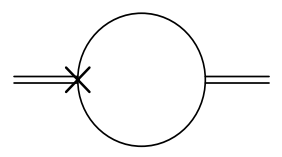

(43)

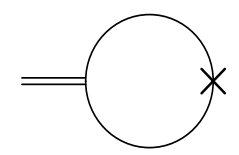

(47)

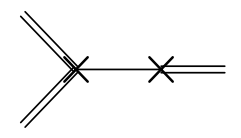

(51)

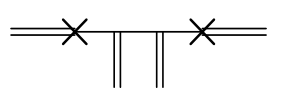

(55)

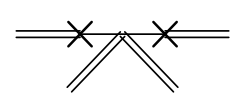

(59)

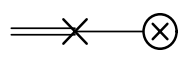

(63)

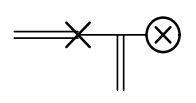

(67)

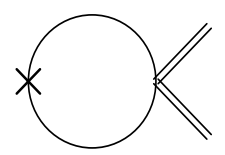

(40)

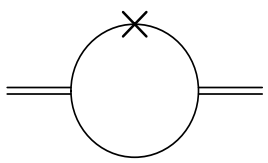

(44)

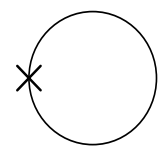

(48)

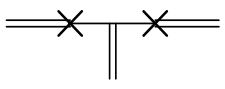

(52)

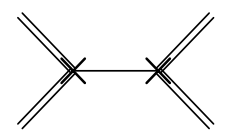

(56)

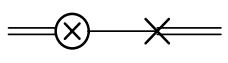

(60)

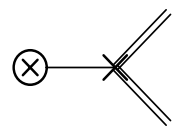

(64)

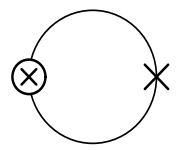

(68)

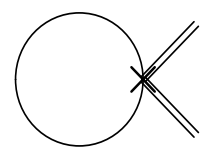

(41)

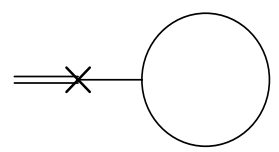

(45)

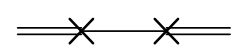

(49)

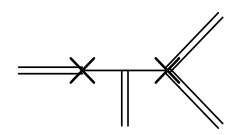

(53)

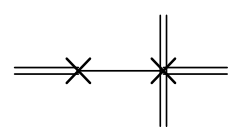

(57)

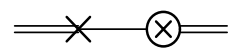

(61)

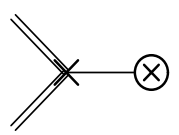

(65)

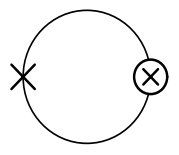

(69)

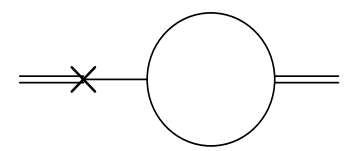

(42)

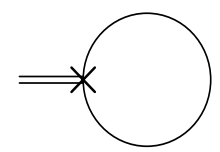

(46)

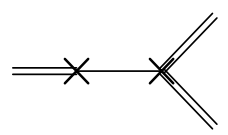

(50)

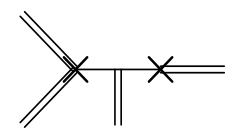

(54)

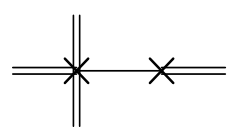

(58)

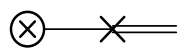

(62)

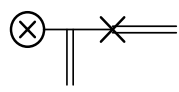

(66)

$\otimes$

(70)

Figure 1: A table of Feynman diagrams; crosses $(\times)$ indicate vertices from the nondilaton parts of $T, G$, and $\bar{G}$; encircled crosses $(\otimes)$ indicate vertices coming from their dilaton parts; double lines indicate background fields and single lines indicate quantum fields. Whenever the diagram is a contribution to $\langle A(z) B(w)\rangle$, the leftmost cross will always indicate a vertex from $A$, the rightmost cross a vertex from $B$. 
field expansions. The non-holomorphic terms led to the field equations. We found field equations only in the OPE's of $T$ with $G$ and $T$ with $T$. This is accordance with the strange $\alpha^{\prime}$ factors that appears in the definition of $G$ and $\bar{G}$. The one-loop calculation of $\langle G(z) \bar{G}(w)>$ yields only the tree-level part of $T$, and should therefore not contain any field equations (similarly for $\langle G(z) G(w)>,<\bar{G}(z) \bar{G}(w)>$ ). The calculation of $<T(z) T(w)>$ led to three equations. However, only one of those is an independent equation, namely

$$
\nabla_{\gamma} \nabla_{a}(\phi-\bar{\phi})=0
$$

The other two can be obtained by differentiating (6.1). Vanishing of the non-holomorphic part of $<T(z) G(w)>$ requires that precisely the same equation holds.

We then studied the problem of finding the supergravity action that has (6.1) as its field equation. Starting with a general form of the supergravity action (5.5) we obtained that only the $n=1 / 2$ case

$$
S=\int d^{4} x d^{4} \theta E^{-1} \Phi \bar{\Phi}
$$

is compatible with the string calculation. Hence, we conlude that this is the low energy effective action. This action describes old-minimal supergravity coupled to a tensor multiplet. Notice, however, that instead of equation (6.1) we find a slightly stronger field equation, namely

$$
\nabla_{a}(\phi-\bar{\phi})=0
$$

Another way to check that $n=1 / 2$ is consistent is to look at the precise coefficient of the dilaton term in the sigma model action, and to use that one the sphere $\frac{1}{4} \int d^{2} z R \sqrt{g}=-2$.

The action 6.2 leads to two more field equations

$$
\begin{aligned}
\nabla^{2} \phi+\nabla^{\alpha} \phi \nabla_{\alpha} \phi & =0 \\
\nabla^{a} \phi \nabla_{a} \bar{\phi}-H^{a} H_{a}+\frac{i}{4}\left[\nabla_{\alpha} \nabla_{\dot{\alpha}}\right] H^{\dot{\alpha} \alpha} & =0
\end{aligned}
$$

We saw a glimpse of the first one in the computation of $\langle G(z) G(w)>$, and the second is a consequence of the first one and (6.3). Both will probably follow from a two-loop calculation.

There are many different extensions and applications of our results. One extension is to include the compactification-dependent and the Yang-Mills background fields. This extension should be straightforward since all our methods are directly applicable. One may also try to extend our results to two loops. This is, however, a much more difficult task since the number of graphs one would need to compute grows enormously and, furthermore, one runs into problems of renormalization. It would be desirable to have a better understanding of the counterterms and different background field expansions for $d$ before starting such a two-loop calculation. At this moment, the easiest way to obtain two-loop information seems to be to compute the conventional beta-functions, as discussed in section 4.4, at two loops. Further generalizations include the case of the type II string. Since the sigma model treats Neveu-Schwarz fields on the same footing as Ramond fields, the sigma model might provide interesting information about the geometry of the still, especially in the case of the type IIB string, mysterious Ramond fields. It should also be possible to generalize the covariant sigma model description of the heterotic string to the case of $N=(1,2)$ heterotic strings. These string theories 
have recently attracted some attention because they may be of direct relevance to $M$ theory [47]. Another interesting possibility is to consider $D$-branes in an open type II version of the sigma model. Due to the simple nature of the Ramond fields, the Ramond charges carried by a $D$-brane should be clearly visible. In a somewhat different direction our results might shed light in the relation between target space supersymmetry and $T$-duality, and lead to new off-shell formulations for certain supergravity theories.

All in all, we believe that this world-sheet point of view of string theory should provide a fruitful arena for further investigations.

\section{Acknowledgements}

We would like to thank E. Sezgin, K. Stelle, and P. van Nieuwenhuizen for collaboration at the early stages of this work, and M. Roček and especially N. Berkovits and W. Siegel for very many helpful discussions during the various stages of this project. We would further like to thank G. Papadopoulos for a discussion on background field expansions, and M.B. Halpern for discussions on the Feynman rules we used. This work was supported in part by the National Science Foundation under grant PHY93-09888.

\section{A Conventions}

In this appendix we present the conventions used in this paper. We use a two component notation. Our conventions are the ones of 44. For completeness we summarize the relevant points here. Indices from the first part of the alphabet (Greek or Roman) are target space (or flat) indices and from the middle part of the alphabet are curved indices. Greek letters are reserved for spinor two components indices (for example, $\psi^{\alpha}$ is a two component spinor with $\alpha=+,-)$ and Roman ones for vector indices. Each vector index is equivalent to one undotted and one dotted index $\left(\phi^{a}=\phi^{\alpha \dot{\alpha}}\right)$. Capital indices denote both a spinor (dotted and undotted) and vector index, whereas a tilde on a Greek index denotes both a dotted and undotted spinor index. Indices are raised and lowered using an $s l_{2}$ invariant antisymmetric two dimensional matrix $C_{\alpha \beta}$. Since $C_{\alpha \beta}$ is antisymmetric, we have to specify how exactly we use it to raise and lower indices, and our convention is the so-called 'down-hill' rule from left to right for both the undotted and the dotted sector.

To illustrate the above we give couple of examples:

$$
\begin{aligned}
& \psi^{A} \chi_{A}=\psi^{a} \chi_{a}+\psi^{\tilde{\alpha}} \chi_{\tilde{\alpha}}=\psi^{a} \chi_{a}+\psi^{\alpha} \chi_{\alpha}+\psi^{\dot{\alpha}} \chi_{\dot{\alpha}} \\
& \psi^{\alpha} C_{\alpha \beta}=\psi_{\beta} ; \quad C^{\alpha \beta} \psi_{\beta}=\psi^{\alpha}
\end{aligned}
$$

In addition, we have the following identity

$$
C_{\alpha \beta} C^{\gamma \delta}=\delta_{[\alpha}^{\gamma} \delta_{\beta]}^{\delta}
$$

where the square brackets indicate antisymmetrization. Similarly, parenthesis indicate symmetrization, and square bracket from the left and parenthesis from the right indicate 
graded antisymmetrization. Our convention for (anti)-symmetrization is without any additional factors, i.e.

$$
A_{(\alpha} B_{\beta)}=A_{\alpha} B_{\beta}+A_{\beta} B_{\alpha} .
$$

Furthermore, indices between straight lines do not participate in (anti)-symmetrization.

From (A.3) we get

$$
\begin{aligned}
& C^{\alpha \beta} C_{\alpha \beta}=\delta_{\alpha}{ }^{\alpha}=2 \\
& C_{\alpha \beta} \chi_{\gamma}-C_{\alpha \gamma} \chi_{\beta}=-C_{\beta \gamma} \chi_{\alpha} \\
& \chi_{\alpha} \chi_{\beta}=-\frac{1}{2} C_{\alpha \beta} \chi^{\gamma} \chi_{\gamma}
\end{aligned}
$$

The last identity is an example of a Fierz identity. Finally, although we are not going to need it, we quote the explicit form of the $C$-matrices

$$
C_{\alpha \beta}=C^{\dot{\beta} \dot{\alpha}}=C_{\dot{\alpha} \dot{\beta}}=\sigma_{2}=\left(\begin{array}{cc}
0 & -i \\
i & 0
\end{array}\right),
$$

where $\sigma_{2}$ is the Pauli matrix.

Our conventions for covariant derivatives, torsion, curvature, etc. are as follows

$$
\begin{gathered}
\nabla_{A}=E_{A}{ }^{M} \partial_{M}+\omega_{A \beta}{ }^{\gamma} M_{\gamma}{ }^{\beta}+\omega_{A \dot{\beta}}{ }^{\dot{\gamma}} M_{\dot{\gamma}}^{\dot{\beta}}+\Gamma_{A} Y, \\
{\left[\nabla_{A}, \nabla_{B}\right\}=T_{A B}{ }^{C} \nabla_{C}+R_{A B \gamma}{ }^{\delta} M_{\delta}^{\gamma}+R_{A B \dot{\gamma}}{ }^{\dot{\delta}} M_{\dot{\delta}}^{\dot{\gamma}}+F_{A B} Y,}
\end{gathered}
$$

where $E_{A}{ }^{M}, \omega_{A \tilde{\beta}} \tilde{\gamma}$ and $\Gamma_{A}$ are the vielbein, the spin connection and the $U(1)$ connection, respectively. $T_{A B}{ }^{C}, R_{A B \tilde{\gamma}} \tilde{\delta}$ and $F_{A B}$ are the torsion, the curvature tensor and the $U(1)$ curvature, repectively. $M_{\tilde{\delta}}^{\tilde{\gamma}}$ are the generators of the Lorentz group. They are symmetric in $\alpha, \beta$ and they act as follows

$$
\left[\lambda_{\alpha}{ }^{\beta} M_{\beta}{ }^{\alpha}, s_{\gamma}\right]=\lambda_{\gamma}{ }^{\alpha} s_{\alpha},
$$

Finally, $Y$ is the antihermitian generator of $U(1)$. The $U(1)$ charge of the various fields is given in the main text. In addition, we sometimes use $w(A)$ to indicate the $U(1)$ weight of the index $A$, which is defined by $w(\alpha)=+\frac{1}{2}, w(\dot{\alpha})=-\frac{1}{2}$, and $w(a)=0$. Let us also give the explicit dependence of the torsion on the vierbein and the connections

$$
T_{A B}^{C}=E_{[A}^{M} \partial_{M} E_{B)}{ }^{N} E_{N}{ }^{C}+\omega_{[A B)}^{C}+w(C) \Gamma_{[A} \delta_{B)}{ }^{C} .
$$

Both the spin connection and the curvature are reducible with respect to the Lorentz group, in order to preserve (3.52). In particular, one has

$$
\begin{aligned}
\omega_{A b}{ }^{c} & =\omega_{A \beta}{ }^{\gamma} \delta_{\dot{\beta}}^{\dot{\gamma}}+\omega_{A \dot{\beta}} \dot{\gamma}^{\dot{\gamma}} \delta^{\gamma} \\
\omega_{A \beta}{ }^{c} & =\omega_{A \beta}{ }^{\dot{\gamma}}=0 \\
R_{A B c}{ }^{d} & =R_{A B \gamma}{ }^{\delta} \delta_{\dot{\gamma}}^{\dot{\delta}}+R_{A B \dot{\gamma}}{ }^{\dot{\delta}} \delta_{\gamma}{ }^{\delta} \\
R_{A B c}{ }^{\delta} & =R_{A B \gamma}{ }^{\gamma}=R_{A B \gamma}{ }^{\delta}=0 .
\end{aligned}
$$

Finally, we use a version of hermitian conjugation, which we denote by c.c., and which acts as follows on the various objects

$$
\begin{array}{r}
\left(\nabla_{\alpha}\right)^{\dagger}=\nabla_{\dot{\alpha}} ; \quad\left(C_{\alpha \beta}\right)^{\dagger}=C_{\dot{\alpha} \dot{\beta}} ; \quad\left(M_{\gamma}{ }^{\delta}\right)^{\dagger}=M_{\dot{\gamma}}^{\dot{\delta}} ; \quad(Y)^{\dagger}=-Y ; \\
\left(\nabla_{a}\right)^{\dagger}=-\nabla_{a} ; \quad\left(H_{\alpha \dot{\beta}}\right)^{\dagger}=H_{\beta \dot{\alpha}} ; \quad\left(W_{\alpha \beta \gamma}\right)^{\dagger}=\bar{W}_{\dot{\alpha} \dot{\beta} \dot{\gamma}}
\end{array}
$$




\section{B Solving the Bianchi identities}

In this appendix we give some details of the solution of the Bianchi identities. There are two different Bianchi identities that we will solve: the Bianchi identity associated with the antisymmetric tensor $B_{M N}$ and the Bianchi's associated with the covariant derivatives. The first one just expresses the fact that $H_{L M N}$ is a closed 3-form (notice that the indices in $H_{L M N}$ are curved indices). The second ones follow from the Jacobi identities for the covariant derivatives.

Let us start from the latter. The equation we need to solve is the following 44

$$
\Delta_{A B C}{ }^{D}=R_{[A B C)}^{D}+w(D) F_{[A B} \delta_{C)}^{D}
$$

where

$$
\Delta_{A B C}{ }^{D}=\nabla_{[A} T_{B C)}^{D}-T_{[A B \mid}^{E} T_{[E \mid C)}^{D} .
$$

There are two other equations that follow from the Jacobi identities. One of them involves the covariant derivative of the curvatures $R_{A B C}{ }^{D}$ and the other the covariant derivative of the $U(1)$ curvature. They do not yield any further information, though, since they become true identities once (B.1]) is satisfied (Dragon's theorem [46]).

The second set of Bianchi identities is associated with the antisymmetric tensor. The field strength $H_{L M N}$ is a closed 3-form. Therefore,

$$
d H_{L M N}=0
$$

One now writes $(\overline{\mathrm{B} .3})$ in terms of covariant derivatives and with all indices flat. The result is a sum of two terms. The first ones vanish if the supergravity Bianchi's are satisfied. So, we are left with the following identity to solve

$$
\nabla_{[A} H_{B C D)}-T_{[A B \mid}^{E} H_{E \mid C D)}=0 .
$$

This equation has the same form as in flat superspace.

To solve (B.1) and (B.4) we decompose all tensors in irreducible representations of the Lorentz group and substitute these in (B.1) and (B.4). By further decomposing the left and right hand side of $(\mathbb{B} .1)$ and (B.4) into Lorentz irreducible pieces we get relations among the different Lorentz components of the curvatures and the torsions. Eventually all tensors are expressed in terms of a few superfields that satisfy certain differential relations.

The Lorentz decomposition is achieved by just symmetrizing and antisymmetrizing all similar indices. For example, the curvature $R_{\dot{\alpha} \beta \gamma \delta}$ contains an $(3 / 2,1 / 2)$ and an $(1 / 2$, 1/2) irreducible component Explicitely we have

$$
R_{\dot{\alpha} \beta \gamma \delta}=r_{\dot{\alpha}, \beta \gamma \delta}+C_{\beta(\gamma} r_{\delta) \dot{\alpha}}
$$

where $r_{\dot{\alpha}, \beta \gamma \delta}$ is symmetric in $\beta, \gamma, \delta$ and represents the $(3 / 2,1 / 2)$ component and $r_{\delta \dot{\alpha}}$ represents the $(1 / 2,1 / 2)$ piece. To give a second example, consider the torsion component $T_{\alpha b c}$. It contains the 6 different Lorentz components, namely $(3 / 2,0) \oplus(1 / 2,0) \oplus(1 / 2,0) \oplus$

\footnotetext{
${ }^{8}$ Our notation $(a, b)$ refers to the representation $(a, b)$ of the Lorentz group $S L(2, C)$, where $a$ is the spin carried by the undotted indices and $b$ is the spin carried by the dotted indices.
} 
$(3 / 2,1) \oplus(1 / 2,1) \oplus(1 / 2,1)$. The tree level constraint (3.39), however, eliminates half of them, and we get the following decomposition

$$
T_{\alpha b c}=C_{\dot{\beta} \dot{\gamma}}\left[K_{\alpha \beta \gamma}+C_{\alpha(\beta} K_{\gamma)}\right]+C_{\beta \gamma} K_{\alpha \dot{\beta} \dot{\gamma}}
$$

The Lorentz decomposition of the rest of the tensors is tabulated below

$$
\begin{aligned}
R_{\alpha \beta \gamma \delta} & =r_{\alpha \beta \gamma \delta}+\left(C_{\delta(\alpha} r_{\beta) \gamma}+\gamma \leftrightarrow \delta\right)+C_{\alpha(\gamma} C_{\delta) \beta} r \\
R_{\dot{\alpha} b \gamma \delta} & =r_{\dot{\alpha} \dot{\beta}, \beta \gamma \delta}+C_{\dot{\alpha} \dot{\beta}} r_{\beta \gamma \delta}+C_{\beta(\gamma} r_{\delta) \dot{\alpha} \dot{\beta}}+C_{\dot{\alpha} \dot{\beta}} C_{\beta(\gamma} r_{\delta)} \\
R_{\alpha b \gamma \delta} & =r_{\dot{\beta}, \alpha \beta \gamma \delta}+\left(C_{\alpha \beta} r_{\gamma \delta \dot{\beta}}+\operatorname{cyclic} \operatorname{in} \beta, \gamma, \delta\right)-C_{\beta(\gamma} r_{\delta) \alpha \dot{\beta}}^{\prime}+C_{\alpha(\gamma} C_{\delta) \beta} r_{\dot{\beta}} \\
R_{a b \gamma \delta} & =C_{\dot{\alpha} \dot{\beta}}\left[W_{\alpha \beta \gamma \delta}+\left(C_{\delta(\alpha} W_{\beta) \gamma}+\gamma \leftrightarrow \delta\right)+C_{\alpha(\gamma} C_{\delta) \beta} W\right]+C_{\alpha \beta} W_{\dot{\alpha} \dot{\beta}, \gamma \delta} \\
F_{\alpha b} & =C_{\alpha \beta} f_{\dot{\beta}}+f_{\alpha \beta, \dot{\beta}} \\
F_{a b} & =C_{\alpha \beta} f_{\dot{\alpha} \dot{\beta}}+C_{\dot{\alpha} \dot{\beta}} f_{\alpha \beta} \\
T_{a b \gamma} & =C_{\alpha \beta} W_{\gamma, \dot{\alpha} \dot{\beta}}+C_{\dot{\alpha} \dot{\beta}}\left(C_{\gamma(\alpha} W_{\beta)}+W_{\alpha \beta \gamma}\right) \\
H_{a b c} & =C_{\gamma \alpha} C_{\dot{\gamma} \dot{\beta}} H_{\dot{\alpha} \beta}-C_{\gamma \beta} C_{\dot{\gamma} \dot{\alpha}} H_{\dot{\beta} \alpha}=\frac{1}{2}\left(C_{\dot{\alpha} \dot{\beta}} C_{\gamma(\alpha \mid} H_{\dot{\gamma} \mid \beta)}-C_{\alpha \beta} C_{\dot{\gamma}(\dot{\alpha}} H_{\dot{\beta}) \gamma}\right)
\end{aligned}
$$

The Lorentz decomposition of the remaining components can be obtained from the ones listed above by hermitian conjugation, see (A.17), A.18). For example,

$$
R_{\alpha \dot{\beta} \dot{\gamma} \dot{\delta}}=\bar{r}_{\alpha, \dot{\beta} \dot{\gamma} \dot{\delta}}+C_{\dot{\beta}\left(\dot{\gamma}^{\prime}\right.} \bar{\delta}_{\dot{\delta}) \alpha}
$$

To organize the calculation we start with the Bianchi's of lowest dimension (vector indices have dimension 1 and spinor indices dimension 1/2; then "dimension = lower indices - upper indices") and work our way up. The first non-trivial equation appears at dimension 1/2 and it involves only torsions. As mentioned in Appendix A the Lorentz group acts reducibly in target space. This implies that in the equation $(\dot{\alpha}, \beta, \gamma, d)$ 向 the right hand side vanishes identically. Then the equation yields

$$
T_{(\alpha, \beta) \dot{\beta}}^{c}=0 .
$$

Using (B.6) we get that the torsion $T_{\alpha b}{ }^{c}$ vanishes.

Because the curvatures $R_{A B c d}$ are determined from the curvatures $R_{A B \gamma \delta}$ and $R_{A B \dot{\gamma} \dot{\delta}}$ it is, in general, advisable to analyze first the identities $(A, B, \tilde{\gamma}, \tilde{\delta})$. We start, however, by partly analyzing the equation $(\alpha, \dot{\beta}, c, d)$. The right hand side is equal to $R_{\alpha \dot{\beta} c d}$. The left hand side is given by

$$
\Delta_{\alpha \dot{\beta} c d}=2 i T_{\alpha \dot{\beta} c d}+i C_{\alpha \delta} C_{\dot{\beta} \dot{\delta}}\left(T_{c \epsilon}{ }^{\epsilon}+T_{c \dot{\epsilon}} \dot{\epsilon}\right)
$$

To proceed further we need a result from the Bianchi's related to the antisymmetric tensor. From the equation $(a, \dot{\beta}, \gamma, \delta)$ (where now by $(a, \dot{\beta}, \gamma, \delta)$ we mean (B.4) with $A=a, B=\dot{\beta}, C=\gamma, D=\delta$ ) we get

$$
T_{\alpha \dot{\beta}, b c}=-2 H_{\alpha \dot{\beta}, b c}+\frac{1}{2} C_{\gamma \delta} C_{\dot{\gamma} \dot{\delta}}\left(T_{\alpha \dot{\beta}, \epsilon}{ }^{\epsilon}+T_{\alpha \dot{\beta}, \dot{\epsilon}}{ }^{\dot{\epsilon}}\right)-\frac{1}{2} C_{\alpha \delta} C_{\dot{\beta} \dot{\delta}}\left(T_{c \epsilon}{ }^{\epsilon}+T_{c \dot{\epsilon}}{ }^{\dot{\epsilon}}\right)
$$

\footnotetext{
${ }^{9} \mathrm{By}(\dot{\alpha}, \beta, \gamma, d)$ we mean equation (B.1) with $A=\dot{\alpha}, B=\beta, C=\gamma, D=d$.
} 
Inserting this into (B.9) we obtain

$$
\Delta_{\alpha \dot{\beta} c d}=-4 i H_{\alpha \dot{\beta}, b c}+i C_{\gamma \delta} C_{\dot{\gamma} \dot{\delta}}\left(T_{\alpha \dot{\beta}, \epsilon}{ }^{\epsilon}+T_{\alpha \dot{\beta}, \dot{\epsilon}}{ }^{\dot{\epsilon}}\right)
$$

However, $\Delta_{\alpha \dot{\beta} c d}$ is antisymmetric in the indices $c$ and $d$ since it is equal to $R_{\alpha, \dot{\beta}, c d}$. Hence,

$$
T_{\alpha \dot{\beta}, \epsilon}{ }^{\epsilon}+T_{\alpha \dot{\beta}, \dot{\epsilon}}^{\dot{\epsilon}}=0
$$

which combined with the conventional constraint

$$
T_{\alpha \dot{\beta}, \epsilon}{ }^{\epsilon}-T_{\alpha \dot{\beta}, \dot{\epsilon}}^{\dot{\epsilon}}=0
$$

yields

$$
T_{\alpha \dot{\beta}, \epsilon}{ }^{\epsilon}=T_{\alpha \dot{\beta}, \dot{\epsilon}}^{\dot{\epsilon}}=0
$$

So, equations $(\mathbb{B . 9})$ and $(\mathbb{B . 1 0})$ become

$$
\begin{aligned}
& R_{\alpha \dot{\beta} c d}=-4 i H_{\alpha \dot{\beta}, b c} \\
& T_{a b c}+2 H_{a b c}=0 .
\end{aligned}
$$

We now proceed with the supergravity Bianchi's. We will return to the antisymmetric tensor Bianchi's after we solve completely the gravity ones. In dimension 1 the left hand side of (B.1) vanishes identically (due to constraints). There are 6 (non-trivial) equations at this level. Three of those, namely the equations $(\dot{\alpha}, \dot{\beta}, \gamma, \delta),(\dot{\alpha}, \beta, \gamma, \delta)$, and $(\alpha, \beta, \gamma, \delta)$, yield

$$
\begin{aligned}
& R_{\dot{\alpha} \dot{\beta} \gamma \delta}=r_{\dot{\alpha}, \beta \gamma \delta}=r_{\alpha \beta \gamma \delta}=0, \\
& F_{\dot{\alpha} \dot{\beta}}=0, \quad F_{\dot{\alpha} \beta}=-2 r_{\beta \dot{\alpha}}, \quad F_{\alpha \beta}=4 r_{\alpha \beta} .
\end{aligned}
$$

The other three equations are the hermitian conjugates of these ones. The results are the hermitian conjugates of the equations (B.16). (One should remember that our $U(1)$ generators are anti-hermitian; this yields an extra minus sign in the $U(1)$ sector).

At dimension $3 / 2$ we have 4 equations. The equation $(\dot{\alpha}, b, \gamma, \delta)$ yields

$$
\begin{aligned}
& r_{\dot{\alpha} \dot{\beta}, \beta \gamma \delta}=0, \quad r_{\alpha \dot{\beta} \dot{\gamma}}=i W_{\alpha \dot{\beta} \dot{\gamma}}, \quad r_{\alpha}=i W_{\alpha}, \quad r_{\alpha \beta \gamma}=-2 i W_{\alpha \beta \gamma}, \\
& \bar{f}_{\alpha}=6 i W_{\alpha}, \quad \bar{f}_{\dot{\alpha} \dot{\beta}, \gamma}=-2 i W_{\gamma \dot{\alpha} \dot{\beta}}
\end{aligned}
$$

Next we consider the equation $(\alpha, b, \gamma, \delta)$. It yields

$$
r_{\dot{\beta}, \alpha \beta \gamma \delta}=0, \quad r_{\alpha \beta \dot{\gamma}}^{\prime}=-\frac{1}{2} r_{\alpha \beta \dot{\gamma}}=\frac{1}{6} f_{\alpha \beta, \dot{\gamma}}, \quad f_{\dot{\beta}}=-2 r_{\dot{\beta}}
$$

Combining these results with their hermitian conjugates we get

$$
r_{\dot{\alpha}}=3 i \bar{W}_{\dot{\alpha}}, \quad r_{\alpha \beta \dot{\gamma}}^{\prime}=\frac{i}{3} \bar{W}_{\dot{\gamma} \alpha \beta} .
$$

At the dimension 2 , from the equation $(a, b, \gamma, \delta)$ we get

$$
\begin{aligned}
& W_{\alpha \beta \gamma \delta}=\frac{1}{24} \nabla_{(\alpha} W_{\beta \gamma \delta)}, W_{\alpha \beta}=\frac{1}{4} \nabla_{(\alpha} W_{\beta)}-\frac{1}{8} \nabla^{\delta} W_{\delta \alpha \beta}, \\
& W=\frac{1}{2} \nabla^{\delta} W_{\delta}, \quad W_{\dot{\alpha} \dot{\beta}, \gamma \delta}=\frac{1}{2} \nabla_{(\gamma} W_{\delta), \dot{\alpha} \dot{\beta}}, \\
& f_{\alpha \beta}=-\nabla^{\delta} W_{\delta \alpha \beta}-\nabla_{(\alpha} W_{\beta)}, \quad f_{\dot{\alpha} \dot{\beta}}=-\nabla^{\delta} W_{\delta \dot{\alpha} \dot{\beta}} .
\end{aligned}
$$


The hermitian conjugate of this equations implies that $f_{\alpha \beta}=-\overline{\left(f_{\dot{\alpha} \dot{\beta}}\right)}$, where the bar indicates that dotted and undotted indices should be interchanged. Explicitely, we get

$$
\nabla^{\dot{\delta}} \bar{W}_{\dot{\delta} \alpha \beta}+\nabla^{\delta} W_{\delta \alpha \beta}+\nabla_{(\alpha} W_{\beta)}=0 .
$$

We now move to the case where the equations involve curvatures with two bosonic indices as the last two indices. There is no $U(1)$ contribution in this sector because vector indices do not carry $U(1)$ charge. The results are tabulated below

$$
\begin{array}{ll}
(\alpha, \beta, c, d): & r_{\alpha \beta}=r=0 \\
(\alpha, \dot{\beta}, c, d): & r_{\alpha \dot{\beta}}=-\bar{r}_{\dot{\beta} \alpha}=-2 i H_{\dot{\beta} \alpha} \\
(\alpha, b, c, d): & \bar{W}_{\dot{\alpha}}=\frac{i}{6} \nabla^{\beta} H_{\dot{\alpha} \beta}, \bar{W}_{\dot{\alpha} \beta \gamma}=-\frac{i}{2} \nabla_{(\beta \mid} H_{\dot{\alpha} \mid \gamma)} \\
(a, b, c, d): & \bar{W}_{\dot{\alpha} \dot{\beta}}=-\frac{1}{8} \nabla_{\gamma(\dot{\alpha}} H_{\dot{\beta})}{ }^{\gamma}, W_{\alpha \beta}=\frac{1}{8} \nabla_{(\alpha \mid \dot{\gamma}} H_{\mid \beta)}, W, W=-\frac{3}{2} \nabla_{a} H^{a}, \\
& \bar{W}_{\alpha \beta, \dot{\gamma} \dot{\delta}}-W_{\dot{\gamma} \dot{\delta}, \alpha \beta}=-\frac{1}{2}\left(\nabla_{\alpha(\dot{\gamma}} H_{\dot{\delta}) \beta}+\alpha \leftrightarrow \beta\right)
\end{array}
$$

In deriving the results in (B.22) we have used (B.15).

Next we need to check the consistency between the results in (B.20) and the ones in (B.22). This yields

$$
W=\bar{W}=\frac{i}{12} \nabla^{\dot{\alpha}} \nabla^{\alpha} H_{\dot{\alpha} \alpha}, \quad \nabla_{a} H^{a}=0 .
$$

All curvatures and torsions can now be expressed in terms of the superfields $H_{\dot{\alpha} \beta}$, $W_{\alpha \beta \gamma}$ and $\bar{W}_{\dot{\alpha} \dot{\beta} \dot{\gamma}}$. The results are gathered below.

$$
\begin{aligned}
& R_{\dot{\alpha} \beta \gamma \delta}=2 i H_{\dot{\alpha}(\gamma} C_{\delta) \beta} \\
& R_{\dot{\alpha} b \gamma \delta}=-2 i C_{\dot{\alpha} \dot{\beta}} W_{\beta \gamma \delta}+\left[C_{\beta \gamma}\left(-\frac{1}{2} \nabla_{(\dot{\alpha}} H_{\dot{\beta}) \delta}+\frac{1}{6} C_{\dot{\alpha} \dot{\beta}} \nabla^{\dot{\epsilon}} H_{\dot{\epsilon} \delta}\right)+\gamma \leftrightarrow \delta\right] \\
& R_{\alpha b \gamma \delta}=-\frac{1}{3} C_{\alpha \beta} \nabla_{(\gamma \mid} H_{\dot{\beta} \mid \delta)} \\
& \left.-\left[C_{\alpha \gamma}\left(\frac{1}{3} \nabla_{(\delta \mid} H_{\dot{\beta} \mid \beta)}+\frac{1}{2} C_{\delta \beta} \nabla^{\epsilon} H_{\dot{\beta} \epsilon}\right)+\frac{1}{6} C_{\beta \gamma} \nabla_{(\delta \mid} H_{\dot{\beta} \mid \alpha)}\right)+\gamma \leftrightarrow \delta\right] \\
& =\nabla_{\beta} H_{\dot{\beta}(\gamma} C_{\delta) \alpha} \\
& R_{a b \gamma \delta}=\frac{1}{24} C_{\dot{\alpha} \dot{\beta}} \nabla_{(\alpha} W_{\beta \gamma \delta)} \\
& +\left[C_{\dot{\alpha} \dot{\beta}}\left(\frac{1}{8}\left(C_{\delta \alpha} \nabla_{(\beta \mid \dot{\epsilon}} H^{\dot{\epsilon}} \mid \gamma\right)+\alpha \leftrightarrow \beta\right)+\frac{i}{12} C_{\alpha \gamma} C_{\delta \beta} \nabla^{\dot{\epsilon}} \nabla^{\epsilon} H_{\dot{\epsilon} \epsilon}\right) \\
& \left.+\frac{i}{4} C_{\alpha \beta} \nabla_{\gamma} \nabla_{(\dot{\alpha}} H_{\dot{\beta}) \delta}+\gamma \leftrightarrow \delta\right] \\
& F_{\alpha b}=C_{\alpha \beta} \nabla^{\gamma} H_{\dot{\beta} \gamma}+\nabla_{(\alpha \mid} H_{\dot{\beta} \mid \beta)}=2 \nabla_{\beta} H_{\dot{\beta} \alpha} \\
& F_{a b}=-\frac{i}{2}\left[C_{\alpha \beta} \nabla^{\delta} \nabla_{(\dot{\alpha}} H_{\dot{\beta}) \delta}+C_{\dot{\alpha} \dot{\beta}} \nabla^{\dot{\delta}} \nabla_{(\alpha \mid} H_{\dot{\delta} \mid \beta)}\right] \\
& F_{\alpha \dot{\beta}}=4 i H_{\dot{\beta} \alpha}, \\
& T_{a b \gamma}=\frac{i}{2} C_{\alpha \beta} \nabla_{(\dot{\alpha}} H_{\dot{\beta}) \gamma}+C_{\dot{\alpha} \dot{\beta}}\left[-\frac{i}{6} C_{\gamma(\alpha \mid} \nabla^{\dot{\epsilon}} H_{\dot{\epsilon} \mid \beta)}+W_{\alpha \beta \gamma}\right]
\end{aligned}
$$


Furthermore, equation (B.21) becomes

$$
\nabla^{\gamma} W_{\gamma \alpha \beta}=\frac{i}{6} \nabla_{(\alpha \mid} \nabla^{\dot{\gamma}} H_{\dot{\gamma} \mid \beta)}+\frac{i}{2} \nabla^{\dot{\gamma}} \nabla_{(\alpha \mid} H_{\dot{\gamma} \mid \beta)}
$$

The last non-trivial equation is the equation $(a, b, \gamma, \dot{\delta})$. It yields

$$
\nabla_{\dot{\delta}} T_{a b \gamma}=0
$$

This, in turn, implies that $W_{\alpha \beta \gamma}$ is a chiral superfield and $H_{a}$ is a linear superfield

$$
\nabla_{\dot{\delta}} W_{\alpha \beta \gamma}=0 ; \quad \nabla^{\beta} \nabla_{\beta} H_{a}=0 .
$$

The resulting supergravity algebra is given in (3.54)-(3.57). This supergravity algebra is actually invariant under local superscale and local $U(1)$ transformations as we now describe. To discover these transformations we start from the transformation rule of the vierbein

$$
\delta E_{\alpha}{ }^{M}=\frac{1}{2} L E_{\alpha}{ }^{M}
$$

where $L$ is a complex parameter. If $L$ is real we are dealing with local scale transormations, whereas if it is imaginary with $U(1)$ transformations. The tranformation rules of the connections are determined by requiring invariance of the supergravity algebra under local superscale and/or local $U(1)$ transformations. The manipulations involved are very similar to the ones we performed in section 5. The resulting transformation rules are given below

$$
\begin{aligned}
& \delta \nabla_{\alpha}=\frac{1}{2} L \nabla_{\alpha}+\nabla_{\beta}(L+\bar{L}) M_{\alpha}{ }^{\beta}-\left(2 \nabla_{\alpha} L+\nabla_{\alpha} \bar{L}\right) Y \\
& \delta \nabla_{\dot{\alpha}}=\frac{1}{2} \bar{L} \nabla_{\dot{\alpha}}+\nabla_{\dot{\beta}}(L+\bar{L}) M_{\dot{\alpha}}{ }^{\dot{\beta}}+\left(2 \nabla_{\dot{\alpha}} \bar{L}+\nabla_{\dot{\alpha}} L\right) Y \\
& \delta \nabla_{a}=\frac{1}{2}(L+\bar{L}) \nabla_{a}+\frac{i}{2} \nabla_{\dot{\alpha}}(L+\bar{L}) \nabla_{\alpha}+\frac{i}{2} \nabla_{\alpha}(L+\bar{L}) \nabla_{\dot{\alpha}} \\
& +\frac{i}{2} \nabla_{\beta} \nabla_{\dot{\alpha}}(L+\bar{L}) M_{\alpha}{ }^{\beta}+\frac{i}{2} \nabla_{\dot{\alpha}} \nabla_{\beta}(L+\bar{L}) M_{\alpha}{ }^{\beta}+\frac{i}{2}\left(\nabla_{\alpha} \nabla_{\dot{\alpha}} \bar{L}-\nabla_{\dot{\alpha}} \nabla_{\alpha} L\right) Y
\end{aligned}
$$

In addition

$$
\begin{aligned}
& \delta H_{a}=\frac{1}{2}(L+\bar{L}) H_{a}-\frac{i}{4}\left[\nabla_{\alpha}, \nabla_{\dot{\alpha}}\right](L+\bar{L}) \\
& \delta W_{\alpha \beta \gamma}=\frac{1}{2}(2 \bar{L}+L) W_{\alpha \beta \gamma} .
\end{aligned}
$$

The parameter $L$ satisfies the equation

$$
\nabla^{2}(L+\bar{L})=0
$$

This means $L$ is an imaginary unconstrained superfield for $U(1)$ transformations and a real linear superfield for scale transformations. From these transformation rules we can read off the relation between the conformal weight $d$ and the $U(1)$ charge $w$ of a 
chiral superfield, using the fact that scale transformations should respect the chirality condition, $\delta\left(\nabla_{\dot{\alpha}} \phi\right)=0$. This yields

$$
d+3 w=0
$$

We now return to the antisymmetric tensor Bianchi's. Having already solved the supergravity Bianchi's it is rather easy to solve (B.4). Most of the components of $H_{A B C}$ have already been determined by the tree-level constraints

$$
H_{a \tilde{\beta} \tilde{\gamma}}=H_{a b \tilde{\gamma}}=0 ; \quad H_{\alpha \dot{\beta} c}=-i C_{\alpha \gamma} C_{\dot{\beta} \dot{\gamma}}
$$

Equations $(\alpha, \beta, \gamma, \delta),(\dot{\alpha}, \beta, \gamma, \delta),(\dot{\alpha}, \dot{\beta}, \gamma, \delta)$ and $(a, b, \gamma, \delta)$ yield

$$
\begin{aligned}
& \nabla_{\alpha} H_{\beta \gamma \delta}+\text { cyclic in } \alpha, \beta, \gamma, \delta=0, \\
& \nabla_{\dot{\alpha}} H_{\beta \gamma \delta}+\nabla_{\beta} H_{\dot{\alpha} \gamma \delta}+\nabla_{\gamma} H_{\dot{\alpha} \beta \delta}+\nabla_{\delta} H_{\dot{\alpha} \beta \gamma}=0, \\
& \nabla_{\dot{\alpha}} H_{\dot{\beta} \gamma \delta}+\nabla_{\dot{\beta}} H_{\dot{\alpha} \gamma \delta}+\nabla_{\gamma} H_{\dot{\alpha} \dot{\beta} \delta}+\nabla_{\delta} H_{\dot{\alpha} \dot{\beta} \gamma}=0, \\
& T_{a b} \tilde{\epsilon}_{\tilde{\epsilon} \gamma \delta}=0 .
\end{aligned}
$$

We conclude that $H_{\alpha \beta \gamma}=H_{\dot{\alpha} \beta \gamma}=H_{\dot{\alpha} \dot{\beta} \gamma}=0$.

\section{References}

[1] N. Berkovits, Nucl. Phys. B395 (1993) 77.

[2] N. Berkovits, Nucl. Phys. B420 (1994) 332.

[3] N. Berkovits, Nucl. Phys. B431 (1994) 258.

[4] N. Berkovits and W. Siegel, Nucl. Phys. B462 (1996) 213, hep-th/9510106.

[5] W. Siegel, Phys. Lett. 211B (1988) 55.

[6] W. Siegel, Phys. Rev. D53 (1996) 3336, hep-th/9510150.

[7] N. Berkovits, A New Description of the Superstring, IFUSP-P-1212, hep-th/9604123.

[8] N. Berkovits and C. Vafa, Mod. Phys. Lett. A9 (1994) 653.

[9] N. Ohta and J.L. Petersen, Phys. Lett. B325 (1994) 67.

[10] E.S. Fradkin and A.A. Tseytlin, Phys. Lett. B158 (1985) 316; Nucl. Phys. B261 (1985) 1.

[11] R. Brooks, F. Muhammed and S.J.Gates, Jr., Nucl. Phys. B268 (1986).

[12] M.B. Green, J.H. Schwarz and E. Witten, Superstring Theory, vol. 1 (Cambridge University Press, 1987).

[13] J. Shapiro, Phys. Rev. D11 (1975) 2937; M. Ademollo, A. D'Adda, R. D'Auria, F. Gliozzi, E. Napolitano, S. Sciuto and P. Di Vecchia, Nucl. Phys. B94 (1975) 221. 
[14] J. Distler and P. Nelson, Commun. Math. Phys. 138 (1991) 273; Nucl. Phys. b366 (1991) 255.

[15] O. Bergman and B. Zwiebach, The dilaton theorem and closed string backgrounds, MIT-CTP-2376, hep-th/9411047; A. Belopolsky and B. Zwiebach, Who changes the string coupling?, MIT-CTP-2450, hep-th/9511077.

[16] T. Banks, D. Nemeschansky and A. Sen, Nucl. Phys. B277 (1986) 67.

[17] S. Gates, On Aspects and Implications of the New Covariant 4D, N=1 GreenSchwarz o-model Action, UMDEPP 96-46, hep-th/9606107.

[18] C.G. Callan, D. Friedan, E.J. Martinec and M.J. Perry, Nucl. Phys. B262 (1985) 593.

[19] A. Sen, Phys. Rev. D32 (1985) 2102.

[20] L. Alvarez-Gaumé, D. Z. Freedman and S. Mukhi, Ann. of Phy. 134 (1981) 85.

[21] S. Mukhi, Nucl. Phys. B264 (1986) 640.

[22] J. Attick, A. Dhar, Nucl. Phys. B284 (1987) 131.

[23] J. de Boer, M.B. Halpern, Unified Einstein-Virasoro Master Equation in the General Nonlinear Sigma Model, hep-th/9606025, to appear in Int. J. Mod. Phys. A.

[24] S. Gates, P. Majumdar, R. Oerter and E. Van de Ven, Phys. Lett. B214 (1988) 26.

[25] D.Z. Freedman, K. Johnson, L.I. Latorre, Nucl. Phys. B371 (1992) 329.

[26] B. de Wit, M.T. Grisaru, P. van Nieuwenhuizen, Nucl. Phys. B408 (1993) 299.

[27] G. Källén and J. Toll, J. Math. Phys. 6 (1965) 299.

[28] A.M. Polyakov, Gauge Fields and Strings, Harwood, New York, 1987; M.T. Grisaru and Peter van Nieuwenhuizen, Int. J. Mod. Phys. A7 (1992) 5891; K.A. Meisner and J. Pavelchik, Mod. Phys. Lett. A5 (1990) 763.

[29] A. Sevrin, R. Siebelink, W. Troost, Nucl. Phys. B413 (1994) 185.

[30] J. de Boer and K. Skenderis, in preparation.

[31] G. Moore and P. Nelson, Commun. Math. Phys. 100 (1985) 83.

[32] M. Green and J.H. Schwarz, Phys. Lett. B149 (1984) 117.

[33] C.M. Hull and E. Witten, Phys. Lett. B160 (1985) 398.

[34] C.M. Hull and P.K. Townsend, Phys. Lett. B178 (1986) 187.

[35] A.M. Polyakov, in Les Houches 1988, Fields, Strings and Critical Phenomena, eds. E. Brézin and J. Zinn-Justin, North-Holland, 1990.

[36] S.J. Gates, Jr. and H. Nishino, Int. J. Mod. Phys. A8 (1993) 3371. 
[37] A.A. Tseytlin, Int. J. Mod. Phys. A4 (1989) 1257.

[38] M. T. Grisaru, H. Nishino and D. Zanon, Phys. Lett. 206B (1988) 625; Nucl. Phys. B314 (1989) 363.

[39] S. Gates, P. Majumdar, N. Oerter and A. van de Ven, Nucl. Phys. B319 (1989) 291.

[40] T. Itoh and M. Takao, Int. J. Mod. Phys. A5 (1990) 2265;

G. Aldazabal and J.M. Maldacena, Int. J. Mod. Phys. A8 (1993) 3359;

M. Wehlau, Phys.Rev. D51 (1995) 1831;

N.D. Lambert, Nucl. Phys. B469 (1996) 68.

[41] G. Girardi, R. Grimm, M. Müller and J. Wess, Phys. Lett. 147B (1984) 81;

W. Lang, J. Louis and B. Ovrut, Phys. Lett. 158B (1985) 40.

[42] W. Siegel, Class. Quant. Grav. 3 (1986) L47.

[43] J. Wess and B. Zumino, Phys. Lett. 74B, (1978) 51.

[44] S. J. Gates, M. T. Grisaru, M. Roček, W. Siegel, Superspace or One Thousand and One Lessons in Supersymmetry, Benjamin/Cummings, Reading, 1983.

[45] S. Cecotti, S. Ferrara and M. Villasante, Int. J. Mod. Phys. A2 (1987) 1839;

J.-P. Derendinger, F. Quevedo and M. Quirós, Nucl. Phys. B428 (1994) 282.

[46] N. Dragon, Z. Phys. C2, (1979) 62.

[47] D. Kutasov and E. Martinec, New Principles for String/Membrane Unification, EFI96-04, hep-th/9602049;

D. Kutasov, E. Martinec and M. O'Loughlin, Vacua of $M$ Theory and $N=2$ Strings, EFI-96-07, hep-th/9603116;

E. Martinec, Geometrical Structure of M Theory, EFI-96-29, hep-th/9608017. 